\title{
57. DSDP LEG 42A BIOSTRATIGRAPHIC RANGE CHARTS
}

\author{
Germaine Bizon, Bureau d'Etudes Industrielles et de Cooperation de l'Institut Français du Pétrole, Rueil- \\ Malmaison, France \\ Maria Bianca Cita, Instituto di Geologia, University of Milano, Milano, Italy \\ Ramil Wright ${ }^{1}$, Beloit College, Department of Geology, Beloit, Wisconsin \\ and \\ Carla Müller, Geologisch-Palaeontologisches, Institut der Universität, Frankfurt Am Main, Germany
}

\section{INTRODUCTION}

Of the eight sites drilled on Leg $42 \mathrm{~A}$, all penetrated Pleistocene sediments, all except Site 377 penetrated Pliocene sediments, and all but Holes 373A and 377 penetrated the Mediterranean Evaporite Formation. However, this formation could be dated paleontologically only at Sites 372,375 , and 376 , and no real age determination could be given for evaporites at Sites 371,374 , and 378 .

Miocene sediments were recovered at Sites 372, 375, and 377 and penetration was as far as lower Burdigalian at Sites 372 and 375.

Range charts are provided here for planktonic and benthonic foraminifers but not for nannofossils, as these appear elsewhere (Müller, this volume). Also displayed is other information gleaned by the shipboard paleontologists both on other fossil groups and on minerals recorded in the sand-size fraction of their sieved samples greater than 63 microns.

\section{CONTENTS}

\section{Range Charts By M. B. Cita}

( 1) Plio-Pleistocene: Site $371 ; 37^{\circ} 36^{\prime} \mathrm{N}, 05^{\circ} 14^{\prime} \mathrm{E}$; water depth $2750 \mathrm{~m}$.

( 2) Plio-Pleistocene: Site $372 ; 40^{\circ} 01.90^{\prime} \mathrm{N}$, $04^{\circ} 47.79^{\prime} \mathrm{E}$; water depth $2695 \mathrm{~m}$.

( 3) Early and middle Miocene: Site 372 (below Core 13).

( 4) Plio-Pleistocene: Hole $373 \mathrm{~A} ; 39^{\circ} 44^{\prime} \mathrm{N}$, $13^{\circ} 00^{\prime} \mathrm{E}$; water depth $3650 \mathrm{~m}$.

( 5) Plio-Pleistocene: Site $374 ; 35^{\circ} 50.87^{\prime} \mathrm{N}$, $18^{\circ} 11.78^{\prime} \mathrm{E}$; water depth $4078 \mathrm{~m}$.

( 6) Plio-Pleistocene: Site $376 ; 34^{\circ} 52.32^{\prime} \mathrm{N}$, $31^{\circ} 48.45^{\prime} \mathrm{E}$; water depth $2101 \mathrm{~m}$.

( 7) Early and middle Miocene: Site 375 (below Core 7); $34^{\circ} 45.74^{\prime} \mathrm{N}, 31^{\circ} 45.58^{\prime} \mathrm{E}$; water depth $1900 \mathrm{~m}$.

( 8 ) Complete Section: Site $377 ; 35^{\circ} 09.25^{\prime} \mathrm{N}$, $21^{\circ} 25.86^{\prime} \mathrm{E}$; water depth $3718 \mathrm{~m}$.

( 9) Plio-Pleistocene: Hole $378 ; 35^{\circ} 56.67^{\prime} \mathrm{N}$, $25^{\circ} 06.87^{\prime} \mathrm{E}$; water depth $1835 \mathrm{~m}$.

\footnotetext{
${ }^{1}$ Present address: Department of Geology, Florida State University, Tallahassee, Florida.
}

(10) Plio-Pleistocene: Hole $378 \mathrm{~A} ; 35^{\circ} 55.67^{\prime} \mathrm{N}$, $25^{\circ} 06.97^{\prime} \mathrm{E}$, water depth $1835 \mathrm{~m}$.

Additional range-charts by $\mathrm{M}$. B. Cita are provided in Cita et al., (this volume), for Site 372, Cores 4 to 13, and for Site 375, Cores 4 to 7 .

\section{Range Charts By G. Bizon: All Sites Except 373A And 377}

(11) Site 371, Cores 1 to 8

(12) Site 372, Cores 1 to 6

(13) Site 372, Cores 9 to 15

(14) Site 372 , Cores 16 to 23

(15) Site 372 , Cores 24 to 32

(16) Site 372 , Cores 33 to 46

(17) Site 374, Cores 1 to 7

(18) Site 374 , Cores 8 to 15

(19) Site 375, Cores 1 to 7

(20) Site 375 , Cores 8 to 13

(21) Site 376, Cores 1 to 5

(22) Site 376, Cores 6 to 15

(23) Hole 378, Cores 1 to 7

(24) Hole 378A, Cores 1 to 4

\section{Range Charts By R. Wright: All Sites}

Note: The distributional data shown in these tables are not based on the study of equal sized samples nor on samples which were equally spaced stratigraphically. Samples from Sites 372, 374, and 376 were studied in much greater detail than those of the other sites; samples from the Messinian Stage and the stratigraphic intervals just above and below it were examined more closely than those of other intervals. The information in the tables reflects this bias. The greatest number of species is shown to be present in samples from these above mentioned sites and intervals, but this is probably a consequence only of their intense examination.

Although 79 of the 211 samples were examined quantitatively, the only information shown on the charts is qualitative-the presence of taxa in a sample.

The biostratigraphic zonation used in these charts is based on the studies of planktonic foraminifers and calcareous nannoplankton by the other shipboard paleontologists. Where major differences in zonation occur, e.g., Site 372, Core 9, the different interpretations are shown on the charts.

Instead of dividing the Pliocene into the standard stages: Zanclean (Tabianian) and Piacenzian, the 
planktonic foraminiferal biozonation of Cita (1975) is used here to provide further precision. Standard stage names are used for the Miocene strata.

Taxonomic notes and illustrations of the most abundant species are given in Wright (this volume).

The charts are:

(25) Site 371

(26) Site 372

(27) Hole 373A

(28) Site 374

(29) Site 375

(30) Site 376

(31) Site 377

(32) Holes 378/378A

\section{Range-Charts By C. Müller (nannofossils)}

Range charts for all sites, except Hole 373A and Site 377, appear in Müller, this volume.

\section{REFERENCES}

Cita, M. B., 1975. Planktonic foraminiferal biozonation of the Mediterranean Pliocene deep sea record. A revision: Riv. Ital. Paleont., v. 81 , no. 4 , p. 527-544.

Bizon, G. and Bizon, J.-J., 1972. Atlas des principaux foraminifères planctoniques du bassin méditerranéen, oligocène à quaternaire. Technip. ed., p. 1-316.

Blow, W. H., 1969. Late middle Eocene to Recent planktonic foraminiferal biostratigraphy. Brill ed., p. 199-421. 
TABLE 1

Occurrence Ranges of Plio-Pleistocene Planktonic Foraminifers and Other Fossils at Site 371

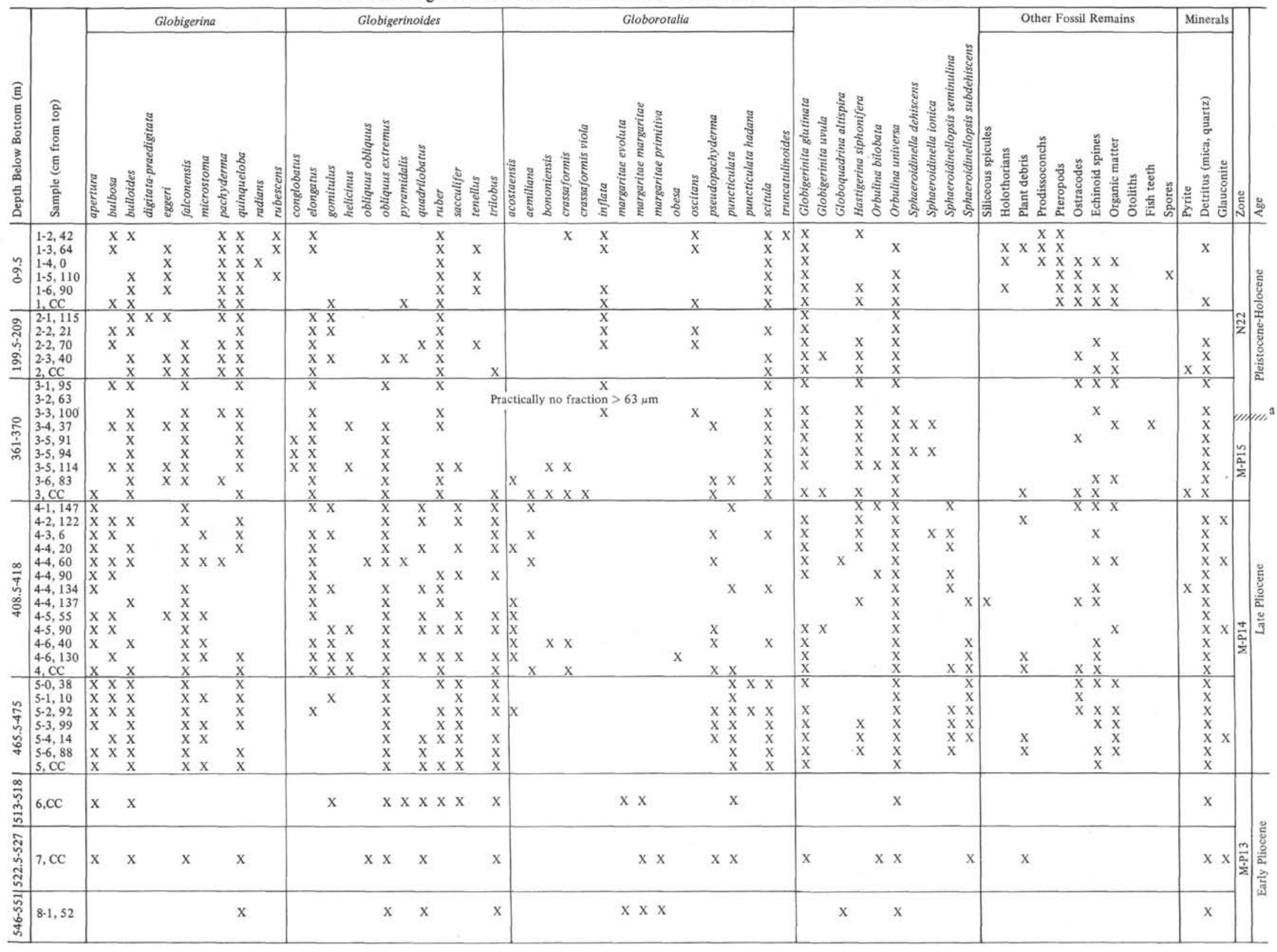


TABLE 2

Occurrence Ranges of Plio-Pleistocene Planktonic Foraminifers and Other Fossils at Site 272

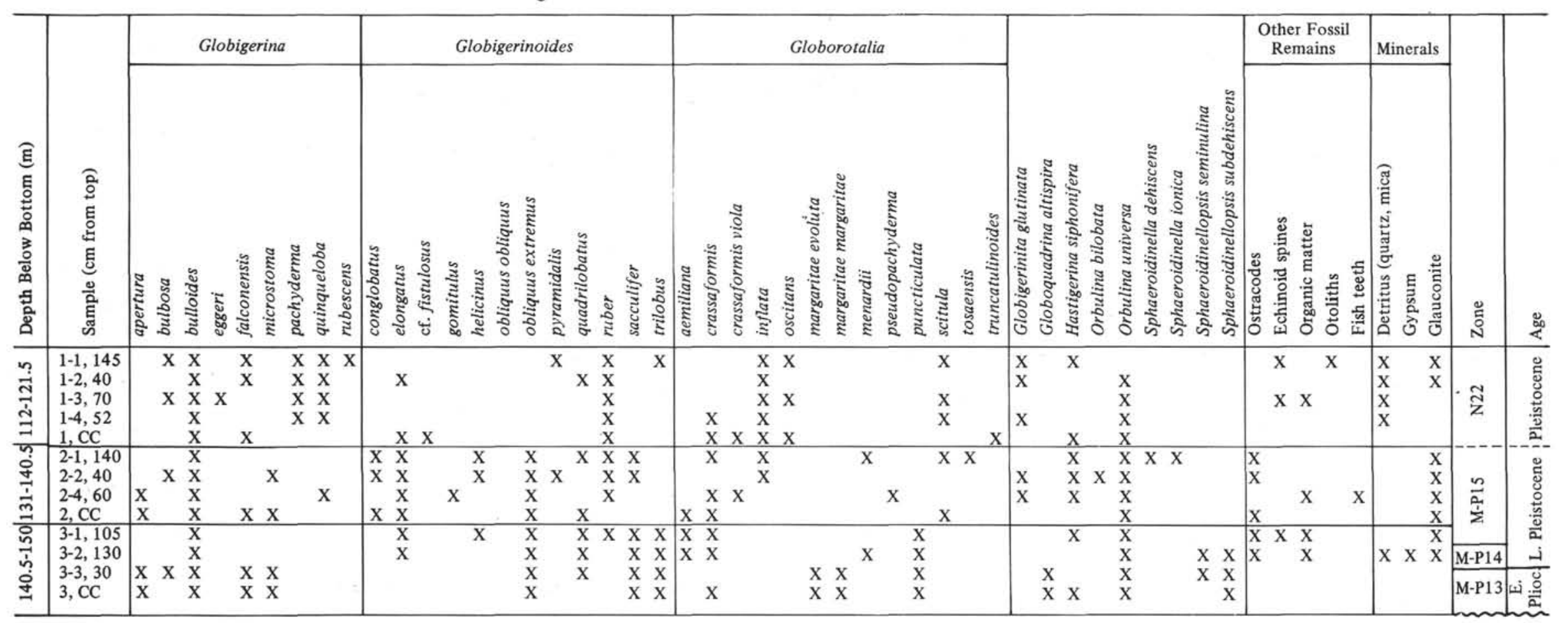


TABLE 3

Occurrence Ranges of Early and Middle Miocene Planktonic Foraminifers at Site 372

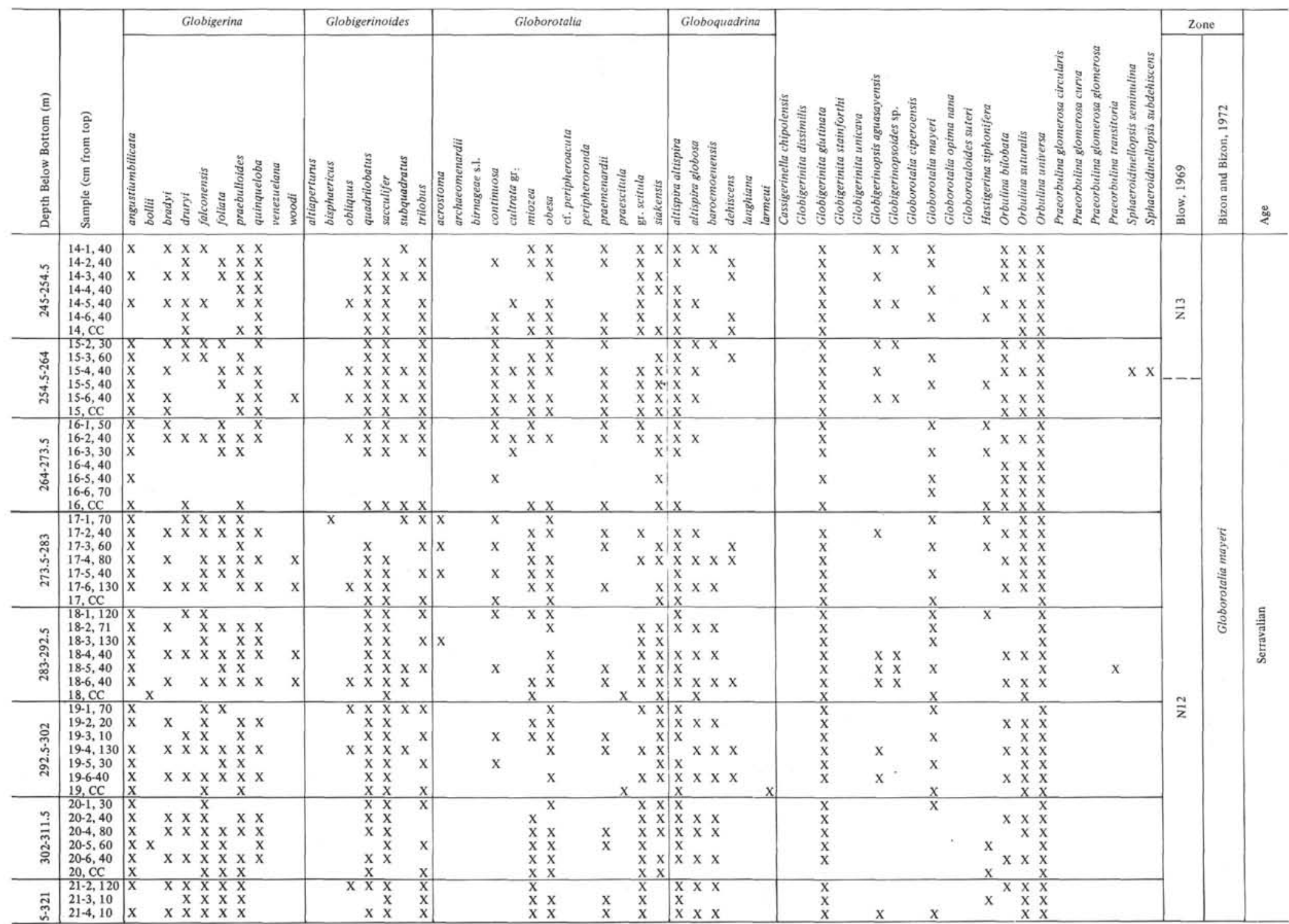




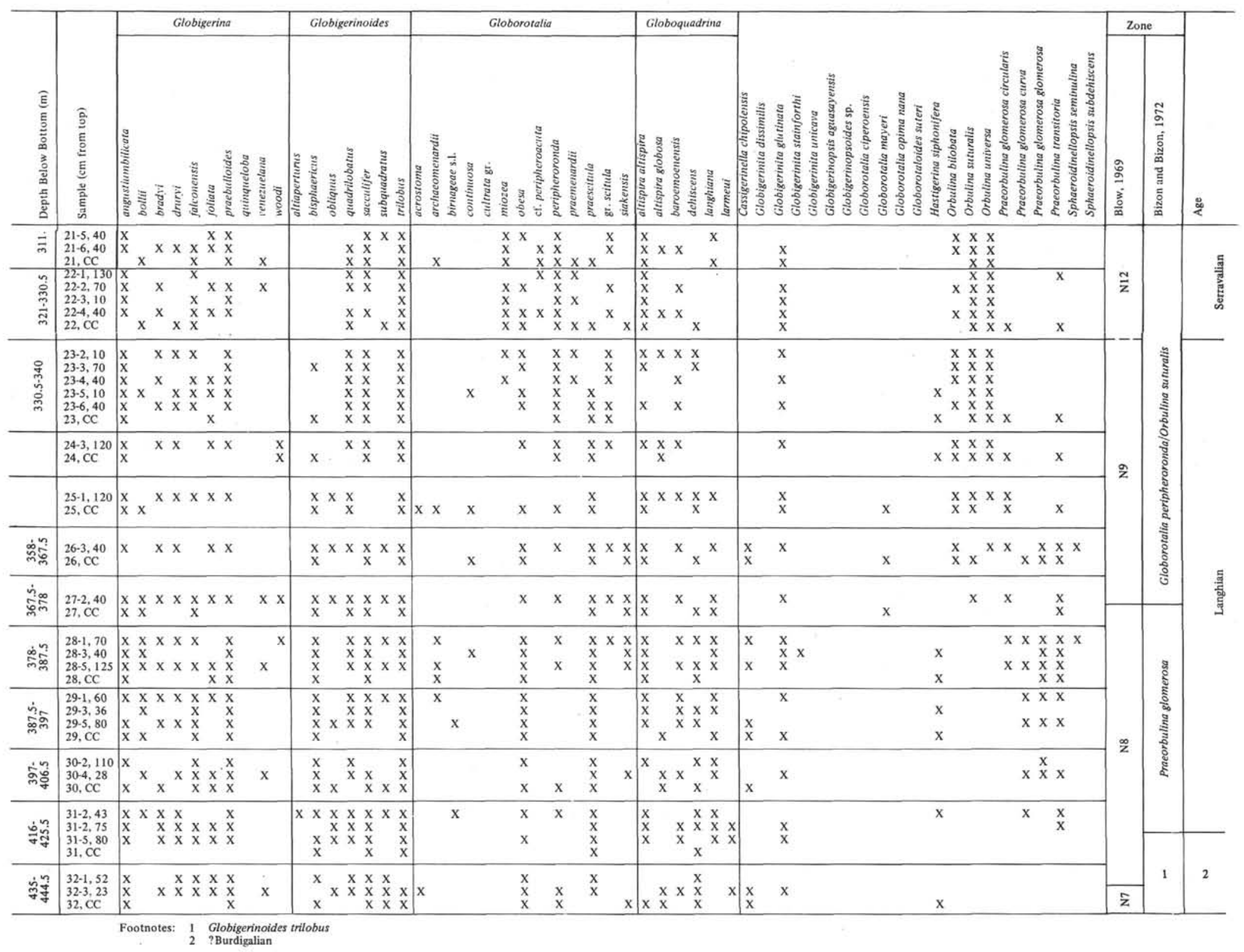


TABLE 3 - Continued

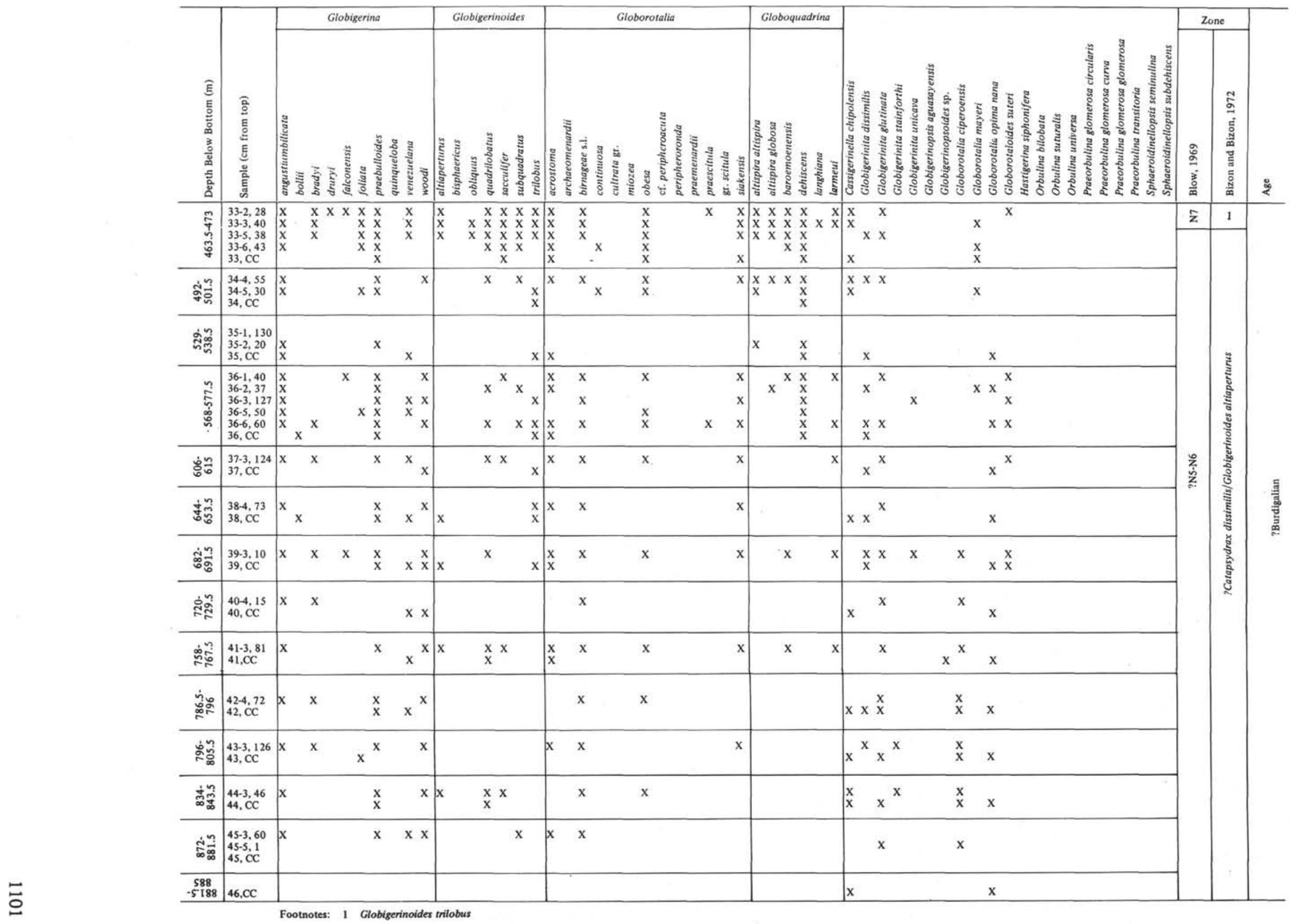


TABLE 4

Occurrence Ranges of Plio-Pliestocene Planktonic Foraminifers and Other Fossils at Site 373A

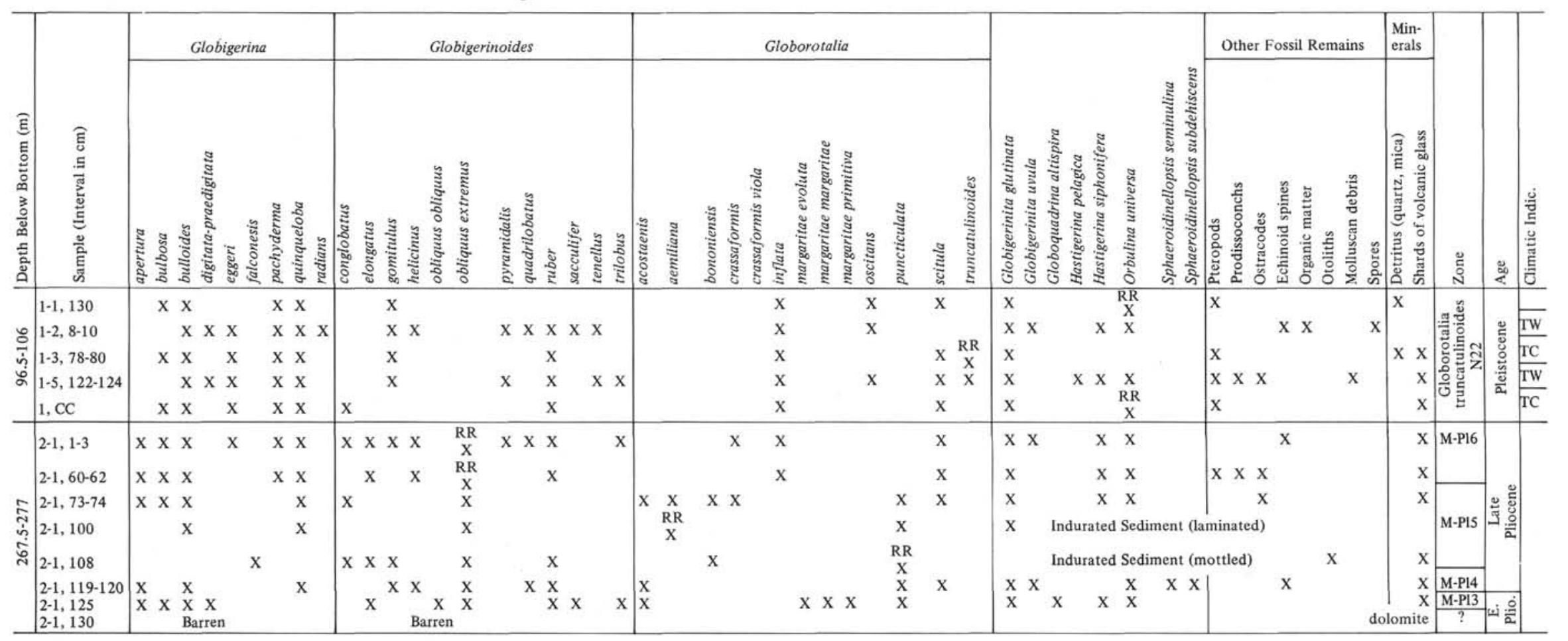


TABLE 5

Occurrence Ranges of Plio-Pleistocene Planktonic Foraminifers at Site 374

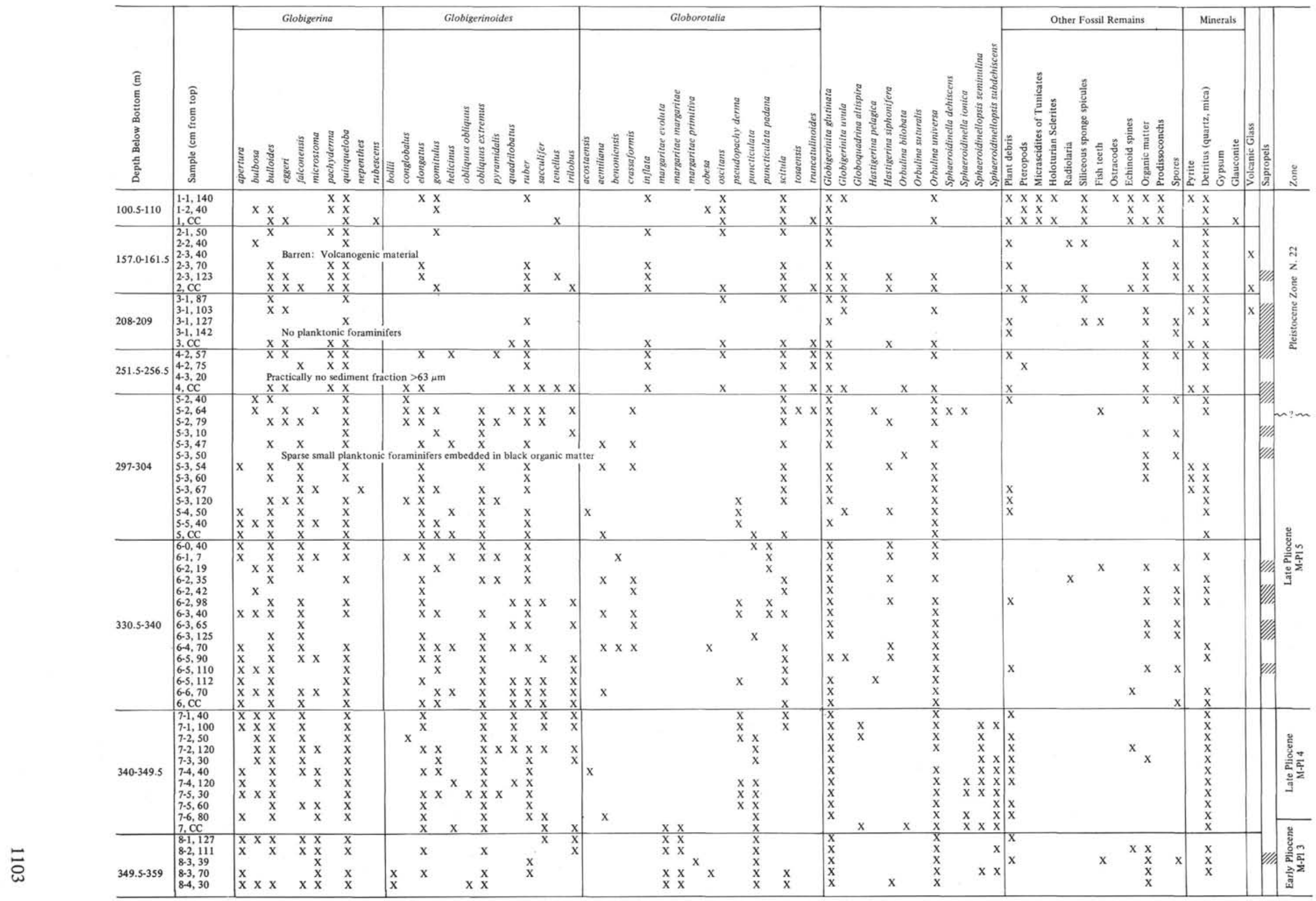

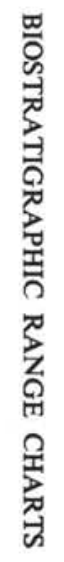


TABLE 5 - Continued

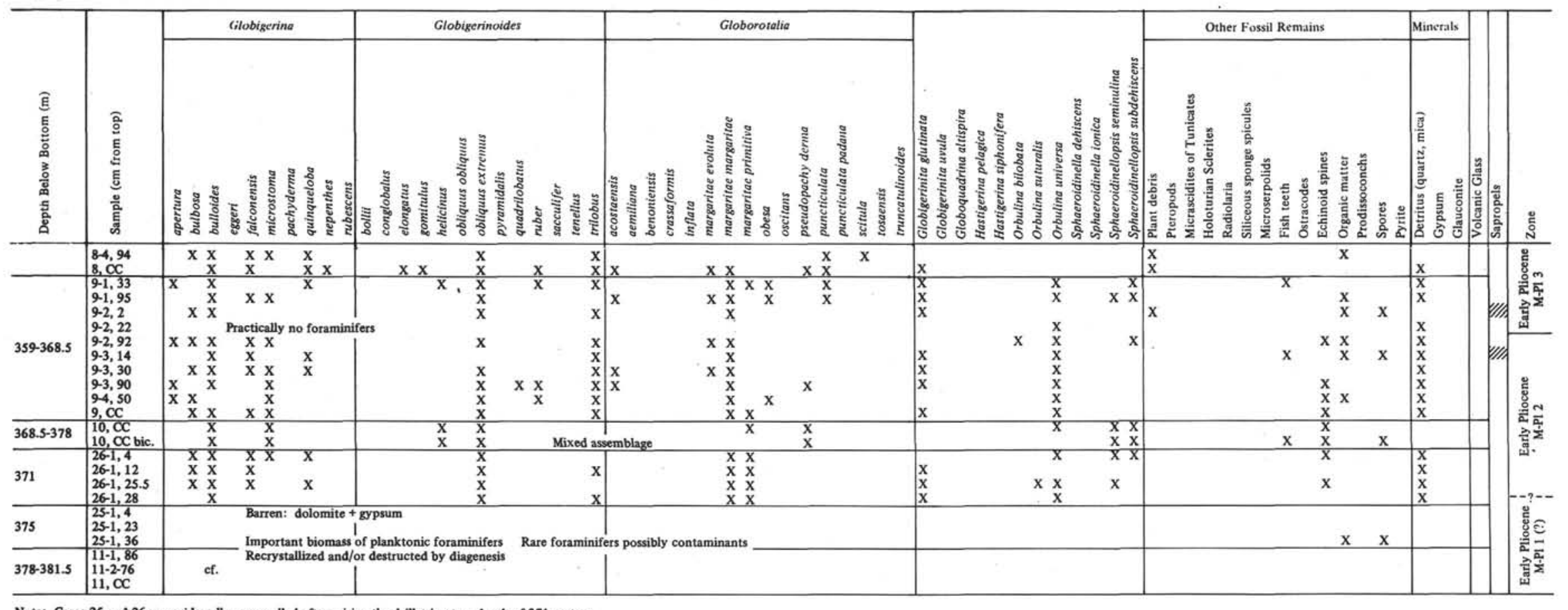

Note: Cores 25 and 26 were sidewall cores, pulled after raising the drillstring to a depth of 371 meters 
TABLE 6

Occurrence Ranges of Plio-Pleistocene Planktonic Foraminifers and Other Fossils at Site 376

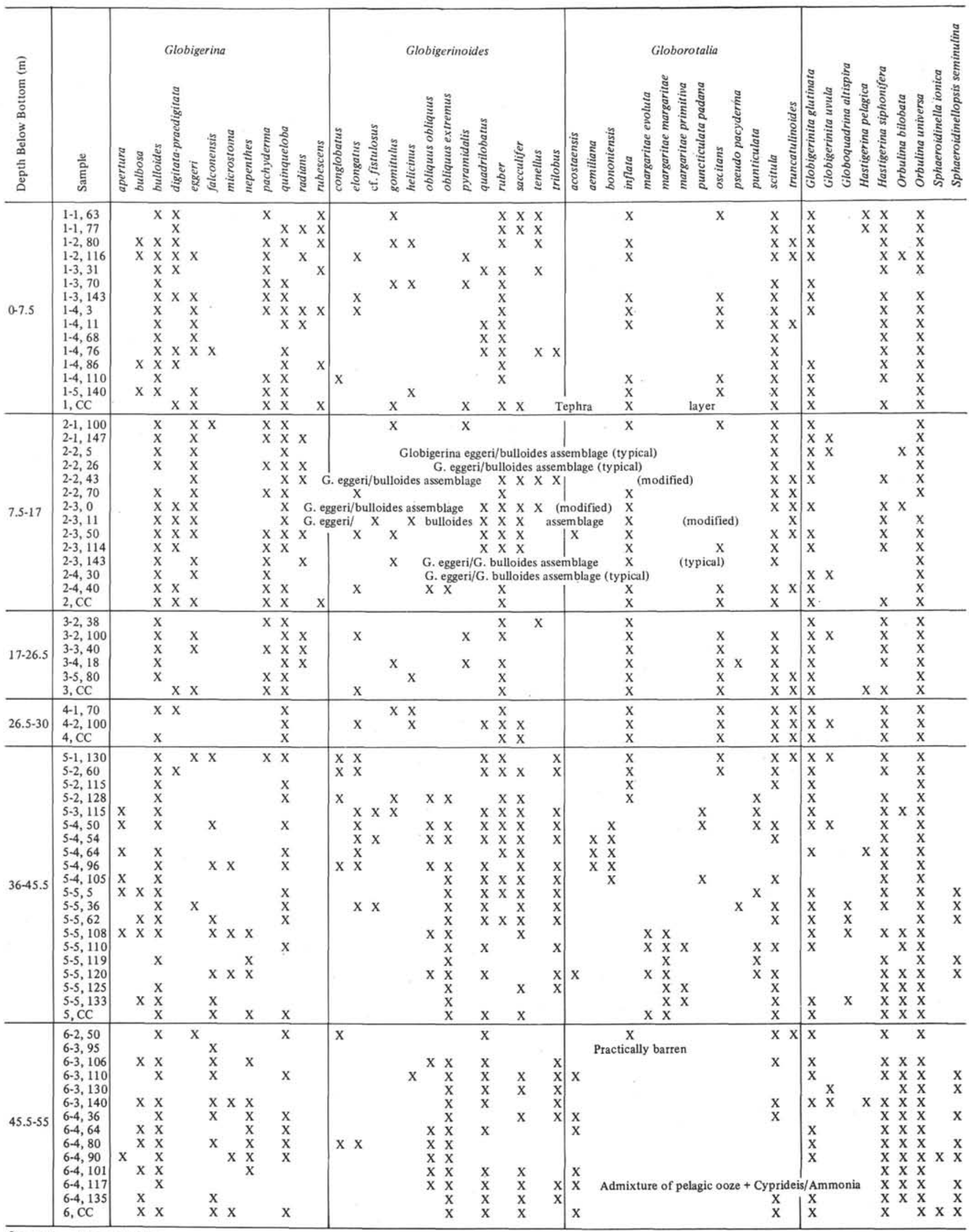

${ }^{\mathrm{a}}$ Probable artificial repetition. 
TABLE 6 - Continued

\begin{tabular}{|c|c|c|c|c|c|c|c|c|c|c|c|c|c|}
\hline 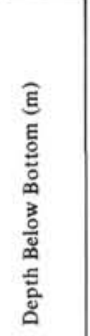 & 总 & 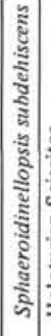 & 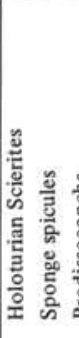 & 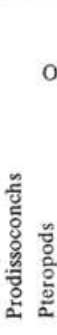 & 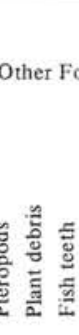 & के & 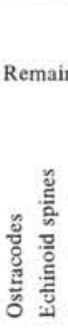 & 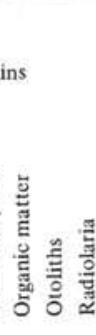 & 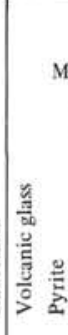 & 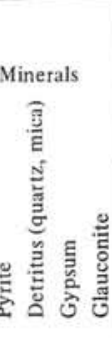 & 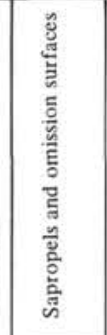 & 总 & స్ \\
\hline $0-7.5$ & $\begin{array}{l}1-1,63 \\
1-1,77 \\
1-2,80 \\
1-2,116 \\
1-3,31 \\
1-3,70 \\
1-3,143 \\
1-4,3 \\
1-4,11 \\
1-4,68 \\
1-4,76 \\
1-4,86 \\
1-4,110 \\
1-5,140 \\
1, \mathrm{CC} \\
\end{array}$ & & 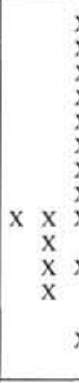 & $\begin{array}{ll}\mathrm{X} & \mathrm{x} \\
\mathrm{X} & \mathrm{X} \\
\mathrm{X} & \mathrm{X} \\
\mathrm{X} & \mathrm{X} \\
\mathrm{X} & \mathrm{x} \\
\mathrm{X} & \\
\mathrm{X} & \mathrm{X} \\
\mathrm{X} & \mathrm{X} \\
\mathrm{X} & \mathrm{x} \\
\mathrm{X} & \mathrm{X} \\
\mathrm{x} & \mathrm{X} \\
& \mathrm{X} \\
\mathrm{x} & \mathrm{X} \\
& \mathrm{X}\end{array}$ & $\begin{array}{l}x \\
x \\
x \\
x \\
x \\
x \\
x \\
x \\
x \\
x \\
x \\
x \\
x \\
x \\
x \\
x \\
x\end{array}$ & $\mathrm{x}$ & $\begin{array}{ll}x & x \\
& \\
& \\
x & x \\
& x \\
& x \\
& \\
& \\
x & x \\
x & \\
x & x \\
x & x\end{array}$ & $\begin{array}{lll}X & x & \\
X & \\
X & \\
X & \\
& \\
X & \\
X & \\
X & \\
X & x \\
X & x \\
X & \\
& \\
& \end{array}$ & $\begin{array}{ll}\mathrm{x} & \mathrm{x} \\
\mathrm{x} \\
\mathrm{x}\end{array}$ & $\begin{array}{ll}\mathrm{x} & \\
\mathrm{x} & \\
& \\
& \\
\mathrm{x} & \mathrm{x} \\
\mathrm{X} & \\
\mathrm{X} & \\
\mathrm{X} & \end{array}$ & צ'וгел & \multirow{4}{*}{$\begin{array}{l}\frac{0}{8} \\
\frac{0}{2} \\
\frac{\pi}{2}\end{array}$} & \\
\hline $7.5-17$ & $\begin{array}{l}2-1,100 \\
2-1,147 \\
2-2,5 \\
2-2,26 \\
2-2,43 \\
2-2,70 \\
2-3,0 \\
2-3,11 \\
2-3,50 \\
2-3,114 \\
2-3,143 \\
2-4,30 \\
2-4,40 \\
2, \text { CC }\end{array}$ & & & 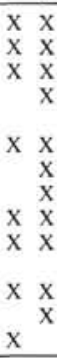 & $\begin{array}{ll}x & \\
x \\
x \\
x \\
x \\
x \\
x \\
x \\
x \\
x \\
x \\
x \\
x \\
x \\
x \\
x \\
\\
x\end{array}$ & $\begin{array}{l}x \\
x \\
x\end{array}$ & $\begin{array}{ll}x & x \\
& x \\
& \\
& \\
x & \\
& x \\
x & x \\
x & x \\
& x \\
& x \\
x & x \\
& x \\
\end{array}$ & 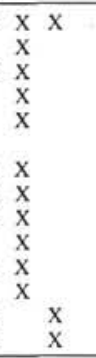 & $\begin{array}{ll}\mathrm{X} \\
\mathrm{X} & \mathrm{x} \\
\mathrm{X} & \\
\mathrm{x} \\
\mathrm{X} \\
\mathrm{X} & \mathrm{x} \\
\mathrm{X} & \\
\mathrm{X} & \mathrm{x}\end{array}$ & $\begin{array}{ll} & \mathrm{x} \\
\mathrm{x} & \mathrm{x} \\
\mathrm{x} \\
\mathrm{X} \\
\mathrm{X} \\
\mathrm{x} \\
\mathrm{x} \\
\mathrm{x} & \mathrm{x}\end{array}$ & & & Zี \\
\hline $17-26.5$ & $\begin{array}{l}3-2,38 \\
3-2,100 \\
3-3,40 \\
3-4,18 \\
3-5,80 \\
3, \text { CC } \\
\end{array}$ & & $\begin{array}{l}\mathrm{X} \\
\mathrm{x}\end{array}$ & 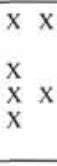 & $\begin{array}{l}x \\
x \\
x \\
x\end{array}$ & & $\begin{array}{rr} & \mathrm{X} \\
\mathrm{x} & \mathrm{X} \\
\mathrm{X} \\
\mathrm{X} & \mathrm{X} \\
\mathrm{X} & \mathrm{X} \\
\mathrm{X} & \mathrm{x} \\
\end{array}$ & $x$ & & $\begin{array}{l}X \\
X \\
X\end{array}$ & & & \\
\hline $26.5-30$ & $\begin{array}{l}4-1,70 \\
4-2,100 \\
4, C C\end{array}$ & & & & $\begin{array}{r}x \\
x \\
\end{array}$ & & $\begin{array}{ll}\mathrm{X} & \mathrm{X} \\
\mathrm{X} & \mathrm{X} \\
\mathrm{X} & \mathrm{X} \\
\end{array}$ & $\begin{array}{ll} & \\
x & x \\
x & x \\
\end{array}$ & & $\begin{array}{l}x \\
x \\
x \\
\end{array}$ & & & \\
\hline \multirow[t]{2}{*}{$36-45.5$} & \multirow{2}{*}{$\begin{array}{l}5-1,130 \\
5-2,60 \\
5-2,115 \\
5-2,128 \\
5-3,115 \\
5-4,50 \\
5-4,54 \\
5-4,64 \\
54,96 \\
5-4,105 \\
5-5,5 \\
5-5,36 \\
5-5,62 \\
5-5,108 \\
5-5,110 \\
5-5,119 \\
5-5,120 \\
5-5,125 \\
5-5,133 \\
5, C C\end{array}$} & \multirow[b]{2}{*}{$\begin{array}{l}x \\
x\end{array}$} & \multirow{2}{*}{\multicolumn{2}{|c|}{$x$}} & $\begin{array}{l}x \\
x\end{array}$ & $\begin{array}{l}\mathrm{x} \\
\mathrm{x} \\
\mathrm{x}\end{array}$ & $\begin{array}{ll}x & \\
x & x \\
x & x \\
& x \\
& x \\
x & x \\
& x \\
x & x\end{array}$ & $\begin{array}{l}\mathrm{x} \\
\mathrm{x} \\
\mathrm{x} \\
\mathrm{x} \\
\mathrm{x} \\
\mathrm{x} \\
\mathrm{x} \\
\mathrm{x}\end{array}$ & & $\begin{array}{r}\mathrm{x} \\
\mathrm{x} \\
\mathrm{x} \\
\mathrm{x} \\
\mathrm{x} \\
\mathrm{x} \\
\mathrm{x}\end{array}$ & & 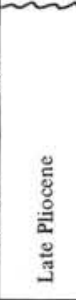 & \begin{tabular}{|l}
$\frac{n}{\frac{n}{2}}$ \\
$\frac{0}{2}$ \\
$\frac{0}{2}$
\end{tabular} \\
\hline & & & & & $\begin{array}{l}x \\
x\end{array}$ & $\mathrm{x}$ & $\begin{array}{ll}\mathrm{X} & \mathrm{X} \\
\mathrm{X} & \mathrm{X} \\
& \mathrm{X} \\
\mathrm{X} & \mathrm{X}\end{array}$ & $\begin{array}{l}\mathrm{X} \\
\mathrm{X} \\
\mathrm{X}\end{array}$ & & $\begin{array}{l}x \\
x \\
x\end{array}$ & & 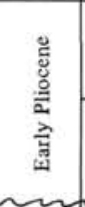 & $\frac{\frac{5}{5}}{\frac{\bar{a}}{4}}$ \\
\hline $45.5-55$ & $\begin{array}{l}6-2,50 \\
6-3,95 \\
6-3,106 \\
6-3,110 \\
6-3,130 \\
6-3,140 \\
6-4,36 \\
6-4,64 \\
6-4,80 \\
6-4,90 \\
6-4,101 \\
6-4,117 \\
6-4,135 \\
6, C C\end{array}$ & $\begin{array}{l}\mathrm{x} \\
\mathrm{x} \\
\mathrm{x} \\
\mathrm{x} \\
\mathrm{x} \\
\\
\mathrm{x} \\
\mathrm{x}\end{array}$ & & mblag & $g e^{x}$ & $\begin{array}{l}x \\
x \\
x\end{array}$ & $\begin{array}{rr}\mathrm{X} & \mathrm{X} \\
\mathrm{X} \\
\mathrm{X} \\
\mathrm{X} \\
\mathrm{X} \\
\mathrm{X} \\
\mathrm{X} \\
\mathrm{X}\end{array}$ & $\begin{array}{l} \\
\mathrm{X} \\
\mathrm{X} \\
\mathrm{X} \\
\mathrm{X} \\
\mathrm{X} \\
\mathrm{X} \\
\mathrm{X} \\
\mathrm{X} \\
\mathrm{X}\end{array}$ & $\mathrm{x}$ & 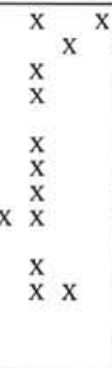 & Mury,rerts & $\underbrace{\text { Pleist. }}_{\text {Messini }}$ & $\frac{1}{2} \frac{a}{a}$ \\
\hline
\end{tabular}

${ }^{\text {a }}$ Probable artificial repetition. 
TABLE 7

Occurrence Ranges of Planktonic Foraminifers at Site 375

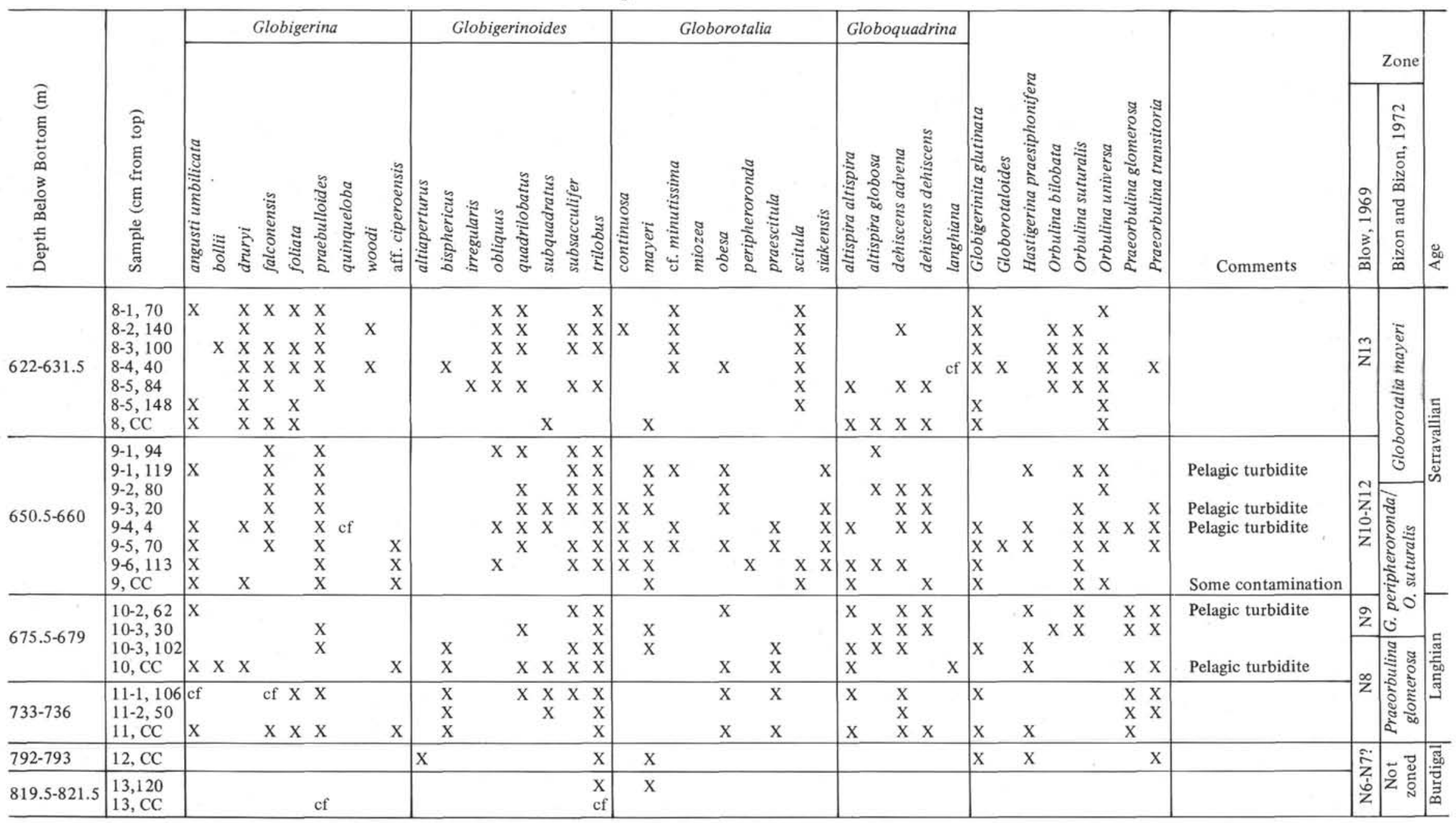


TABLE 8

Occurrence Ranges of Planktonic Foraminifers at Site 377 (cleft in Mediterranean Ridge)

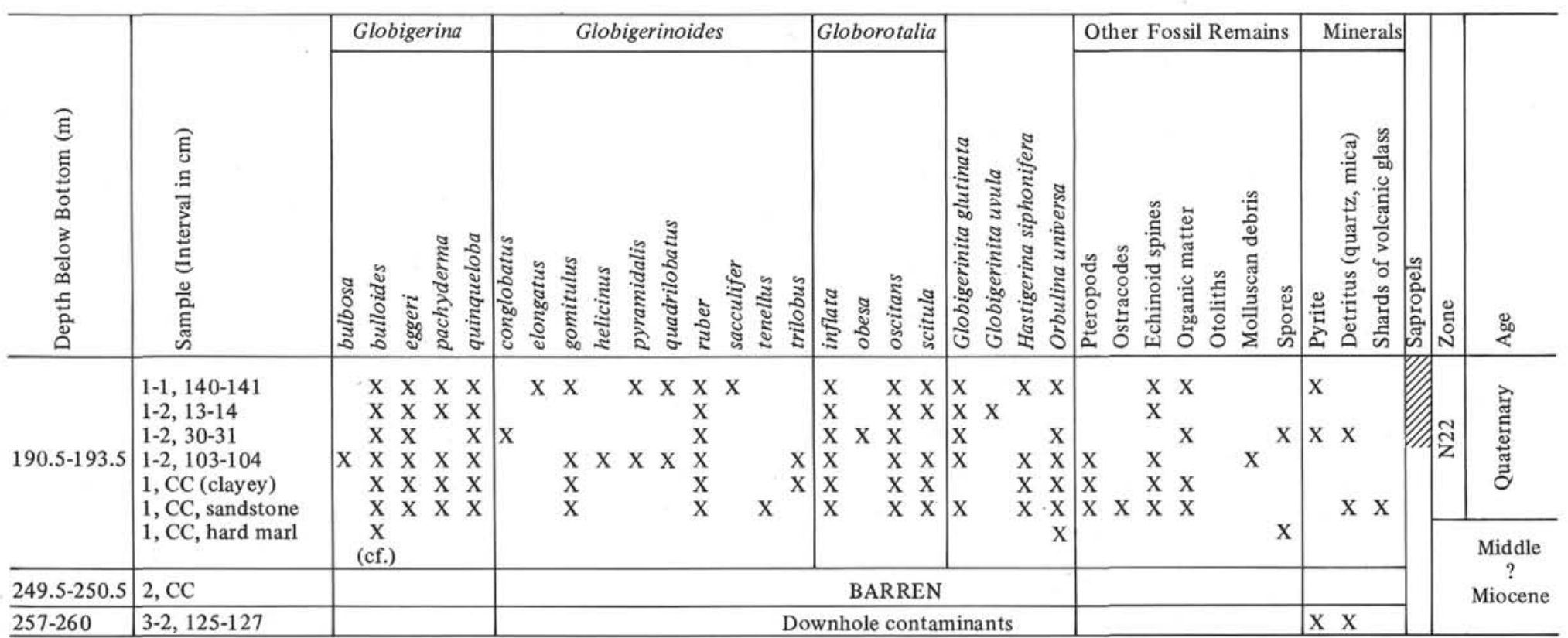


TABLE 9

Occurrence Ranges of Plio-Pleistocene Planktonic Foraminifers and Other Fossils at Hole 378

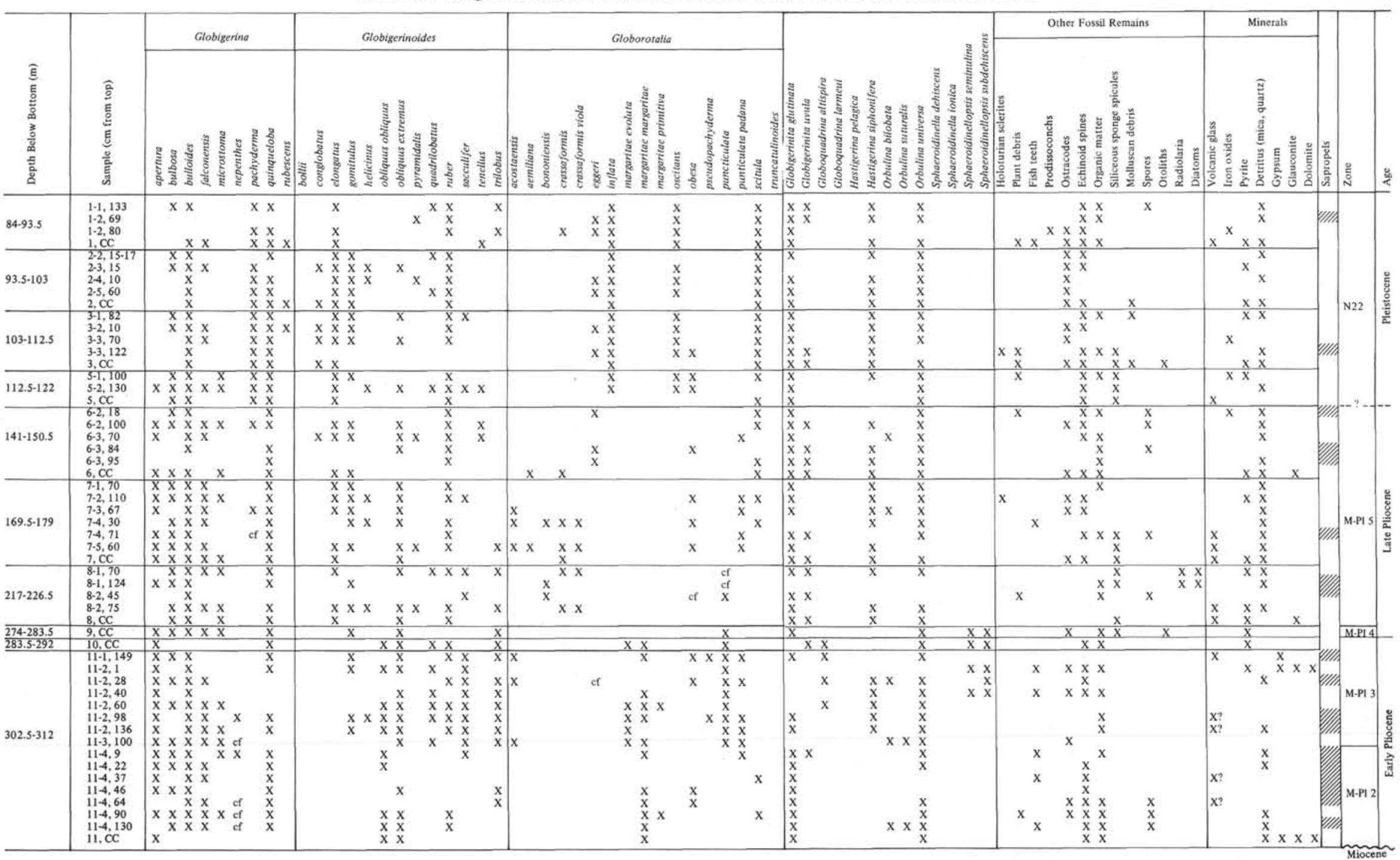


TABLE 10

Occurrence Ranges of Planktonic Foraminifers and Other Fossil Remains at Hole 378A

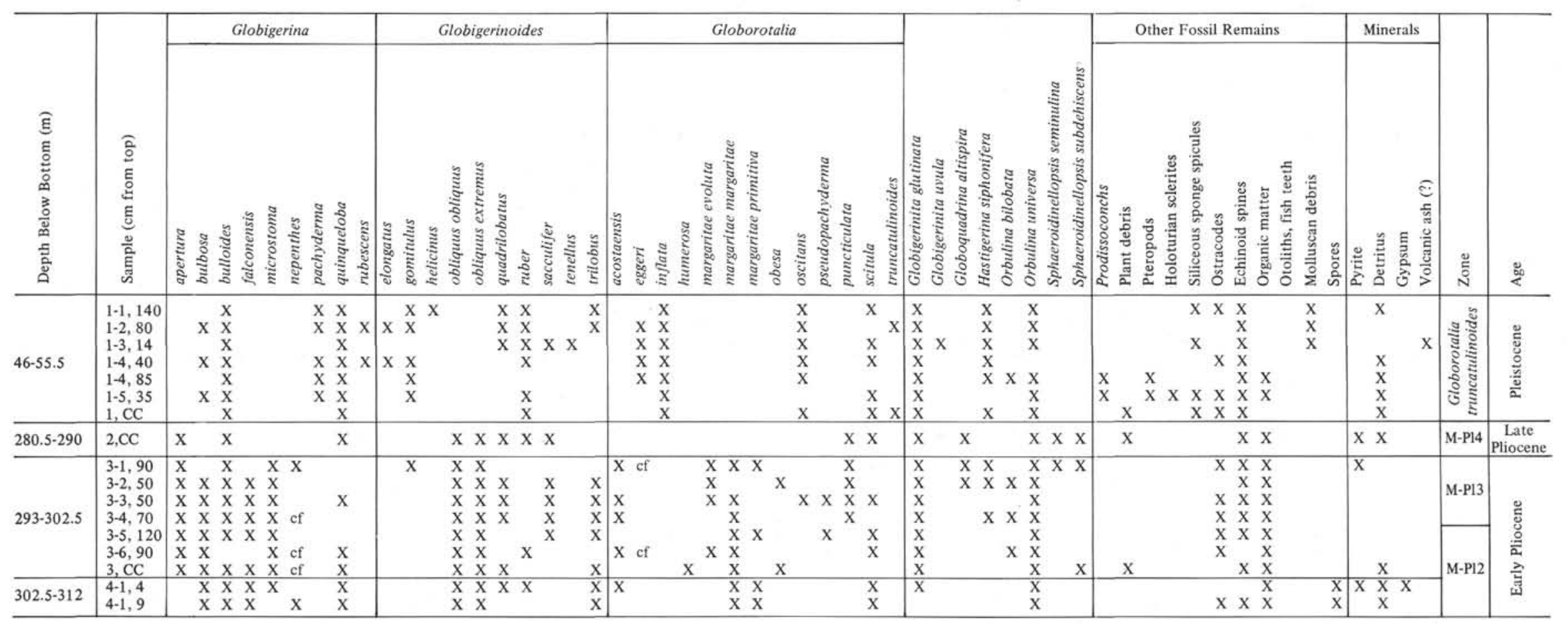


TABLE 11

Planktonic Foraminifers and Other Microfossils at Site 371, Cores 1-8

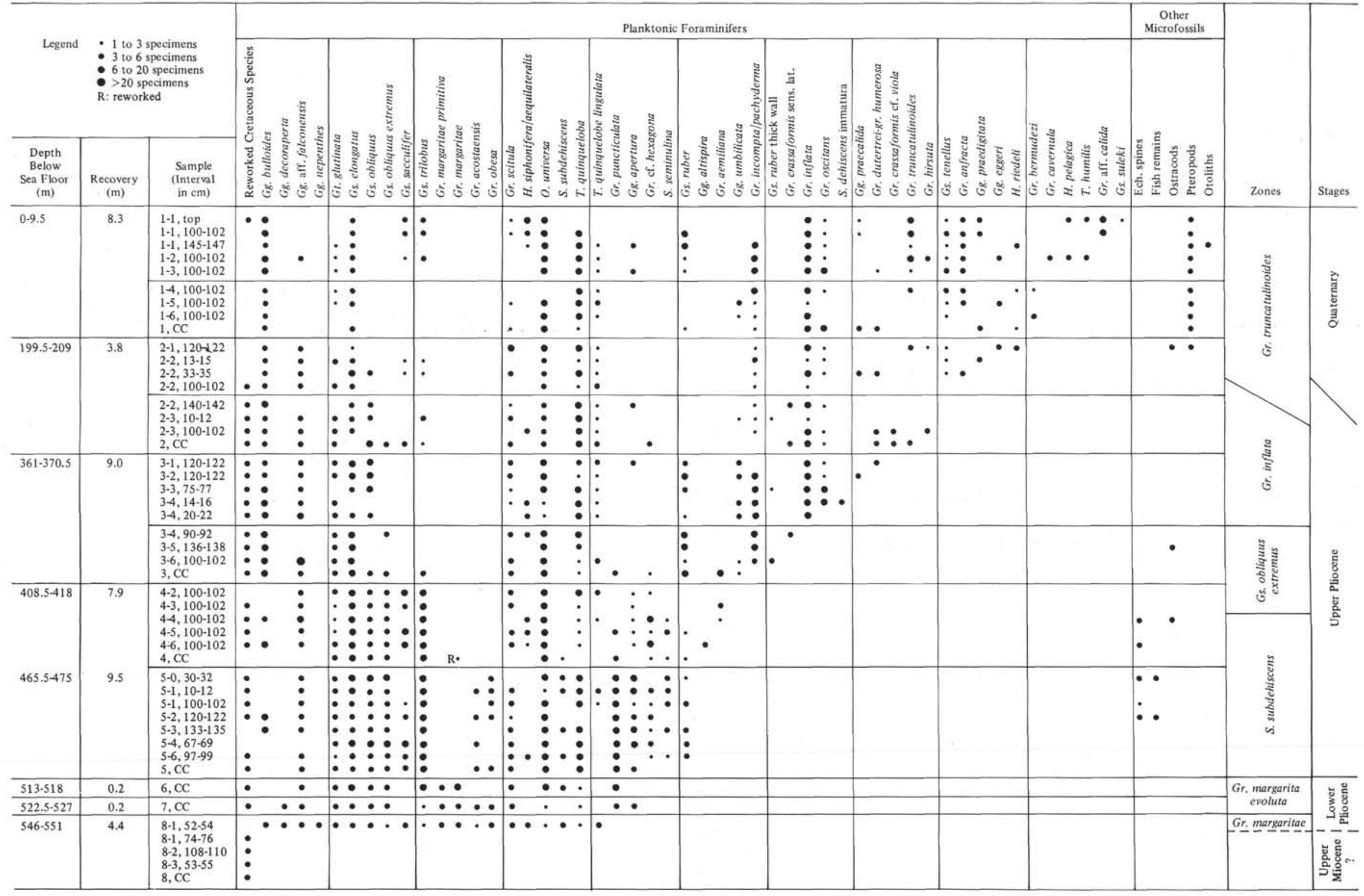


TABLE 12

Planktonic Foraminifers and Other Microfossils at Site 372, Cores $1-6$

\begin{tabular}{|c|c|c|c|c|c|c|c|c|c|c|c|c|c|c|c|}
\hline \multirow{3}{*}{$\begin{array}{c}\text { Legend } \\
\begin{array}{l}\text { Depth } \\
\text { Below } \\
\text { Sea Floor } \\
\text { (m) }\end{array} \\
\end{array}$} & \multirow{2}{*}{\multicolumn{2}{|c|}{$\begin{array}{l}\text { 1 to } 3 \text { specimens } \\
3 \text { to } 6 \text { specimens } \\
: 6 \text { t to } 20 \text { specimens } \\
\bullet>20 \text { specimens } \\
\text { R: reworked }\end{array}$}} & \multicolumn{10}{|c|}{ Planktonic Foraminifers } & \multirow{3}{*}{ 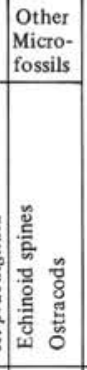 } & \multirow[b]{3}{*}{ Zones } & \multirow[b]{3}{*}{ Stages } \\
\hline & & & \multirow{2}{*}{ 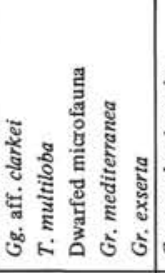 } & \multirow{2}{*}{ 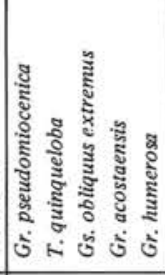 } & \multirow{2}{*}{ 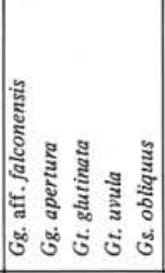 } & \multirow{2}{*}{ 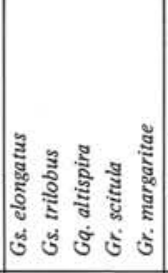 } & \multirow{2}{*}{ 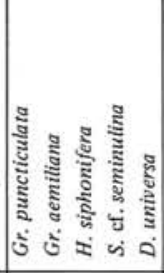 } & \multirow{2}{*}{ 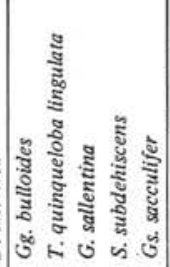 } & \multirow{2}{*}{ 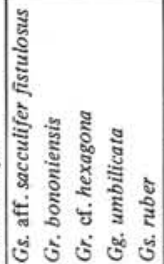 } & \multirow{2}{*}{ 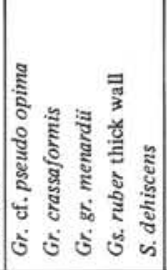 } & \multirow{2}{*}{ 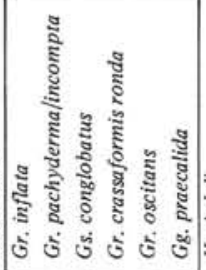 } & \multirow{2}{*}{ 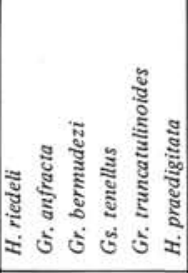 } & & & \\
\hline & $\begin{array}{c}\text { Recovery } \\
(\mathrm{m})\end{array}$ & $\begin{array}{c}\text { Sample } \\
\text { (Interval } \\
\text { in } \mathrm{cm})\end{array}$ & & & & & & & & & & & & & \\
\hline $112-121.5$ & 4.2 & $\begin{array}{l}1-1,140-142 \\
1-2,90-92 \\
1-3,100-102 \\
1-4,30-32 \\
1, \mathrm{CC}\end{array}$ & & $:$ & $\begin{array}{l}: \because \\
\mathrm{R} \cdot\end{array}$ & 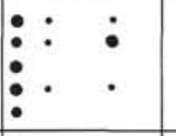 & $\begin{array}{l}: \\
: \\
\bullet \\
\end{array}$ & $::$ & $\begin{array}{r}0: \\
: \\
0\end{array}$ & : & $:: \vdots::$ & $\begin{array}{l}\bullet: \vdots \\
\vdots \\
0\end{array}$ & & 1 & 졀 \\
\hline $131-140.5$ & 5.2 & $\begin{array}{l}2-1,117-119 \\
2-2,27-29 \\
2-2,100-102 \\
2-2,135-137 \\
2-3,41-43 \\
2-3,120-122 \\
\end{array}$ & & $\because:$ & 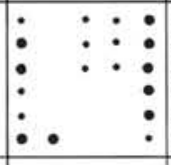 & $::$ & \begin{tabular}{rl|}
$\mathrm{R}$ & $:$ \\
$\mathrm{R} \cdot$ & $\vdots$ \\
$\cdot$ & $\vdots$ \\
\end{tabular} & $: \because: \quad:$ & $\begin{array}{r}\bullet: \\
\vdots \\
\bullet\end{array}$ & $\begin{array}{r}\cdot: \\
: \\
:\end{array}:$ & 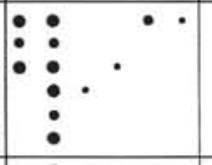 & & - & ปั้ & \multirow{3}{*}{ 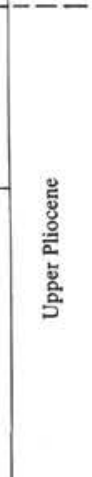 } \\
\hline & & $\begin{array}{l}2-3,126-128 \\
2-4,76-78 \\
2-4,106-108 \\
2-4,110-112 \\
2, C C \\
\end{array}$ & & $\dot{:}$ & \begin{tabular}{ll|}
$:$ & $\vdots$ \\
$:$ & $:$ \\
\end{tabular} & 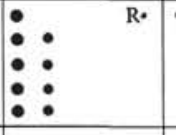 & $\begin{array}{ll}: & : \\
\vdots & :\end{array}$ & : & \begin{tabular}{rr|}
$\cdot \quad \therefore$ \\
& $\vdots$ \\
\end{tabular} & 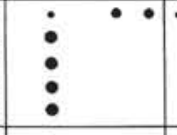 & $\bullet$ & & - & 气ूँ & \\
\hline \multirow[t]{2}{*}{$149.5-150$} & 4.3 & $\begin{array}{l}3-1,48-50 \\
3-1,65-67 \\
3-1,139-141 \\
3-2,24-26 \\
3-2,75-77 \\
3-2,100-102 \\
3-2,115-117 \\
\end{array}$ & & $: \vdots$ & : : : : : & : : & 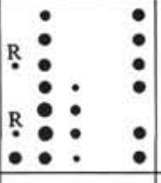 & $:$ & 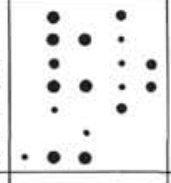 & $\because$ & & & . & 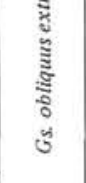 & \\
\hline & & $\begin{array}{l}3-2,142-144 \\
3-3,6-8 \\
3-3,20-22 \\
3-3,84-86 \\
3-3,100-102 \\
3-3,116-118 \\
3, \text { CC } \\
\end{array}$ & & 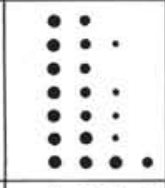 & 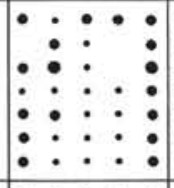 & 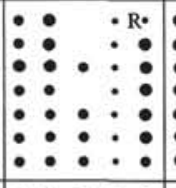 & 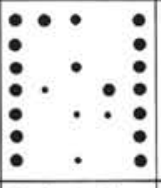 & $\begin{array}{l}: \\
: \\
\vdots \\
\vdots\end{array}$ & $\cdots$ & & & & $\cdot$ & 2 & 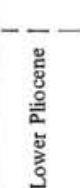 \\
\hline $150-159.5$ & 2.5 & $\begin{array}{l}4-1,59-61 \\
4-1,64-65 \\
4-1,90-92 \\
4-1,140-142 \\
4-2,1-3 \\
4-2,100-102 \\
4, C C \\
\end{array}$ & $:$ & $\begin{array}{l}\vdots \cdots \\
\bullet \cdot\end{array}$ & $\bullet \bullet \bullet$ & $\bullet \bullet \bullet \bullet$ & $\bullet \cdot \bullet$ & & & & & & & \multirow{3}{*}{ 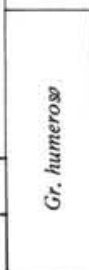 } & \multirow{3}{*}{ 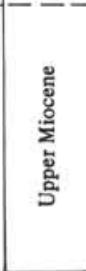 } \\
\hline 159.5-169 & 0.4 & $\begin{array}{l}5-1,132-133 \\
5, \mathrm{CC}\end{array}$ & $\bullet$ & & & & & & & & & & & & \\
\hline $169-178.5$ & 0.4 & $\begin{array}{l}6-1,142-144 \\
6, \mathrm{CC}\end{array}$ & $\cdot \cdot$ & & & & & & & & & & & & \\
\hline
\end{tabular}


TABLE 13

Planktonic Foraminifers and Other Microfossils at Site 372, Cores 9-15

\begin{tabular}{|c|c|c|c|c|c|c|c|c|c|c|c|c|}
\hline \multirow[b]{2}{*}{ Legend } & \multirow{2}{*}{\multicolumn{2}{|c|}{$\begin{array}{l}: 1 \text { to } 3 \text { specimens } \\
3 \text { to } 6 \text { specimens } \\
6 \text { to } 20 \text { specimens } \\
\text { R: reworked }\end{array}$}} & \multicolumn{7}{|c|}{ Planktonic Foraminifers } & \multirow{3}{*}{ Other Microfossils } & \multirow[b]{3}{*}{ Zones } & \multirow[b]{3}{*}{ Stages } \\
\hline & & & \multirow{2}{*}{ 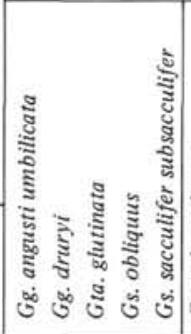 } & \multirow{2}{*}{ 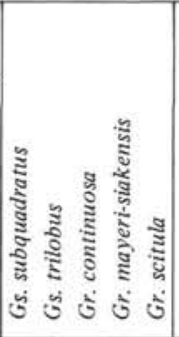 } & \multirow{2}{*}{ 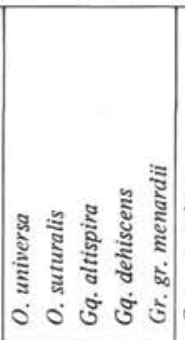 } & \multirow{2}{*}{ 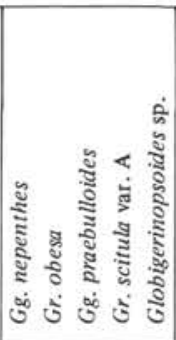 } & \multirow{2}{*}{ 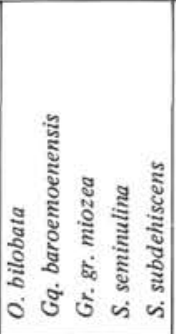 } & \multirow{2}{*}{ 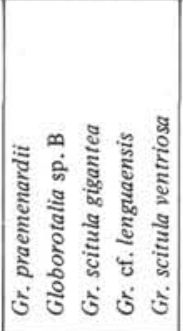 } & \multirow{2}{*}{ 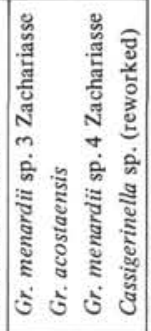 } & & & \\
\hline $\begin{array}{l}\text { Depth } \\
\text { Below } \\
\text { Sea Floor } \\
\text { (m) } \\
\end{array}$ & $\begin{array}{c}\text { Recovery } \\
(\mathrm{m})\end{array}$ & $\begin{array}{c}\text { Sample } \\
\text { (Interval } \\
\text { in } \mathrm{cm})\end{array}$ & & & & & & & & & & \\
\hline \multirow[t]{2}{*}{$197.5-207$} & \multirow[t]{2}{*}{5.4} & $\begin{array}{l}9-1,96-98 \\
9-1,130-132 \\
9-1,140-142 \\
9-1,148-149 \\
9-2,28-30\end{array}$ & $\therefore$ & 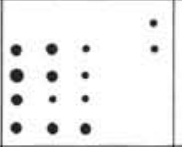 & : & : & - & $:$ & : $\bullet:$ & $:$ & \multirow{3}{*}{ 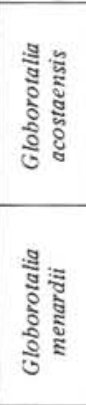 } & \multirow{3}{*}{ 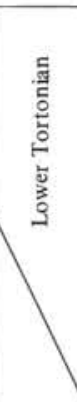 } \\
\hline & & $\begin{array}{l}9-2,75-77 \\
9-2,110-112 \\
9-3,20-22 \\
9-4,100-102 \\
9, \text { CC }\end{array}$ & 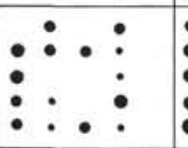 & $\because::^{\mathrm{R}}{ }^{\prime}$ & $:: \bullet$ & $: \because$ & •・ & & $: \bullet$ & : & & \\
\hline $207-216.5$ & 2.5 & $\begin{array}{l}10-1,120-122 \\
10-2,5-7 \\
10-2,100-102 \\
10, \mathrm{CC}\end{array}$ & $: \bullet: \vdots$ & 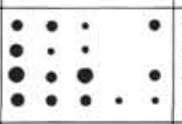 & $\begin{array}{lllll}\bullet & \bullet & \bullet & \bullet & \bullet \\
\bullet & \bullet & \bullet & \bullet & \\
\bullet & \bullet & \bullet & & \bullet\end{array}$ & $\begin{array}{r}\bullet: \bullet \\
\bullet: \\
\bullet:\end{array}$ & $\begin{array}{l}\bullet \bullet \\
\bullet \cdot\end{array}$ & $\begin{array}{lll}\cdot . & & \\
& & \\
& \cdot\end{array}$ & : & : & & \\
\hline $216.5-226$ & 3.9 & $\begin{array}{l}11-1,120-122 \\
11-2,100-102 \\
11-3,100-102 \\
11-3,140-142 \\
11, \propto C\end{array}$ & $\begin{array}{llll}\bullet & \vdots & \bullet & \bullet \\
\vdots & \bullet & & \bullet \\
& \bullet & \bullet & \end{array}$ & 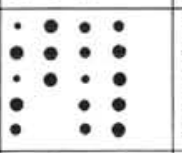 & 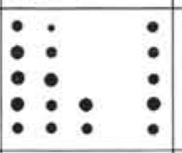 & $\begin{array}{lll}\bullet: & \bullet \\
\bullet & \bullet\end{array}$ & $: \vdots$ & $\bullet$ & & $: \quad:$ & & \\
\hline $226-235.5$ & 6.5 & $\begin{array}{l}12-1,13-15 \\
12-3,110-120 \\
12-3,128-130 \\
12-4,100-102 \\
12-5,100-102 \\
12-6,100-102 \\
12, \text { CC }\end{array}$ & 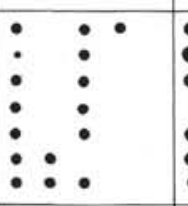 & 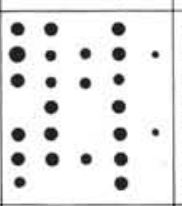 & 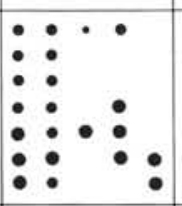 & $\begin{array}{lll}\bullet & \bullet \\
0 & \bullet \\
\bullet & & \end{array}$ & $\begin{array}{r}\bullet \\
\bullet \quad \bullet \\
\bullet \\
\bullet\end{array}$ & 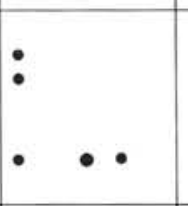 & & 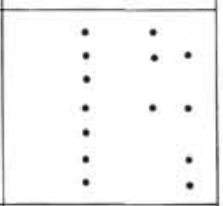 & & \\
\hline $235.5-245$ & 9.2 & $\begin{array}{l}13-1,100-102 \\
13-2,100-102 \\
13-3,100-102 \\
13-4,100-102 \\
13-5,100-102 \\
13-6,100-102 \\
13, \text { CC }\end{array}$ & $\begin{array}{lll}\bullet & \bullet & \\
\vdots & \vdots & \bullet \\
\vdots & \bullet & \\
& \bullet & \end{array}$ & $\begin{array}{lllll}\bullet & \bullet & \bullet & \bullet & \bullet \\
\bullet & \bullet & & \bullet & \bullet \\
\bullet & \bullet & \bullet & \bullet & \cdot \\
\bullet & \bullet & \bullet & \bullet \\
\bullet & \bullet & & & \end{array}$ & 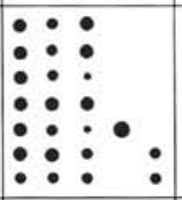 & 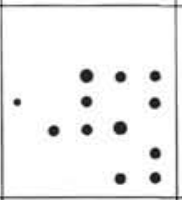 & $\begin{array}{l}\bullet \\
\bullet \\
\bullet\end{array}$ & • & & 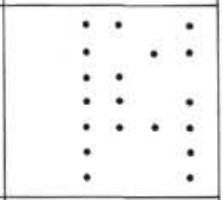 & 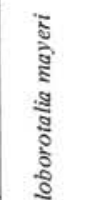 & 㱖 \\
\hline $245-254.5$ & 8.6 & $\begin{array}{l}14-1,100-102 \\
14-2,100-102 \\
14-3,100-102 \\
14-4,90-92 \\
14-5,100-102 \\
14-6,100-102 \\
14, \mathrm{CC}\end{array}$ & 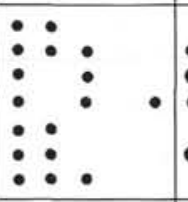 & 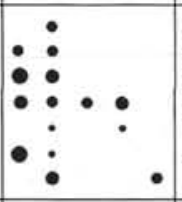 & 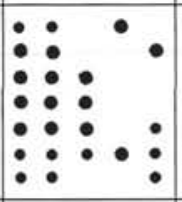 & 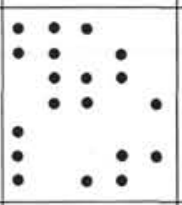 & $\bullet \bullet \cdot \bullet$ & : & & 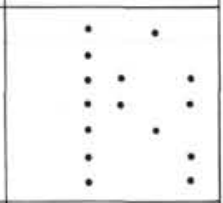 & & \\
\hline $254.5-264$ & 7.7 & $\begin{array}{l}15-2,100-102 \\
15-3,100-102 \\
15-4,100-102 \\
15-5,100-102 \\
15-6,100-102 \\
15, \text { CC }\end{array}$ & 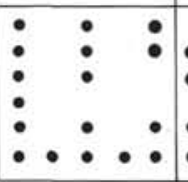 & $\begin{array}{lllll}\bullet & \bullet & \bullet & \bullet & \bullet \\
\bullet & \bullet & \bullet & \bullet & \bullet \\
\bullet & \bullet & \bullet & \bullet & \bullet \\
\bullet & \bullet & \bullet & \bullet\end{array}$ & 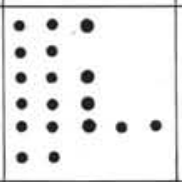 & $: \vdots: \vdots: \bullet$ & $\bullet$ & & & $\begin{array}{ll}\vdots \\
\vdots & \vdots \\
\vdots & \\
\end{array}$ & & \\
\hline
\end{tabular}


TABLE 15

Planktonic Foraminifers and Other Microfossils at Site 372, Cores 24-32

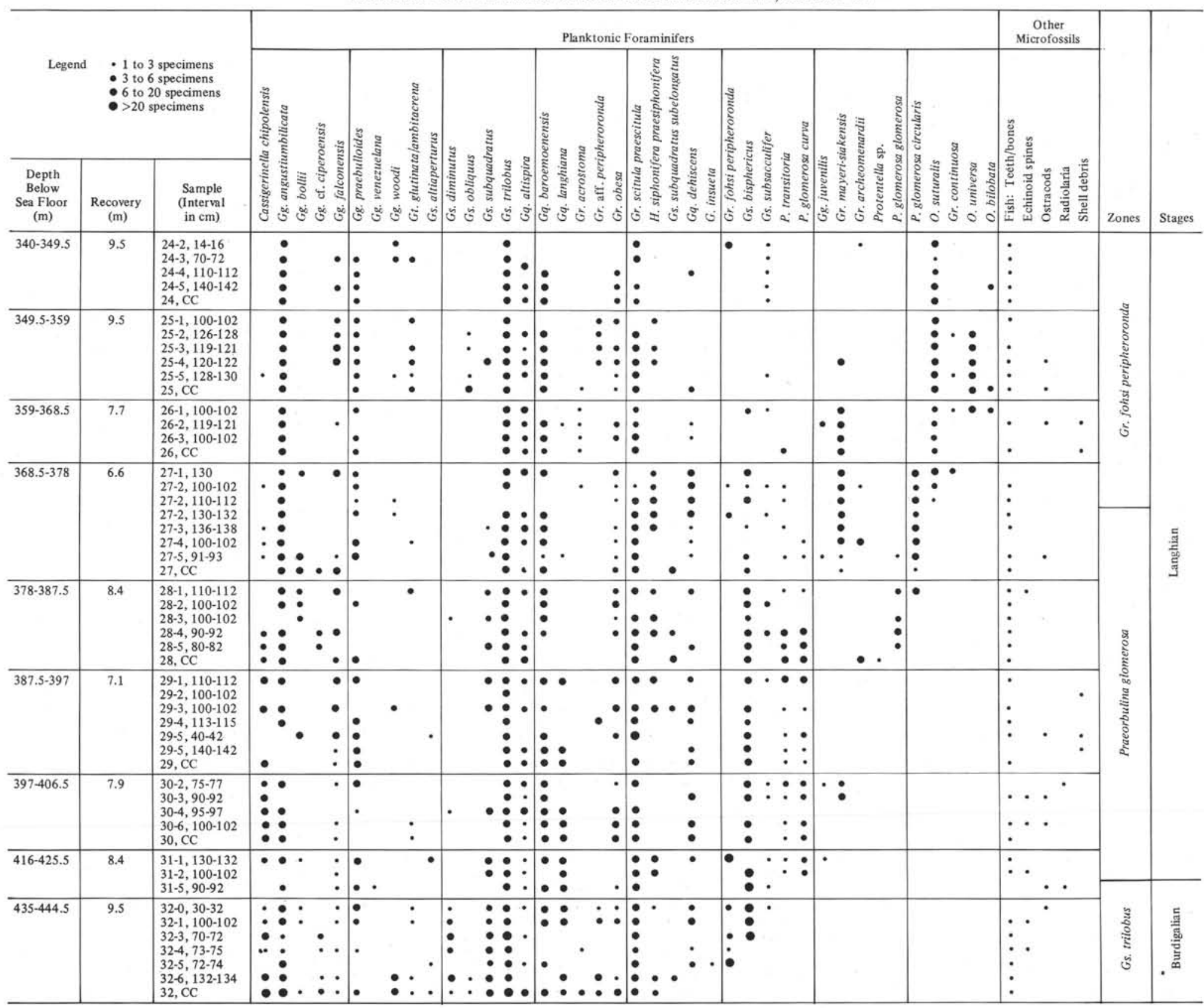


G. BIZON, M. B. CITA, R. WRIGHT, C. MUULLER

TABLE 16

Planktonic Foraminifers and Other Microfossils at Site 372, Cores 33-46

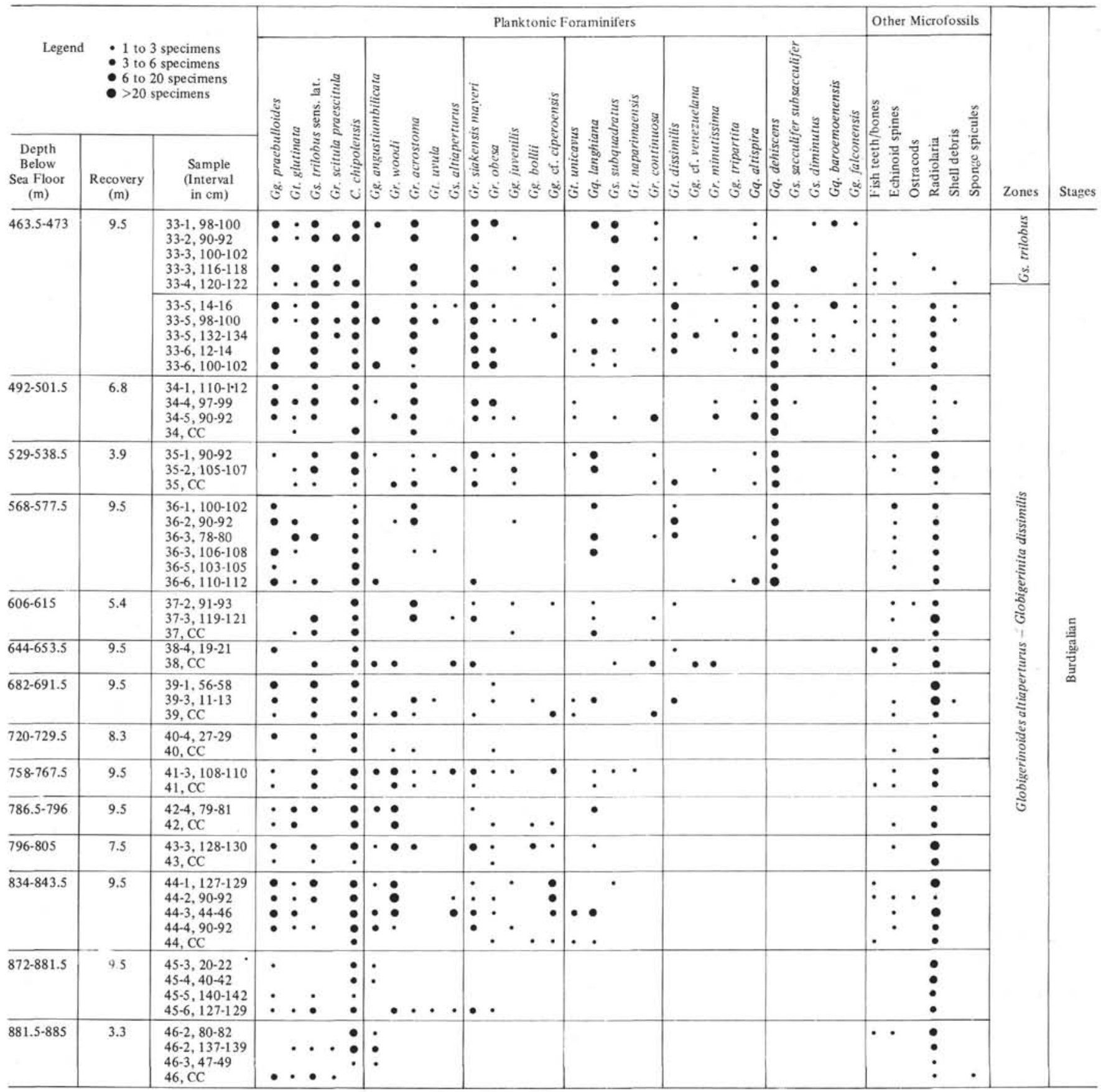


TABLE 17

Planktonic Foraminifers and Other Microfossils at Site 374, Cores 1-7

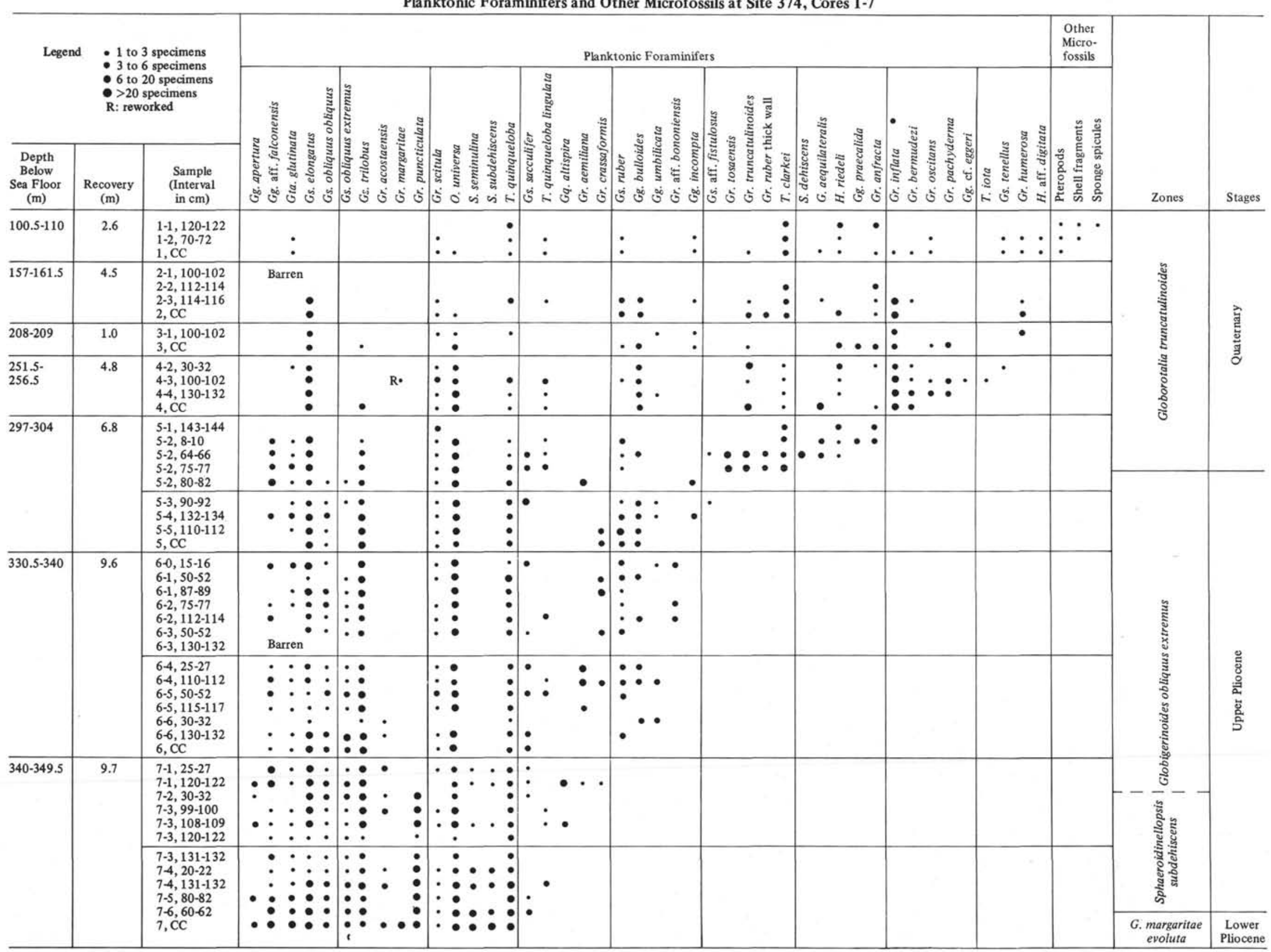


G. BIZON, M. B. CITA, R. WRIGHT, C. MƯLLER

TABLE 18

Planktonic Foraminifers and Other Microfossils at Site 374, Cores 8-15

\begin{tabular}{|c|c|c|c|c|c|c|c|c|c|}
\hline \multirow{2}{*}{\multicolumn{3}{|c|}{ Legend }} & \multicolumn{4}{|c|}{ Planktonic Foraminifers } & $\begin{array}{c}\text { Other } \\
\text { Microfossils }\end{array}$ & \multirow{3}{*}{ Zones } & \multirow[b]{3}{*}{ Stages } \\
\hline & & & \multirow{2}{*}{ 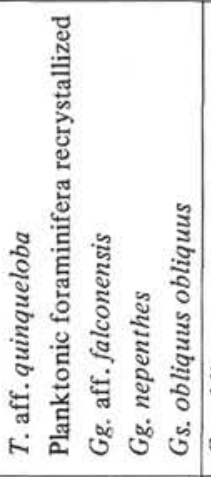 } & \multirow{2}{*}{ 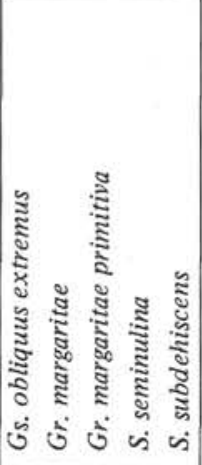 } & \multirow{2}{*}{ 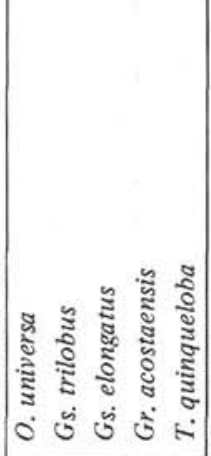 } & \multirow[b]{2}{*}{ 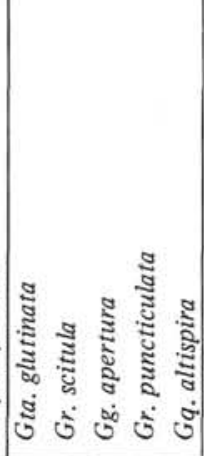 } & \multirow{2}{*}{ 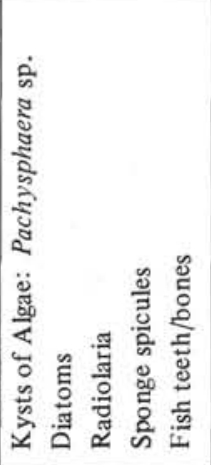 } & & \\
\hline $\begin{array}{l}\text { Depth } \\
\text { Below } \\
\text { Sea Floor } \\
(\mathrm{m})\end{array}$ & $\begin{array}{l}\text { Recovery } \\
\text { (m) }\end{array}$ & $\begin{array}{c}\text { Sample } \\
\text { (Interval } \\
\text { in } \mathrm{cm} \text { ) }\end{array}$ & & & & & & & \\
\hline $349.5-359$ & 5.5 & $\begin{array}{l}8-1,120-122 \\
8-2,122-124 \\
8-3,20-21 \\
8-3,26-27 \\
8-3,80-81 \\
8-4,20-21 \\
8-4,110-111 \\
8, \mathrm{CC} \\
\end{array}$ & $\begin{array}{ll}: & : \\
: & : \\
: & : \\
: & \vdots \\
& :\end{array}$ & 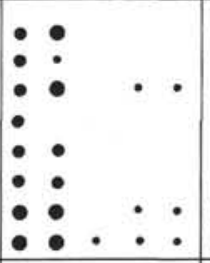 & 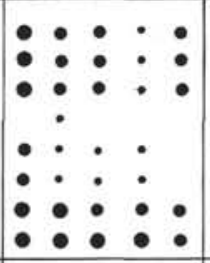 & 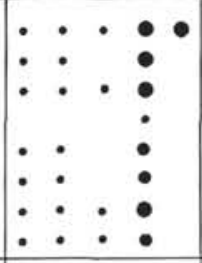 & & 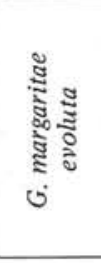 & \multirow{3}{*}{ 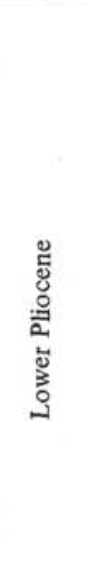 } \\
\hline $359-368.5$ & 6.1 & $\begin{array}{l}9-1,51-52 \\
9-1,148-149 \\
9-2,33-34 \\
9-2,131-132 \\
9-3,7-8 \\
9-3,108-109 \\
9-4,125-127 \\
9, \mathrm{CC} \\
\end{array}$ & 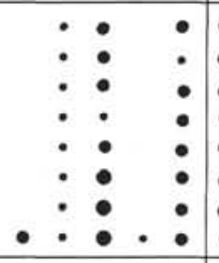 & 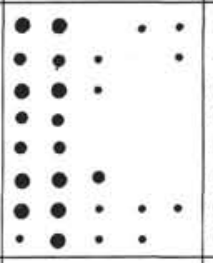 & $\begin{array}{lllll}\bullet & \bullet & \bullet & \bullet & \bullet \\
\bullet & \bullet & \bullet & \bullet & \bullet \\
\bullet & \bullet & \bullet & \bullet & \bullet \\
\bullet & \bullet & \bullet & \bullet & \bullet \\
\bullet & \bullet & \bullet & \bullet & \bullet \\
\bullet & \bullet & \bullet & \bullet & \bullet\end{array}$ & 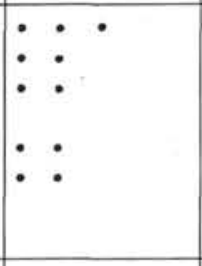 & . & \multirow[t]{2}{*}{$\begin{array}{l}\text { ปัँ } \\
\text { :ิ } \\
\text { ปี } \\
\text { ปे }\end{array}$} & \\
\hline $368.5-378$ & 0.7 & $\begin{array}{l}10-1,110-112 \\
10, C C\end{array}$ & $\bullet: \bullet \bullet: \bullet$ & $\begin{array}{lllll}\bullet & \bullet & \bullet & \bullet & \\
\bullet & \bullet & \bullet & \bullet\end{array}$ & $\bullet \bullet \bullet$ & & & & \\
\hline $378-381.5$ & 2.5 & $\begin{array}{l}11-1,90-91 \\
11-1,138-139 \\
11-2,20-22 \\
11-2,80-82 \\
11-2,114-116 \\
11-2,130-132 \\
11, \text { CC } \\
\end{array}$ & $\bullet$ & & & & & \multirow[t]{5}{*}{ a. } & \multirow{5}{*}{ 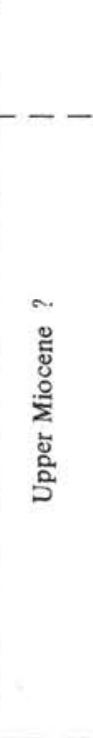 } \\
\hline $\begin{array}{l}381.5- \\
387.5\end{array}$ & 2.5 & $\begin{array}{l}12-1,51-52 \\
12-1,97-98 \\
12-2,120-122 \\
12, \mathrm{CC}\end{array}$ & $\dot{.}$ & & & & & & \\
\hline $\begin{array}{l}387.5- \\
392.5\end{array}$ & 4.1 & $\begin{array}{l}13-1,123-124 \\
13-2,20-22 \\
13-3,103-105 \\
13, \text { CC }\end{array}$ & • & & & & $\cdot$ & & \\
\hline $392.5-397$ & 3.3 & $\begin{array}{l}14-1,110-112 \\
14-2,132-134 \\
14, \mathrm{CC}\end{array}$ & & & & & - & & \\
\hline $397-406.5$ & 3.1 & $\begin{array}{l}15-1,30-32 \\
15-2,100-102 \\
15-2,147-148 \\
15, \text { CC }\end{array}$ & $\cdot$ & & & & - $\bullet$ & & \\
\hline
\end{tabular}


TABLE 19

Planktonic Foraminifers and Other Microfossils at Site 375, Cores 1-7

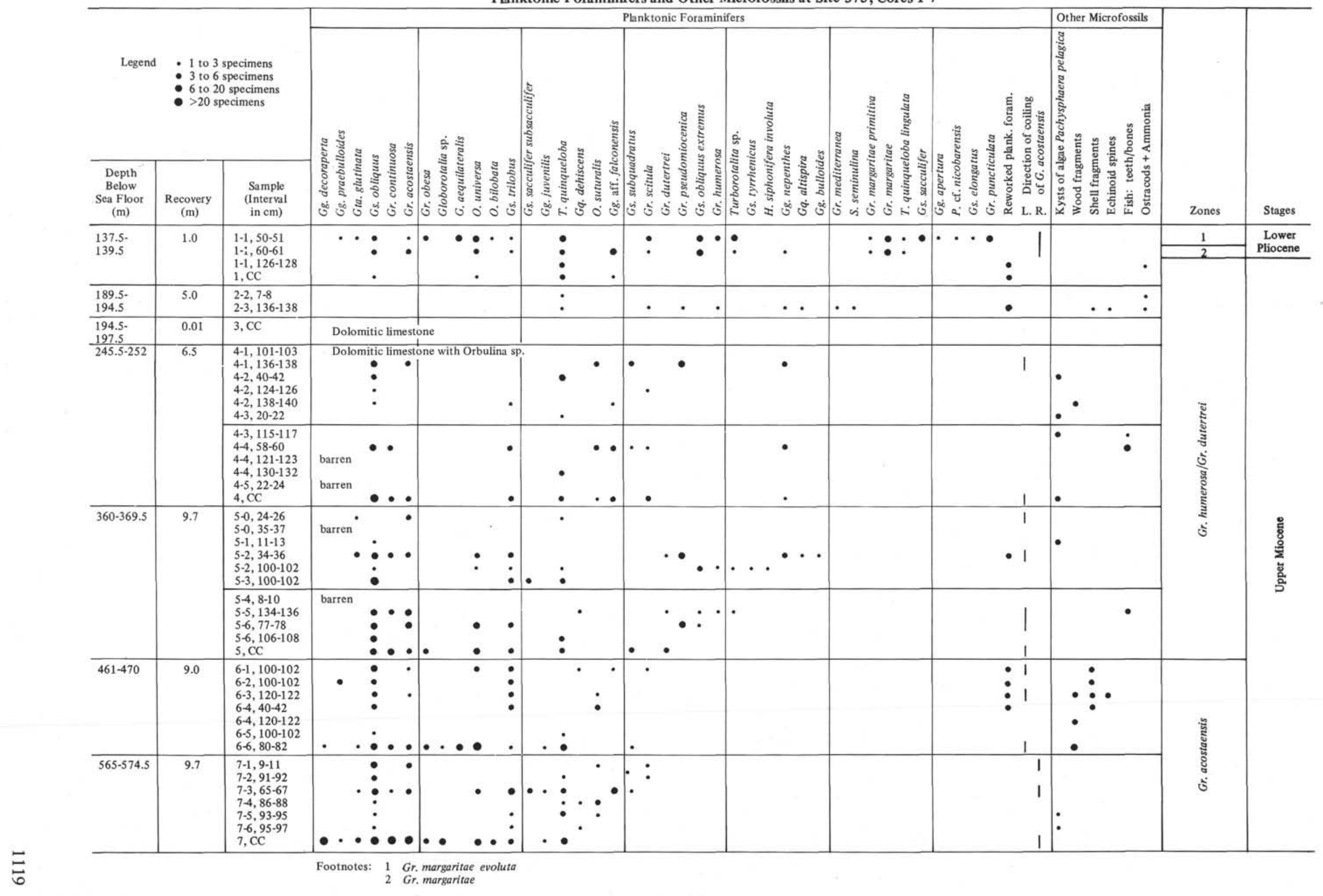


TABLE 20

Planktonic Foraminifers and Other Microfossils at Site 375, Cores 8-13

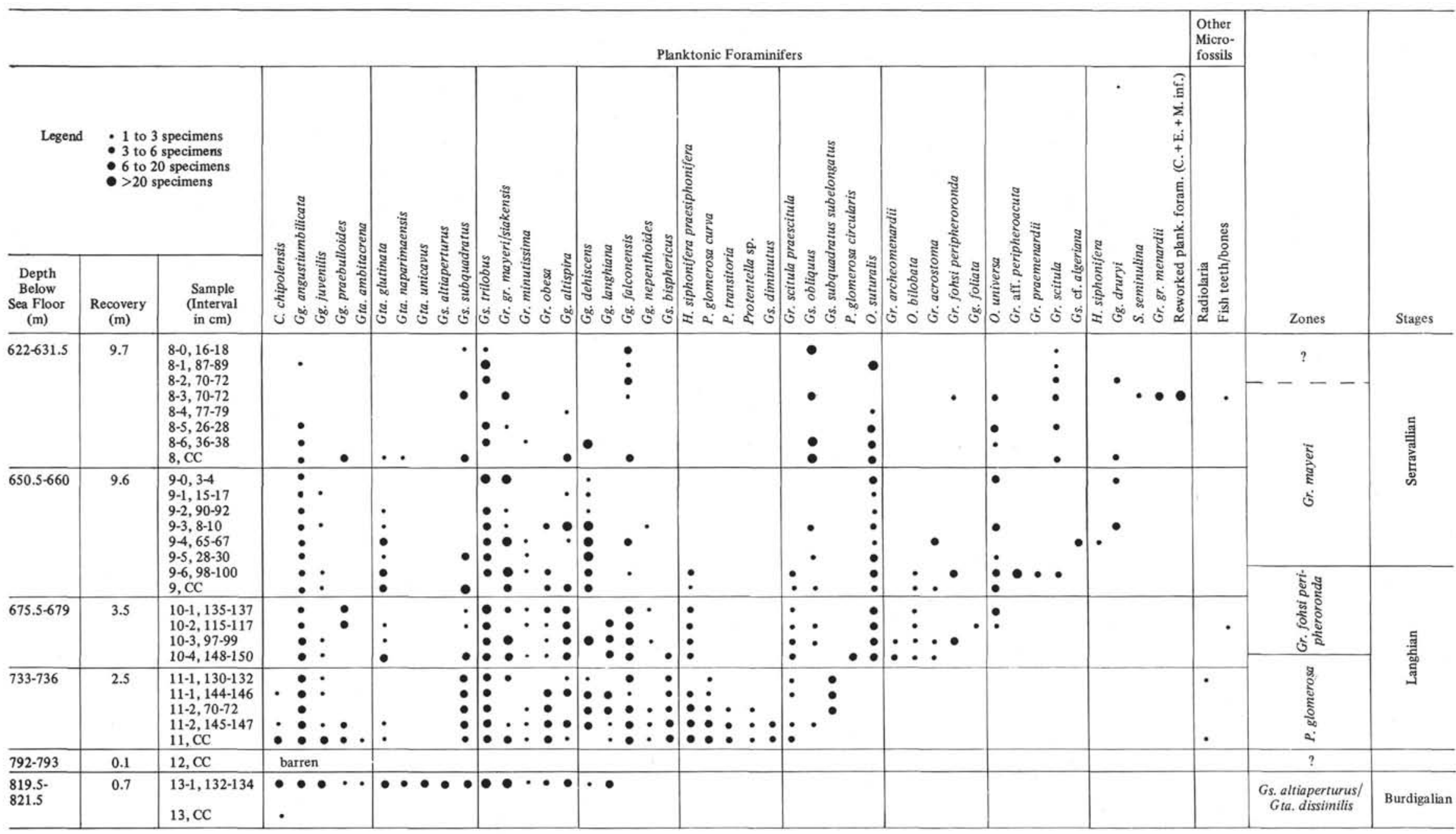


TABLE 21

Planktonic Foraminifers and Other Microfossils at Site 376, Cores 1-5

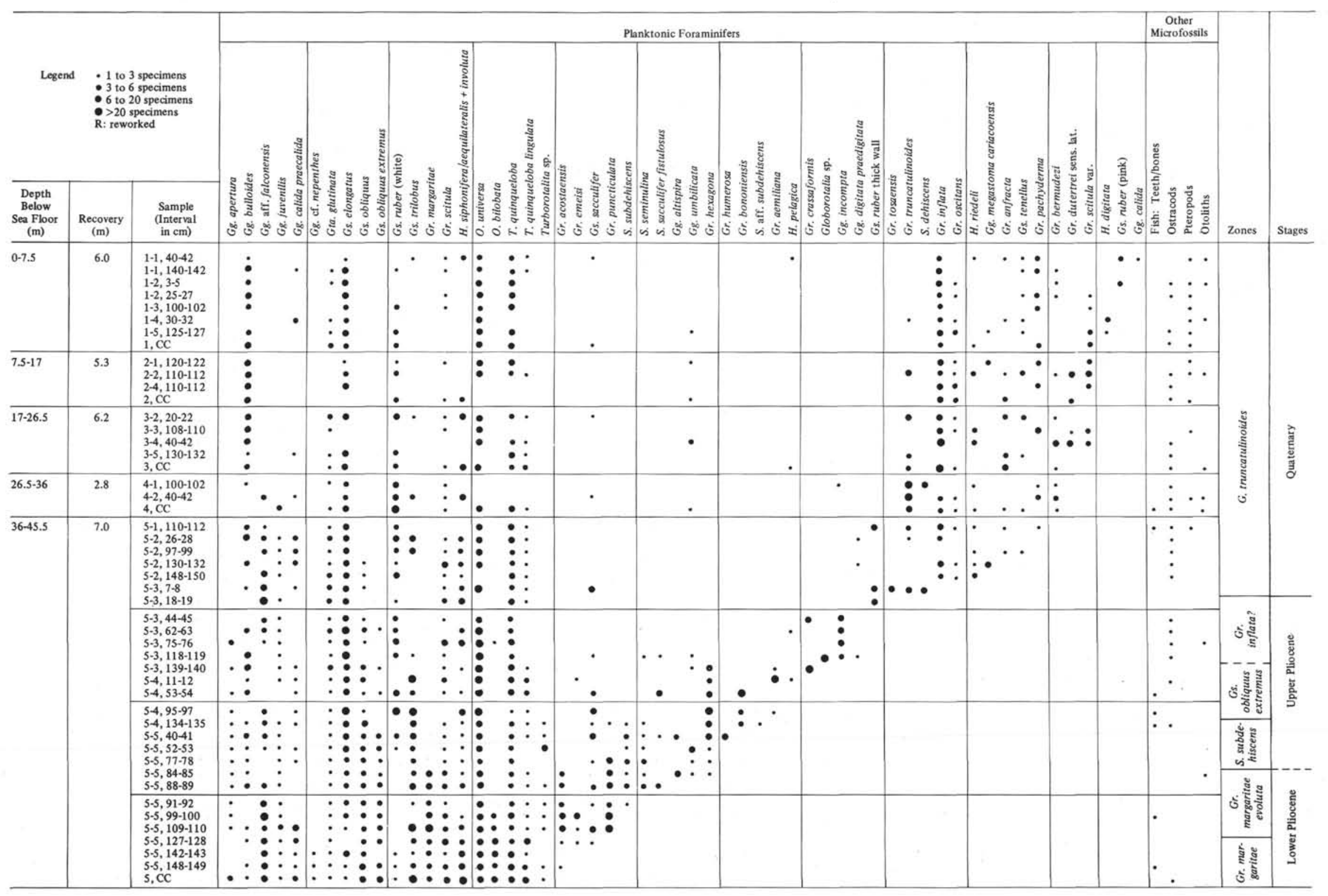


G. BIZON, M. B. CITA, R. WRIGHT, C. MÜLLER

TABLE 22

Planktonic Foraminifers and Other Microfossils at Site 376, Cores 6-15

\begin{tabular}{|c|c|c|c|c|c|c|c|c|c|c|c|c|}
\hline \multirow[b]{2}{*}{ Legend } & \multirow{2}{*}{\multicolumn{2}{|c|}{$\begin{array}{l}: 1 \text { to } 3 \text { specimens } \\
: 3 \text { to } 6 \text { specimens } \\
: 6 \text { to } 20 \text { specimens } \\
->20 \text { specimens }\end{array}$}} & \multicolumn{6}{|c|}{ Planktonic Foraminifers } & \multicolumn{2}{|c|}{ Other Microfossils } & \multirow[b]{2}{*}{ Zones } & \multirow[b]{2}{*}{ Stages } \\
\hline & & & 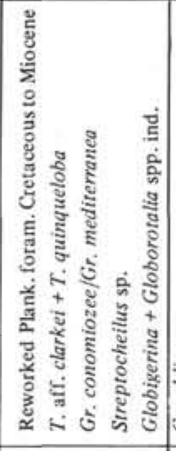 & 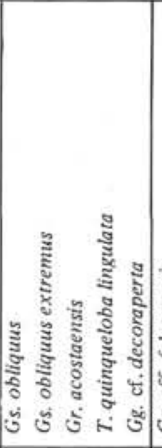 & 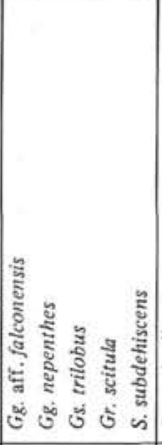 & 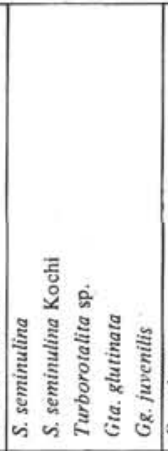 & 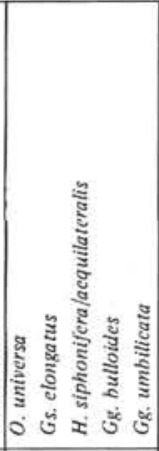 & 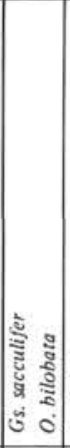 & 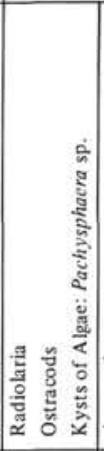 & 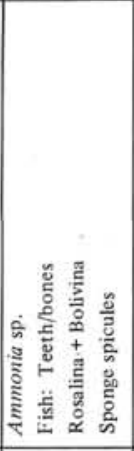 & & \\
\hline \multirow[t]{4}{*}{$45.5-55$} & \multirow[t]{4}{*}{5.1} & $\begin{array}{l}6-1 \text { to } 6-3,90 \\
6-3,104-105 \\
6-3,133-134 \\
6-3,149-150 \\
6-4,12-13 \\
6-4,33-34\end{array}$ & & $\because: \vdots:$ & 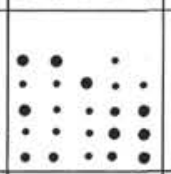 & : $: \vdots: \vdots:$ & $: \vdots:$ & $\bullet$ & & \begin{tabular}{|r|} 
\\
\end{tabular} & 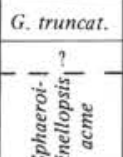 & \multirow{2}{*}{ 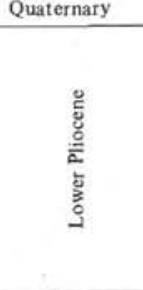 } \\
\hline & & $\begin{array}{l}6-4,56-57 \\
6-4,66-67 \\
6-4,80-81 \\
6-4,83-84 \\
6-4,85-86 \\
\end{array}$ & : & $\begin{array}{l}\bullet: \\
\vdots \\
\vdots\end{array}$ & $\because \vdots:$ & $\mid \begin{array}{ccc}\bullet & & \bullet \\
& \vdots & \vdots \\
\bullet & \vdots & \bullet\end{array}$ & $\begin{array}{l}: \bullet \cdot \\
:\end{array}$ & & & 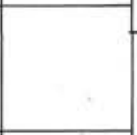 & के ฐิ & \\
\hline & & \multirow{2}{*}{$\begin{array}{l}6-4,89-90 \\
6-4,108-109 \\
6-4,125-126 \\
6-4,137-138 \\
6-4,140-141 \\
6, \mathrm{CC} \\
\end{array}$} & \multirow{2}{*}{$:$} & \multirow{2}{*}{ 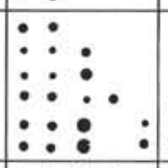 } & \multirow{2}{*}{ 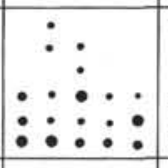 } & \multirow{2}{*}{$\begin{array}{c}\vdots \\
\vdots \\
\vdots\end{array}: \vdots:$} & \multirow{2}{*}{$\begin{array}{llll}: & & \bullet \\
\vdots & & \\
\vdots & \bullet & \\
& & \\
\end{array}$} & & \multirow[t]{2}{*}{ - } & \multirow[t]{2}{*}{ : } & & $\begin{array}{c}\text { Upper } \\
\text { Miocene }\end{array}$ \\
\hline & & & & & & & & & & & 乌ั้ हัँ & $\begin{array}{c}\text { Lower } \\
\text { Pliocene }\end{array}$ \\
\hline $55-64.5$ & 2.8 & $\begin{array}{l}7-1,39-40 \\
7-1,43-44 \\
7-1,53-54 \\
7, \mathrm{CC} \\
\end{array}$ & \begin{tabular}{ll|} 
& $\bullet$ \\
$\vdots$ & \\
\end{tabular} & $\cdots \bullet$ & & & & & $\cdot$ &.$^{\bullet}$ & - & Upper Miocene \\
\hline $64.5-74$ & 4.1 & $\begin{array}{l}8-1,110-111 \\
8-2,61-62 \\
8-3,100-101 \\
8, \mathrm{CC}\end{array}$ &. & & & & & & $\cdot$ & • & & \\
\hline $74-83.5$ & 5.8 & $\begin{array}{l}9-1,110-111 \\
9-2,80-82 \\
9-2,119-121 \\
9-3,3-5 \\
9-3,81-83 \\
\end{array}$ & \begin{tabular}{ll|}
$\bullet$ & $\bullet$ \\
$\bullet$ & $\bullet$ \\
$\bullet$ & \\
\end{tabular} & & & & & & $\dot{0}$ & • & & \\
\hline & & $\begin{array}{l}9-3,103-105 \\
9-3,116-118 \\
9-3,136-138 \\
9-4,115-116 \\
9, \mathrm{CC} \\
\end{array}$ & \begin{tabular}{l|l} 
& \\
$\vdots$ & \\
$:$ & \\
\end{tabular} & & & & & & 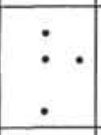 & : & & हूँ \\
\hline $83.5-93$ & 4.1 & $\begin{array}{l}10-1,56-58 \\
10-2,7-9 \\
10-3,80-82 \\
10, \mathrm{CC} \\
\end{array}$ & : & & & & & & & & ? & $\begin{array}{l}\frac{3}{2} \\
\frac{2}{2} \\
\frac{2}{2}\end{array}$ \\
\hline 93-102.5 & 3.3 & $\begin{array}{l}11-2,18-20 \\
11, \mathrm{CC}\end{array}$ & : & & & & & & & & & \\
\hline $102.5-112$ & 7.6 & $\begin{array}{l}12-1,90-92 \\
12-2,110-112 \\
12-4,40-42 \\
12-5,17-19 \\
12, \mathrm{CC} \\
\end{array}$ & $\begin{array}{l}\cdots \\
\bullet \cdot \\
\end{array}$ & & & & & & $\because$ & $\cdot$ & & \\
\hline $112-121.5$ & 5.3 & $\begin{array}{l}13-1,125-127 \\
13-2,5-7 \\
13-3,100-102 \\
13-4,120-122 \\
13, \mathrm{CC} \\
\end{array}$ & 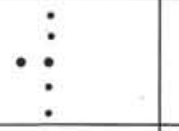 & & & & & & $\therefore$ & & & \\
\hline $121.5-131$ & 0.2 & $14, \mathrm{CC}$ & $\bullet$ & & & & & & $\cdots$ & $\cdot$ & & \\
\hline $131-140.5$ & 3.6 & $15-3,130-131$ & $\bullet \bullet$ & & & & & & - & & & \\
\hline
\end{tabular}


Planktonic Foraminifers and Other Microfossils at Hole 378, Core 1-11

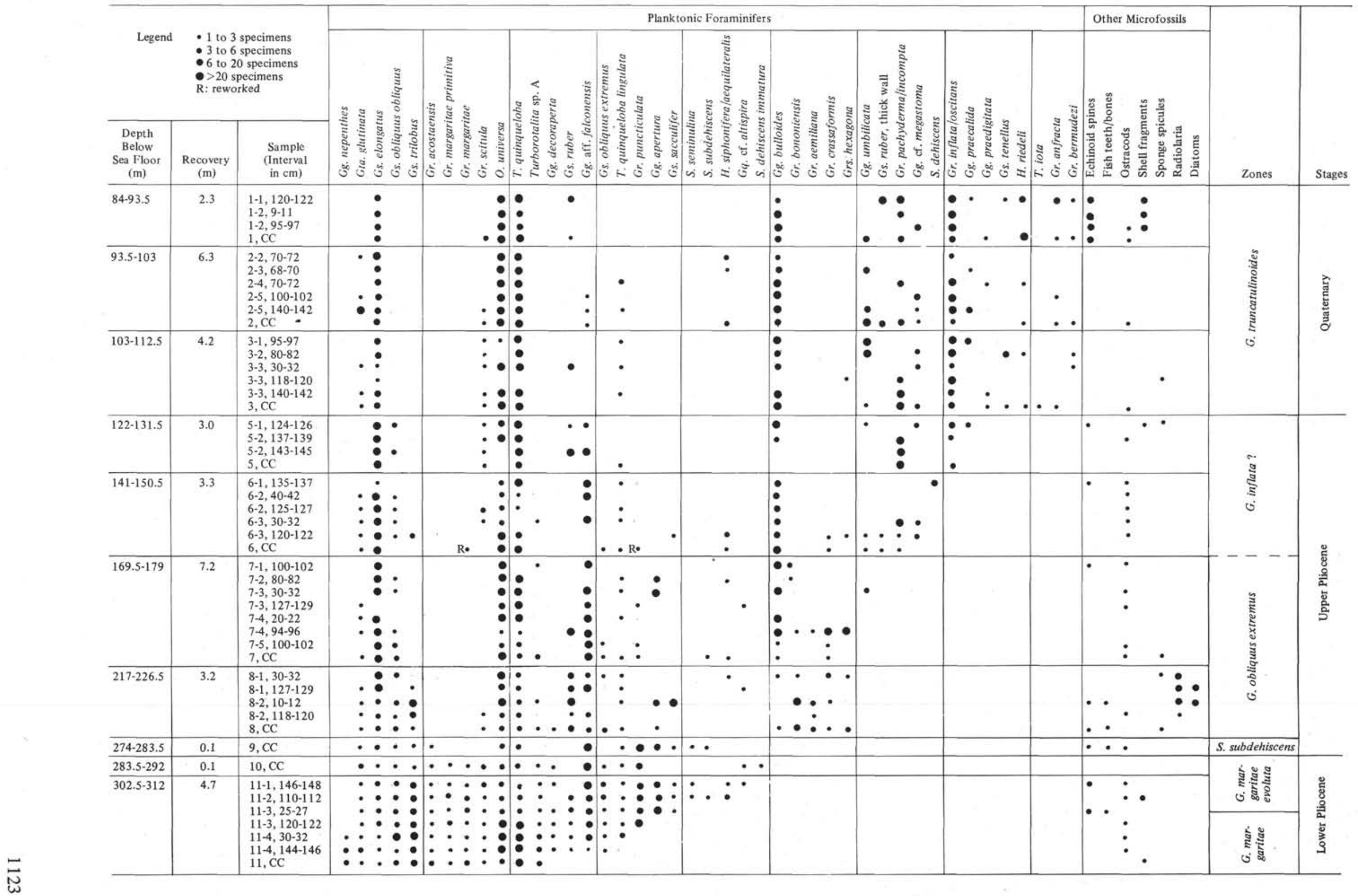


TABLE 24

Planktonic Foraminifers and Other Microfossils at Site 378A, Cores 1-4

\begin{tabular}{|c|c|c|c|c|c|c|c|c|c|c|c|c|c|c|}
\hline \multirow{2}{*}{\multicolumn{3}{|c|}{ Legend }} & \multicolumn{9}{|c|}{ Planktonic Foraminifers } & $\begin{array}{c}\text { Other } \\
\text { Microfossils }\end{array}$ & \multirow[b]{3}{*}{ Zones } & \multirow[b]{3}{*}{ Stages } \\
\hline & & & \multirow{2}{*}{ 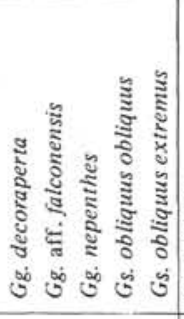 } & \multirow{2}{*}{ 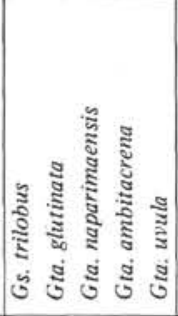 } & \multirow{2}{*}{ 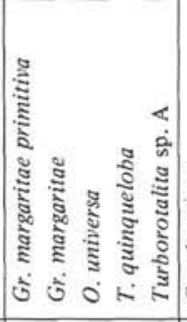 } & \multirow{2}{*}{ 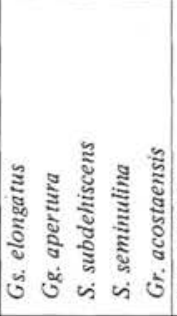 } & \multirow{2}{*}{ 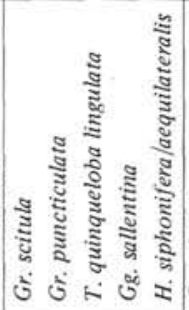 } & \multirow[b]{2}{*}{ 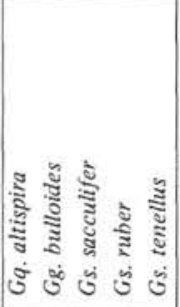 } & \multirow{2}{*}{ 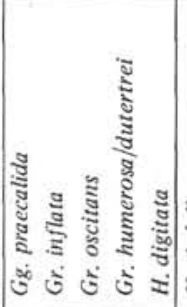 } & \multirow{2}{*}{ 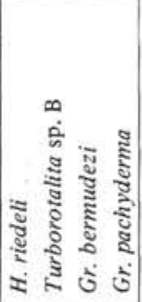 } & \multirow{2}{*}{ 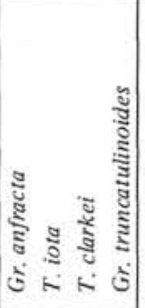 } & \multirow{2}{*}{ 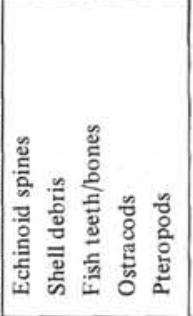 } & & \\
\hline $\begin{array}{c}\text { Depth } \\
\text { Below } \\
\text { Sea Floor } \\
(\mathrm{m}) \\
\end{array}$ & $\begin{array}{c}\text { Recovery } \\
(\mathrm{m})\end{array}$ & $\begin{array}{c}\text { Sample } \\
\text { (Interval } \\
\text { in } \mathrm{cm} \text { ) }\end{array}$ & & & & & & & & & & & & \\
\hline $46-55.5$ & 7.2 & $\begin{array}{l}1-1,130-132 \\
1-2,87-89 \\
1-3,120-122 \\
1-4,100-102 \\
1-5,60-62 \\
1, \mathrm{CC}\end{array}$ & & : & 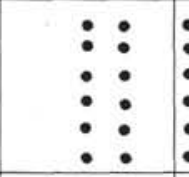 & : & $\begin{array}{ll}: & \\
: & . \\
0 & .\end{array}$ & $\begin{array}{lll}: & : & : \\
\vdots & \vdots & : \\
\vdots & \cdot & : \\
& \cdot & \end{array}$ & 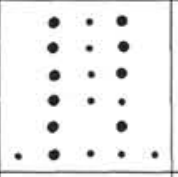 & 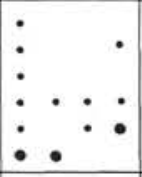 & 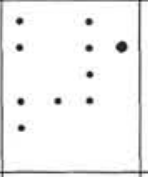 & $\begin{array}{lll}: & : & \bullet \\
: & : & \\
: & : & \cdot \\
& : & \\
\end{array}$ & 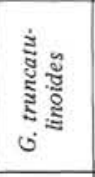 & 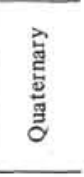 \\
\hline \multirow[t]{3}{*}{ 293-302.5 } & 9.5 & $\begin{array}{l}3-0,112-114 \\
3-0,140-142 \\
3-1,7-9 \\
3-1,100-102 \\
3-2,90-92 \\
3-3,4-6 \\
3-3,90-92 \\
\end{array}$ & $\begin{array}{ll}: & \vdots \\
\vdots & \vdots \\
\vdots & \vdots \\
: & :\end{array}$ & 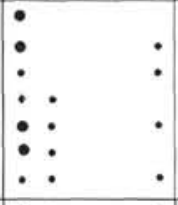 & 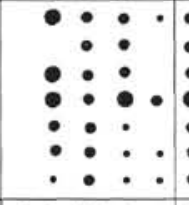 & 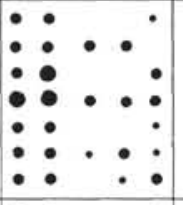 & 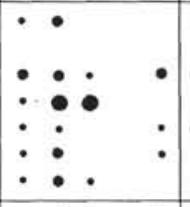 & $\begin{array}{l}\cdot \\
\cdot\end{array}$ & & & & $\begin{array}{l}: \div: \\
\therefore:\end{array}$ & \multirow{2}{*}{ 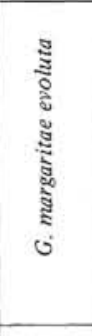 } & \multirow{4}{*}{ 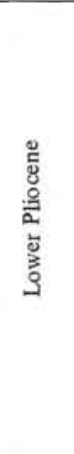 } \\
\hline & & $\begin{array}{l}3-4,100-102 \\
3-5,60-62 \\
3-6,30-32 \\
3-6,120-122 \\
3-6,134-136\end{array}$ & 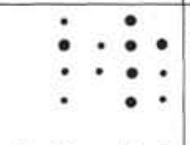 & $\because:$ & $\begin{array}{lllll}\bullet & \vdots & \bullet & \bullet & : \\
: & \vdots & \vdots & \vdots & : \\
\bullet & \bullet & \bullet & \vdots & \vdots\end{array}$ & 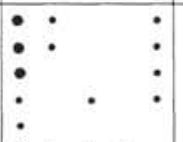 & $\begin{array}{lll}\bullet: & \bullet & \bullet \\
: & \bullet & \bullet\end{array}$ & & & & & $\dot{:}: \dot{:}$ & & \\
\hline & & $\begin{array}{l}3-6,148-150 \\
3, \mathrm{CC}\end{array}$ & $: \vdots: \vdots$ & $:: \dot{*}:$ & : $: \dot{\bullet}:$ & $: \cdot \cdot \dot{*}$ & & & & & & $\cdot \quad:$ & \multirow{2}{*}{ है ญั } & \\
\hline $302.5-312$ & 1.5 & $\begin{array}{l}4-1,1-2 \\
4-1,4-5\end{array}$ & $\vdots: \vdots \dot{*}$ & $\because: \vdots$. & $: \vdots: \vdots:$ & $\cdot \cdots \cdot$ & c. & & & & & $: \quad:$ & & \\
\hline
\end{tabular}


TABLE 25

Biostratigraphic Distribution of Benthic Foraminifers at Site 371

\begin{tabular}{|c|c|c|c|c|c|c|}
\hline Epoch & Quaternary & & te Plioce & & Early Pli & cene \\
\hline Stage/Zone & & M-PI 6 & M-PI S & M-Pl 4 & M-Pl 2-3 & M-PI 2 \\
\hline $\begin{array}{ll} & \text { Sample } \\
\text { Species } & \end{array}$ & 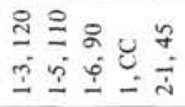 & i & m & 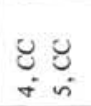 & $\begin{array}{ll}0 & 0 \\
0 & 0\end{array}$ & $\frac{N}{\infty}$ \\
\hline $\begin{array}{l}\text { Anomalinoides badensis } \\
\text { A. pseudogrosserugosa } \\
\text { Articulina tubulosa } \\
\text { Astrononion umbilicatulum } \\
\text { Bolivina ala ta }\end{array}$ & $\bullet \bullet \bullet \bullet \bullet$ & & $\bullet$ & $\bullet$ & & \\
\hline $\begin{array}{l}\text { B. arta } \\
\text { B. catanensis } \\
\text { B. compacta } \\
\text { B. dilitata } \\
\text { B. pseudoplicata } \\
\end{array}$ & $\bullet$ & & $\begin{array}{l}\bullet \\
\bullet \\
\bullet \\
\bullet\end{array}$ & $\bullet$ & & $\bullet$ \\
\hline $\begin{array}{l}\text { B. spathulata } \\
\text { B. subaenariensis } \\
\text { Bulimina aculeata } \\
\text { B. alazanensis } \\
\text { B. inflata } \\
\end{array}$ & $\bullet$ & $\bullet$ & $\begin{array}{l} \\
\bullet \\
\bullet\end{array}$ & & & \\
\hline $\begin{array}{l}\text { Cassidulina crassa } \\
\text { C. crassa, f. minima } \\
\text { C. laevigata } \\
\text { C. minuta } \\
\text { C. subglobosa } \\
\end{array}$ & $\bullet \bullet$ & - & : & • & & $\bullet$ \\
\hline $\begin{array}{l}\text { Chilostomella oolina } \\
\text { Cibicides lobatulus } \\
\text { C. pachyderma } \\
\text { C. wuellerstorfi } \\
\text { Cibicidoides bradyi } \\
\end{array}$ & $\begin{array}{lll}\bullet & & \\
& \bullet & \bullet \\
& \bullet\end{array}$ & • & • & - & $\bullet$ & \\
\hline $\begin{array}{l}\text { C. kullenbergi } \\
\text { C. robertsonianus } \\
\text { Discorbis aff. D. obtusa } \\
\text { D. parkerae } \\
\text { D. rosacea } \\
\end{array}$ & - & & $\begin{array}{l}\bullet \cdot \\
\bullet\end{array}$ & & $\bullet$ & $\bullet$ \\
\hline $\begin{array}{l}\text { Eggerella bradyi } \\
\text { Elphidium spp. } \\
\text { Epistominella exigua } \\
\text { Eponides pusillus } \\
\text { E. schreibersii } \\
\end{array}$ & $\bullet$ & $\bullet$ & $\begin{array}{l}\bullet \\
\bullet \\
\bullet\end{array}$ & $\bullet$ & $\bullet$ & $\bullet$ \\
\hline $\begin{array}{l}\text { Fursenkoina complanata } \\
\text { (ilobobulimina affinis } \\
\text { G. spp. } \\
\text { Guttulina spp. } \\
\text { Gyroidina laevigata } \\
\end{array}$ & $\begin{array}{lll}\bullet & & \\
\bullet & \bullet \\
& \bullet\end{array}$ & $\bullet$ & $\bullet$ & - & & \\
\hline $\begin{array}{l}\text { G. orbicularis } \\
\text { G. soldanii } \\
\text { G. umbonata } \\
\text { Karreriella bradyi } \\
\text { Lenticulina spp. } \\
\text { Loxostoma normale }\end{array}$ & $\bullet$ & $\bullet$ & : & • & & $\bullet$ \\
\hline $\begin{array}{l}\text { Melonis affine } \\
\text { M. barleeanum } \\
\text { M. soldanii } \\
\text { Neoconorbina terquemi } \\
\text { Nonion scaphum }\end{array}$ & $\bullet$ & & • & - & 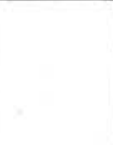 & \\
\hline $\begin{array}{l}\text { Nonionella sp. A } \\
\text { Nuttallides rugosus convexus } \\
\text { Oolininae } \\
\text { Oridorsalis umbonatus } \\
\text { Orthomorphina spp. } \\
\end{array}$ & $\bullet \bullet \bullet$ & $\bullet$ & : & - $\bullet$ & : & $\bullet$ \\
\hline $\begin{array}{l}\text { Pleurostomella alternans } \\
\text { Pullenia bulloides } \\
\text { Pyrgo depressa } \\
\text { P. elongata } \\
\text { Quinqueloculina lamarckiana } \\
\end{array}$ & • & & & & & \\
\hline $\begin{array}{l}\text { S. spp. } \\
\text { Robertina translucens } \\
\text { Sigmoilina tenuis } \\
\text { Sigmoilopsis schlumbergeri } \\
\text { Siphonina reticulata } \\
\end{array}$ & • & • & $\begin{array}{l} \\
\bullet \\
\end{array}$ & $\bullet$ & $\bullet$ & \\
\hline $\begin{array}{l}\text { Sphaeroidina bulloides } \\
\text { Stilstomella antillea } \\
\text { Trifarina angulosa pauperata } \\
\text { Valvulineria complanata }\end{array}$ & $\bullet$ & $\bullet$ & : & $\bullet$ & & \\
\hline
\end{tabular}


TABLE 26

Biostratigraphic Distribution of Benthic Foraminifers at Site 372

\begin{tabular}{|c|c|c|c|c|c|c|c|c|c|c|c|c|c|c|c|c|c|c|c|c|c|c|c|c|c|c|c|c|c|c|c|}
\hline Epoch & & Quat. & & & & te $\mathrm{Pl}$ & liocene & & ?E & Plio & vene & & & & & & & & & & Late $\mathrm{M}$ & Aiocer & & & & & & & & & \\
\hline Stage/Zone & & & & M-P & P1 6 & & M-Pl 5 & & & $1-\mathrm{PI} 3$ & & & & & & & Mes & sinian & & & & & & & & ortoni & & & & & \\
\hline Species & $\stackrel{9}{\Xi}$ & i & ن & $\frac{\dddot{2}}{\frac{i}{d}}$ & 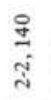 & $\begin{array}{l}\text { U } \\
\text { i }\end{array}$ & $\stackrel{\check{m}}{\vdots}$ & $\begin{array}{l}\text { के } \\
\text { ले }\end{array}$ & $\begin{array}{l}\tilde{b} \\
\dot{3} \\
\dot{n}\end{array}$ & $\underset{m}{U}$ & $\begin{array}{l}8 \\
\dot{8} \\
\dot{7}\end{array}$ & $\frac{i}{7}$ & $\stackrel{\infty}{\dddot{I}}$ & 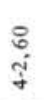 & $\begin{array}{l}\text { ฉ } \\
\text { กิ }\end{array}$ & $\begin{array}{l}\text { U } \\
\dot{0}\end{array}$ & 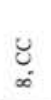 & $\stackrel{m}{\frac{m}{2}}$ & $\frac{\text { ㄱ. }}{\dot{3}}$ & $\frac{\text { I }}{\frac{y}{2}}$ & $\begin{array}{l}0 \\
\dot{\alpha}\end{array}$ & \begin{tabular}{l}
$\stackrel{\infty}{N}$ \\
\multirow{2}{*}{}
\end{tabular} & $\begin{array}{l}7 \\
\text { a }\end{array}$ & $\begin{array}{l}8 \\
i \\
2\end{array}$ & $\hat{\alpha}$ & $\frac{8}{2}$ & $\frac{5}{2}$ & $\begin{array}{l}\text { న్ } \\
\text { م் }\end{array}$ & $\begin{array}{l}\frac{9}{4} \\
\text { ja }\end{array}$ & 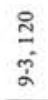 & $\frac{\text { I }}{\text { İ }}$ \\
\hline $\begin{array}{l}\text { Ammonia beccarii } \\
\text { Anomalina cicatricosa } \\
\text { Anomalinoides badensis } \\
\text { A. flinti } \\
\text { A. pseudogrosserugosa } \\
\end{array}$ & $\bullet$ & $\begin{array}{l}\bullet \\
\bullet \\
\end{array}$ & & $\bullet$ & : & & & & & & & & 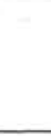 & & & & & $\bullet$ & $\bullet$ & $\bullet$ & - & $\bullet$ & • & & & & $\bullet$ & & $\bullet$ & & : \\
\hline $\begin{array}{l}\text { Articulina tubulosa } \\
\text { Asterigerinata mamilla } \\
\text { Astrononion umbilicatulum } \\
\text { Bigenerina nodosaria } \\
\text { Bolivina alata } \\
\end{array}$ & $\bullet$ & & - & & & & & $\bullet$ & : & $\bullet$ & $\bullet$ & & $\bullet$ & $\begin{array}{l}\cdot \\
\bullet\end{array}$ & $\bullet$ & $\begin{array}{l} \\
\bullet\end{array}$ & & & & & $\bullet$ & & & & & $\bullet$ & & & & & \\
\hline $\begin{array}{l}\text { B. albatrossi } \\
\text { B. arta } \\
\text { B. beyrichi } \\
\text { B. catanensis } \\
\text { B. compacta } \\
\end{array}$ & $\bullet$ & $\bullet$ & & $\bullet$ & $\bullet$ & & . & - & $\bullet$ & • & $\begin{array}{l}\bullet \\
\bullet \\
\end{array}$ & $\begin{array}{l}\bullet \\
\bullet \\
\end{array}$ & • & : & $\begin{array}{l}\bullet \\
\bullet \\
\end{array}$ & & & • & • & $\bullet$ & : & & - & & & • & & $\bullet$ & $\bullet$ & & • \\
\hline $\begin{array}{l}\text { B. dilitata } \\
\text { B. globulosa } \\
\text { B. hebes } \\
\text { B. incrassata } \\
\text { B. parvula } \\
\end{array}$ & & & & & $\bullet$ & & & & & : & & & $\begin{array}{l}\bullet \\
\bullet\end{array}$ & : & $\begin{array}{l}\bullet \\
\bullet \\
\end{array}$ & $\bullet$ & -5 & & : & & $\bullet$ & & & & & • & & & $\begin{array}{l}\bullet \\
\bullet\end{array}$ & & : \\
\hline $\begin{array}{l}\text { B. placentina } \\
\text { B. pseudoplicata } \\
\text { B. pseudopunctata } \\
\text { B. reticulata } \\
\text { B. silvestrina } \\
\end{array}$ & $\bullet$ & • & & & $\bullet$ & & & & $\bullet$ & $\begin{array}{l}\bullet \\
\bullet\end{array}$ & $\begin{array}{l}\bullet \\
\bullet\end{array}$ & $\bullet$ & $\begin{array}{l}\bullet \\
\bullet\end{array}$ & $\begin{array}{l}\bullet \\
\bullet\end{array}$ & $\begin{array}{l}\bullet \\
\bullet \\
\end{array}$ & : & 胥 & & : & & $\bullet$ & & & $\bullet$ & $\bullet$ & • & - & - & • & & \\
\hline $\begin{array}{l}\text { B. cf. B. simpsoni } \\
\text { B. spathulata } \\
\text { B. subaenariensis } \\
\text { B. sublobata } \\
\text { B. subspinescens } \\
\end{array}$ & & $\bullet$ & & & & & & & & $\bullet$ & : & $\bullet$ & $\bullet$ & : & : & $\bullet$ & & - & & $\bullet$ & $\bullet$ & • & & & $\bullet$ & . & & 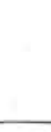 & $\bullet$ & & • \\
\hline $\begin{array}{l}\text { B. cf. B. thalmani } \\
\text { B. variabilis } \\
\text { Bolivinita concavomoenia } \\
\text { Bolivinopsis compta } \\
\text { Bulimina aculeata } \\
\end{array}$ & 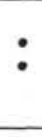 & $\bullet$ & - & & & & $\bullet$ & & & • & $\begin{array}{l}\bullet \\
\bullet \\
\end{array}$ & : & : & & & $\bullet$ & & - & & $\bullet$ & $\begin{array}{l}\bullet \\
\bullet\end{array}$ & $\bullet$ & • & $\begin{array}{l}\bullet \\
\bullet\end{array}$ & $\begin{array}{l}\bullet \\
\bullet\end{array}$ & 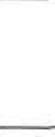 & $\bullet$ & $\begin{array}{l}\bullet \\
\bullet \\
\end{array}$ & • & $\bullet$ & : \\
\hline $\begin{array}{l}\text { B. affecta } \\
\text { B. alazanensis } \\
\text { B. barbata } \\
\text { B. buchiana } \\
\text { B. costata } \\
\end{array}$ & & & & & & & $\bullet$ & & $\bullet$ & $\bullet$ & $\bullet$ & $\bullet$ & $\bullet$ & & & & & & & • & & & & $\bullet$ & $\begin{array}{l}\bullet \\
\bullet\end{array}$ & $\bullet$ & : & $\bullet$ & & $\begin{array}{l}\bullet \\
\bullet\end{array}$ & \\
\hline $\begin{array}{l}\text { B. gibba } \\
\text { B. inflata } \\
\text { B. marginata } \\
\text { B. semicostata } \\
\text { Cassidulina crassa } \\
\end{array}$ & & $\bullet$ & • & & & & & & • & 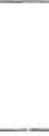 & : & $\bullet$ & • & & & $\bullet$ & & • & • & $\bullet$ & $\bullet$ & • & $\begin{array}{l}\bullet \\
\bullet \\
\end{array}$ & $\bullet$ & $\begin{array}{l}\bullet \\
\bullet\end{array}$ & $\bullet$ & $\bullet$ & $\bullet$ & $\bullet$ & $\begin{array}{l}\bullet \\
\bullet\end{array}$ & $\bullet$ \\
\hline $\begin{array}{l}\text { C. crassa, f. minima } \\
\text { C. braziliensis } \\
\text { C. Iaevigata } \\
\text { C. minuta } \\
\text { C. subglobosa } \\
\end{array}$ & & : & & : & $\begin{array}{l}\bullet \\
\bullet\end{array}$ & & : & • & & $\begin{array}{l}\bullet \\
\bullet \\
\bullet\end{array}$ & $\bullet$ & $:$ & $\begin{array}{l}\bullet \\
: \\
\end{array}$ & : & : & : & & & $\begin{array}{l}\bullet \\
\bullet \\
\bullet\end{array}$ & & : & & & & & : & • & & $\begin{array}{l}\bullet \\
: \\
\end{array}$ & & : \\
\hline $\begin{array}{l}\text { Chilostomella oolina } \\
\text { Cibicides akneriamus } \\
\text { C. austriacus } \\
\text { C. lobatulus } \\
\text { C. pachydermus } \\
\end{array}$ & & & $\bullet$ & $\bullet$ & $\bullet$ & & : & : & - & & $\bullet$ & & & $\bullet$ & • & $\bullet$ & & & & & & & & : & & 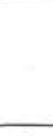 & & & • & & • \\
\hline $\begin{array}{l}\text { C. wuellerstorfi } \\
\text { C. spp. } \\
\text { Cibicidoides bradyi } \\
\text { C. kullenbergi } \\
\text { C. cf. C. kullenbergi }\end{array}$ & - & • & & $\begin{array}{l}\bullet \\
\bullet\end{array}$ & $\begin{array}{l}\bullet \\
\bullet\end{array}$ & & : & : & $\bullet$ & • & $\ddot{\bullet}:$ & $\bullet$ & $\bullet$ & : & $\bullet$ & & & & $\bullet$ & & : & 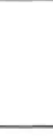 & t & $\bullet$ & : & : & $\begin{array}{l}\bullet \\
\bullet\end{array}$ & $\bullet$ & $\begin{array}{l}\bullet \\
\bullet \\
\bullet\end{array}$ & & : \\
\hline $\begin{array}{l}\text { C. robertsoniamus } \\
\text { Dentalina communis } \\
\text { Discorbis aff. D. columbiensis } \\
\text { D. globularis } \\
D \text {. aff. D. obtusa } \\
\end{array}$ & & & & & & & - & • & $\bullet$ & & & & • & & $\begin{array}{l}\bullet \\
\bullet \\
\end{array}$ & $\bullet$ & & & & & & & & & & & $\bullet$ & & : & & - \\
\hline $\begin{array}{l}\text { D. parkerae } \\
\text { D. rosacea } \\
\text { Eggerella bradyi } \\
\text { Ehrenbergina carinata } \\
\text { Elphidium spp. } \\
\end{array}$ & & $\begin{array}{l}\bullet \\
\bullet \\
\end{array}$ & $\bullet$ & & $\begin{array}{l}\bullet \\
\bullet\end{array}$ & & • & & & $\bullet$ & & & $\begin{array}{l} \\
\bullet \\
\end{array}$ & & • & & & & $\bullet$ & & & & & $\bullet$ & & & & & & & • \\
\hline $\begin{array}{l}\text { Epistominella exigua } \\
\text { Eponides polius } \\
\text { E. pusillus } \\
\text { E. schreibersii } \\
\text { Fursenkoina complanata } \\
\end{array}$ & & $\bullet$ & & $\bullet$ & $\bullet$ & - & - & 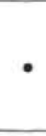 & $\bullet$ & $\begin{array}{l}\bullet \\
\vdots \\
\end{array}$ & $\bullet$ & • & $\begin{array}{l}\bullet \\
\bullet\end{array}$ & & $\begin{array}{l}\bullet \\
\bullet\end{array}$ & $\bullet$ & & & $\begin{array}{l}\bullet \\
\bullet \\
\bullet \\
\end{array}$ & & • & & & & • & $\bullet$ & & & • & & • \\
\hline $\begin{array}{l}\text { F. spp. } \\
\text { Gavelinopsis praegeri } \\
\text { Globobulimina affinis } \\
\text { G. aff. G. pseudospinescens } \\
\text { G. spp. }\end{array}$ & & $\bullet$ & & & & $\bullet$ & 1 & 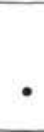 & $\bullet$ & $\bullet$ & & & • & & & $\bullet$ & & & & & • & & & & & & & & $\bullet$ & & - \\
\hline
\end{tabular}


TABLE 26 - Continued

\begin{tabular}{|c|c|c|c|c|c|c|c|c|c|c|c|c|c|c|c|c|c|c|c|c|c|c|c|c|c|c|c|c|c|c|}
\hline Epoch & & Quat. & & & & te $\mathrm{Pli}$ & iocene & & & Plio & cene & & & & & & & & & & ate Miocen & & & & & & & & & \\
\hline Stage/Zone & & & & M-P & PQ 6 & & M-PQ 5 & & & $\mathrm{P} \times 3$ & & & & & & & & ssinian & & & & & & & & rrtoni & & & & \\
\hline Species & $\stackrel{2}{\beth}$ & 官 & ¿ & $\frac{\dddot{q}}{\dot{d}}$ & $\frac{\mathscr{Z}}{\dot{1}}$ & $\begin{array}{l}\text { i } \\
\text { i }\end{array}$ & $\frac{9}{ \pm}$ & $\begin{array}{l}\text { \&े } \\
\text { กิ }\end{array}$ & $\begin{array}{l}\text { है } \\
\text { mें }\end{array}$ & i & $\begin{array}{l}8 \\
\frac{1}{4}\end{array}$ & $\frac{?}{i}$ & $\stackrel{\infty}{\stackrel{\sim}{]}}$ & $\begin{array}{l}\text { I } \\
8 \\
8\end{array}$ & $\begin{array}{l}\text { \& } \\
\text { İ }\end{array}$ & 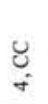 & $\underset{\infty}{0}$ & $\stackrel{m}{\vdots}$ & 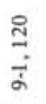 & 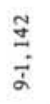 & 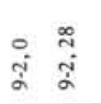 & $\begin{array}{l}\overline{7} \\
\text { ส }\end{array}$ & $\begin{array}{l}8 \\
\text { i் } \\
\text { i }\end{array}$ & ई̃ & $\begin{array}{l}\frac{8}{1} \\
\text { నi }\end{array}$ & $\frac{\pi}{2}$ & ஷ̊ & 官 & $\frac{\text { ஸे }}{2}$ & 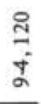 \\
\hline $\begin{array}{l}\text { Guttulina spp. } \\
\text { Gyroidina altiformis } \\
\text { G. delicata } \\
\text { G. laevigata } \\
\text { G. lamarckiana } \\
\end{array}$ & & & - & : & • & & & & $\bullet$ & $\bullet$ & $\bullet$ & & • & • & $\bullet$ & $\bullet$ & & & & & & & & & & & & • & & • \\
\hline $\begin{array}{l}\text { G. orbicularis } \\
\text { G. soldanii } \\
\text { G. umbonata } \\
\text { Hanzawaia boueana } \\
\text { Hyalinea balthica } \\
\end{array}$ & $\bullet$ & $\bullet$ & $\bullet$ & & & $\begin{array}{l}\bullet \\
\bullet\end{array}$ & $\bullet$ & • & & : & • & : & : & : & • & $:$ & & & - & & $\bullet$ & & $\bullet$ & $\bullet$ & $\begin{array}{l}\bullet \\
\bullet\end{array}$ & • & • & - & & $\begin{array}{l}\bullet \\
\bullet\end{array}$ \\
\hline $\begin{array}{l}\text { Karreriella bradyi } \\
\text { Lagenonodosaria scalaris } \\
\text { Lenticulina spp. } \\
\text { Loxostoma nomale } \\
\text { Martinottiella communis } \\
\end{array}$ & & $\bullet$ & : & $\bullet$ & $\begin{array}{l} \\
\bullet \\
\bullet\end{array}$ & - & • & & $\bullet$ & & $\bullet$ & $\bullet$ & : & & $\bullet$ & & & & $\bullet$ & & • & & - & & • & $\bullet$ & & $\bullet$ & & : \\
\hline $\begin{array}{l}\text { Melonis affine } \\
M \text {. barleeanum } \\
\text { M. soldanii } \\
\text { Neobulimina sp. } \\
\text { Neoconorbina terquemi } \\
\end{array}$ & & $\bullet$ & $\bullet$ & & $\bullet$ & : & : & : & - & $\bullet$ & : & - & $\bullet$ & $\bullet$ & • & $\bullet$ & & & & & & & & : & - & • & $\bullet$ & & & : \\
\hline $\begin{array}{l}\text { Nodosaria spp. } \\
\text { Nonion depressulus } \\
\text { N. scaphum } \\
\text { Nonionella sp. A } \\
\text { Nuttallides nugosus convexus } \\
\end{array}$ & $\bullet$ & & & & & & $\bullet$ & $\bullet$ & $\bullet$ & $\bullet$ & & $\bullet$ & & & $\bullet$ & & & & - & & • & & • & & $\bullet$ & & & $\bullet$ & & $\bullet$ \\
\hline $\begin{array}{l}\text { N. umbonifenis } \\
\text { Oolininae } \\
\text { Oridorsalis umbonatus } \\
\text { Orthomorphina exilis } \\
\text { O. pupoides } \\
\end{array}$ & $\bullet$ & : & & • & : & & : & : & • & : & : & : & • & $\because$ & $\begin{array}{l} \\
\bullet \\
\bullet\end{array}$ & $\bullet$ & & • & - & & • & & $\bullet$ & $\bullet$ & • & $\bullet$ & & • & & : \\
\hline $\begin{array}{l}\text { O. raphana } \\
\text { O. sp. } \\
\text { Osangularia cultur } \\
\text { Planulina ariminensis } \\
\text { Pleurostomella acuminata }\end{array}$ & $\bullet$ & & $\bullet$ & $\bullet$ & & $\bullet$ & & & • & & $\begin{array}{l} \\
\bullet\end{array}$ & • & - & - & • & $\bullet$ & & & $\bullet$ & & $\bullet$ & & $\bullet$ & $\bullet$ & $\bullet$ & $\bullet$ & & $\begin{array}{l}\bullet \\
\bullet\end{array}$ & & • \\
\hline $\begin{array}{l}\text { P. alternans } \\
\text { P. bierigi } \\
\text { P. spp. } \\
\text { Pseudociavulina crustata } \\
\text { Pullenia bulloides } \\
\end{array}$ & & & & & & & & $\bullet$ & • & . & $\begin{array}{l}\bullet \\
\bullet\end{array}$ & - & : & $\bullet$ & • & & & & & & - & & $\bullet$ & • & $\begin{array}{l}\bullet \\
\bullet \\
\bullet\end{array}$ & $\bullet$ & & & & $\begin{array}{l}\bullet \\
\bullet \\
\bullet \\
\end{array}$ \\
\hline $\begin{array}{l}\text { P. multilobata } \\
\text { P. osloensis } \\
\text { P. quinqueloba } \\
\text { P. salisbury: } \\
\text { P. subcarinata } \\
\end{array}$ & & & & & $\bullet$ & & & & $\bullet$ & & • & & : & & : & & & $\bullet$ & & & & & : & $\bullet$ & • & & & • & & - \\
\hline $\begin{array}{l}\text { Pyrgo depressa } \\
\text { P. elongata } \\
\text { Quinqueloculina lamarckiana } \\
\text { Q. venusta } \\
\text { Q. spp. } \\
\end{array}$ & & $\bullet$ & $\bullet$ & $\bullet$ & • & & & : & $\bullet$ & & $\bullet$ & & & & & & & & & & & & & & & $\bullet$ & & & & $\bullet$ \\
\hline $\begin{array}{l}\text { Rectouvigerina royoi } \\
\text { Robertina translucens } \\
\text { Seabrookia earlandi } \\
\text { Sigmoilina distorta } \\
\text { Sigmoilopsis schlum bergeri }\end{array}$ & $\bullet$ & & $\bullet$ & $\bullet$ & • & & $\bullet$ & $\bullet$ & $\bullet$ & $\bullet$ & & & & & & & & & & & & & $\bullet$ & & & & & : & & \\
\hline $\begin{array}{l}\text { Sigmoilina tenuis } \\
\text { Siphonina reticulata } \\
\text { S. tubulosa } \\
\text { Sphaeroidina bulloides } \\
\text { Spiroloculina caniculata }\end{array}$ & : & & $\bullet$ & $\bullet$ & • & & & • & $\bullet$ & : & $\bullet$ & & $\bullet$ & & • & & & & • & & - & & & & $\bullet$ & & & $\bullet$ & & \\
\hline $\begin{array}{l}\text { Stilostomella cf. } S \text {. annulifera } \\
\text { S. antillea } \\
\text { Trifarina angulosa angulosa } \\
T \text {. angulosa pauperata } \\
T \text {. bradyi } \\
\end{array}$ & $\bullet$ & • & & $\bullet$ & & & & & $\bullet$ & & $\begin{array}{l}\dot{\bullet} \\
\dot{0} \\
\end{array}$ & : & $\dot{\bullet}$ & $\begin{array}{l}\bullet \\
\bullet\end{array}$ & • & $\bullet$ & & & - & & - & & $\bullet$ & & • & $\bullet$ & & • & & $\begin{array}{r}\bullet \\
\bullet\end{array}$ \\
\hline $\begin{array}{l}\text { Triloculina tricarinata } \\
\text { Uvigerina auberiana } \\
\text { U. barbatula } \\
U \text {. hispida } \\
\text { U. laevis } \\
\end{array}$ & & & $\bullet$ & • & & & & $\bullet$ & & & & & & • & & • & & & & & & & $\bullet$ & & • & $\bullet$ & - & & & \\
\hline $\begin{array}{l}\text { U. longistriata } \\
U . \text { mediterranea } \\
U . \text { mioindex } \\
U \text {. peregrina } \\
U . \text { peregrina dinupta }\end{array}$ & & & • & $\bullet$ & $\bullet$ & & & $\bullet$ & • & & & & $\bullet$ & & & & & & & & & & $\bullet$ & & - & & & & & \\
\hline $\begin{array}{l}\text { U. proboscidea } \\
\text { U. nutila } \\
\text { Valvulineria complanata } \\
\text { Vulvulina pennatula }\end{array}$ & & $\bullet$ & & & & & $\bullet$ & - & • & : & : & - & $\bullet$ & $\vdots$ & • & & & & $\bullet$ & & & & $\bullet$ & $\bullet$ & : & & & $\bullet$ & & • \\
\hline
\end{tabular}


TABLE 26 - Continued

\begin{tabular}{|c|c|c|c|c|c|c|c|c|c|c|c|c|c|c|c|c|c|c|c|c|c|c|c|c|c|c|c|c|c|c|c|}
\hline Epoch & Lat & $\mathrm{MiC}$ & cene & & & & & & & & & & & & fiddle & Mioc & cene & & & & & & & & & & & & & & \\
\hline Stage/Zone & Tor & tonia & & & & & & & & & & & & & Serr: & avalia & & & & & & & & & & & & & & & \\
\hline Species & & $\begin{array}{l}\stackrel{0}{\vdots} \\
\dot{0}\end{array}$ & 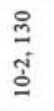 & $\begin{array}{l}8 \\
0\end{array}$ & $\begin{array}{l}\stackrel{9}{ \pm} \\
\vdots\end{array}$ & $\frac{0}{2}$ & $\begin{array}{l}\stackrel{8}{\infty} \\
\stackrel{+}{=} \\
=\end{array}$ & $\begin{array}{l}8 \\
=\end{array}$ & $\begin{array}{l}\infty \\
\infty \\
\stackrel{1}{\beth}\end{array}$ & 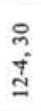 & $\begin{array}{l}\stackrel{0}{\Xi} \\
\stackrel{0}{\Xi}\end{array}$ & 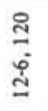 & $\begin{array}{l}8 \\
\text { I }\end{array}$ & $\begin{array}{l}\stackrel{\Xi}{ \pm} \\
\stackrel{1}{=}\end{array}$ & $\begin{array}{l}\text { ָे } \\
\text { ஸ் }\end{array}$ & $\begin{array}{l}0 \\
\dot{m} \\
\dot{m}\end{array}$ & $\begin{array}{l}\infty \\
\dot{1} \\
\stackrel{2}{-}\end{array}$ & $\stackrel{\stackrel{0}{-}}{\dot{n}}$ & $\begin{array}{l}\stackrel{ }{0} \\
\stackrel{0}{2}\end{array}$ & $\begin{array}{l}\dot{U} \\
\dot{n}\end{array}$ & 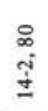 & 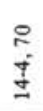 & $\frac{n}{ \pm}$ & $\begin{array}{l}8 \\
\pm\end{array}$ & $\begin{array}{l}0 \\
\stackrel{0}{-}\end{array}$ & $\begin{array}{l}\stackrel{1}{0} \\
\dot{0} \\
0\end{array}$ & $\begin{array}{l}0 \\
0 \\
0\end{array}$ & $\begin{array}{l}\stackrel{\Xi}{\vdots} \\
\vdots \\
\infty\end{array}$ & $\begin{array}{l}\mathcal{U} \\
\infty \\
\infty\end{array}$ & $\begin{array}{l}8 \\
0 \\
9\end{array}$ & $\frac{\text { q }}{\frac{\pi}{2}}$ \\
\hline $\begin{array}{l}\text { Ammonia beccarii } \\
\text { Anomalina cicatricosa } \\
\text { Anomalinoides badensis } \\
\text { A. flinti } \\
\text { A. pseudogrosserugosa } \\
\end{array}$ & & & & & $\bullet$ & $\vdots$ & & $\bullet$ & & & & $\bullet$ & & $\bullet$ & $\bullet$ & $\bullet$ & & • & : & $\bullet$ & & & $\begin{array}{l}\bullet \\
\bullet\end{array}$ & • & & $\bullet$ & & - & & & \\
\hline $\begin{array}{l}\text { Articulina tubulosa } \\
\text { Asterigerinata mamilla } \\
\text { Astrononion umbilicatulum } \\
\text { Bigenerina nodosaria } \\
\text { Bolivina alata } \\
\end{array}$ & & & $\begin{array}{l} \\
\bullet\end{array}$ & - & • & $\bullet$ & & • & $\bullet$ & & & $\bullet$ & & & $\bullet$ & & $\bullet$ & & $\bullet$ & & & & & & & $\bullet$ & & - & & & \\
\hline $\begin{array}{l}\text { B. albatrossi } \\
\text { B. arta } \\
\text { B. beyrichi } \\
\text { B. catanensis } \\
\text { B. compacta } \\
\end{array}$ & $\bullet$ & : & : & : & : & $:$ & : & & • & $\begin{array}{l}\cdot \\
\bullet \\
\end{array}$ & • & : & & $\begin{array}{l}\bullet \\
\vdots \\
\end{array}$ & : & : & $\bullet$ & $\begin{array}{l} \\
\bullet \\
\bullet\end{array}$ & • & & • & • & $\bullet$ & & & $\bullet$ & & & & & $\bullet$ \\
\hline $\begin{array}{l}\text { B. dilitata } \\
\text { B. globulosa } \\
\text { B. hebes } \\
\text { B. incrassata } \\
\text { B. panula } \\
\end{array}$ & & $\begin{array}{l}\bullet \\
\bullet\end{array}$ & : & . & & $\bullet$ & & & $\bullet$ & & & $\begin{array}{l}\bullet \\
\bullet \\
\end{array}$ & & • & $\bullet$ & • & $\bullet$ & $\bullet$ & $\bullet$ & & • & & & & & & & & & & $\bullet$ \\
\hline $\begin{array}{l}\text { B. placentina } \\
\text { B. pseudoplicata } \\
\text { B. pseudopunctata } \\
\text { B. reticulata } \\
\text { B. silvestrina } \\
\end{array}$ & & • & $\bullet$ & • & $\begin{array}{l}\bullet \\
\bullet\end{array}$ & • & : & & • & • & • & • & & • & • & • & & • & $\bullet$ & & : & • & $\begin{array}{l}\bullet \\
\bullet\end{array}$ & $\bullet$ & & $\begin{array}{l}\bullet \\
\bullet\end{array}$ & & $\bullet$ & & & • \\
\hline $\begin{array}{l}\text { B. cf. B. simpsoni } \\
\text { B. spathulata } \\
\text { B. subaenariensis } \\
\text { B. sublobata } \\
\text { B. subspinescens } \\
\end{array}$ & • & : & $\begin{array}{l}\bullet \\
\bullet \\
\bullet\end{array}$ & - & : & - & 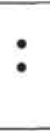 & $\bullet$ & : & & $\bullet$ & $\ddot{\bullet}$ & & • & $\because$ & & $\begin{array}{l}\dot{:} \\
:\end{array}$ & $\bullet$ & 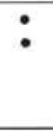 & & : & • & : & $\bullet$ & $\bullet$ & - & & $\bullet$ & & & \\
\hline $\begin{array}{l}\text { B. cf. B. thalmani } \\
\text { B. variabilis } \\
\text { Bolivinita concavomoenia } \\
\text { Bolivinopsis compta } \\
\text { Bulimine aculeata } \\
\end{array}$ & $\bullet$ & : & $\begin{array}{l}\bullet \\
\bullet\end{array}$ & : & $\bullet$ & : & : & : & $\bullet$ & $\bullet$ & & • & $\begin{array}{l} \\
\bullet \\
\bullet\end{array}$ & 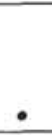 & • & & & $\bullet$ & $\bullet$ & & & & & & & $\begin{array}{l} \\
\bullet \\
\bullet\end{array}$ & $\bullet$ & & & & $\bullet$ \\
\hline $\begin{array}{l}\text { B. affecta } \\
\text { B. alezanensis } \\
\text { B. barbata } \\
\text { B. buchiana } \\
\text { B. costate } \\
\end{array}$ & & & $\begin{array}{l} \\
\bullet\end{array}$ & $\bullet$ & : & & $\bullet$ & $\bullet$ & & & & $\bullet$ & & & $\bullet$ & & & $\bullet$ & $\bullet$ & $\bullet$ & & $\bullet$ & & $\dot{\bullet}$ & & & & & & & \\
\hline $\begin{array}{l}\text { B. gibba } \\
\text { B. inflata } \\
\text { B. marginata } \\
\text { B. semicostata } \\
\text { Cassidulina crassa } \\
\end{array}$ & $\bullet$ & $\begin{array}{l}\bullet \\
\bullet\end{array}$ & $\bullet$ & $\bullet$ & $\bullet$ & & $\bullet$ & $\bullet$ & $\bullet$ & $\begin{array}{l}\bullet \\
\bullet\end{array}$ & & & • & & $\bullet$ & & & & $\bullet$ & & & & & $\bullet$ & & • & & $\bullet$ & & • & : \\
\hline $\begin{array}{l}\text { C. crassa. f. minima } \\
\text { C. braziliensis } \\
\text { C. laevigata } \\
\text { C. minuta } \\
\text { C. subglobosa } \\
\end{array}$ & & $\begin{array}{l}\bullet \\
\bullet \\
\bullet\end{array}$ & : & : & : & : & : & & $\begin{array}{l}\bullet \\
\bullet \\
\bullet\end{array}$ & $\begin{array}{l} \\
\\
\bullet\end{array}$ & $\begin{array}{l} \\
\\
\bullet\end{array}$ & $:$ & • & $\begin{array}{l} \\
: \\
\end{array}$ & $\begin{array}{l}\bullet \\
\bullet \\
\end{array}$ & $\begin{array}{l}\bullet \\
\vdots \\
\end{array}$ & • & & : & & : & $\begin{array}{l}\bullet \\
: \\
\bullet\end{array}$ & $\begin{array}{l}\bullet \\
: \\
\bullet\end{array}$ & $\bullet$ & & • & & $\begin{array}{l}\bullet \\
\bullet \\
\bullet\end{array}$ & & & : \\
\hline $\begin{array}{l}\text { Chilostomella oolina } \\
\text { Cibicides aknerianus } \\
\text { C. austriacus } \\
\text { C. lobatulus } \\
\text { C. pachydermus } \\
\end{array}$ & & • & $\bullet$ & $\bullet$ & $\bullet$ & - & $\bullet$ & • & • & • & $\bullet$ & • & • & $\bullet$ & & - & $\bullet$ & & & & : & • & : & • & & - & & $\bullet$ & & & • \\
\hline $\begin{array}{l}\text { C. wuellerstorfi } \\
\text { C. spp. } \\
\text { Cibicidoides bradyi } \\
\text { C. kullenbergi } \\
\text { C. cf. C. kullenbergi } \\
\end{array}$ & & & : & : & $\ddot{\bullet}$ & : & : & : & : & : & : & : & $\begin{array}{l}\bullet \\
\bullet\end{array}$ & : & : & : & : & : & $\ddot{\bullet}$ & : & : & : & • & $\begin{array}{l} \\
\bullet \\
\bullet\end{array}$ & & : & & $\begin{array}{l}: \\
\bullet \\
\bullet\end{array}$ & & & $\begin{array}{l}\dot{:} \\
\vdots \\
\end{array}$ \\
\hline $\begin{array}{l}\text {. robertsoniamus } \\
\text { Dentalina communis } \\
\text { Discorbis aff. } D \text {. columbiensis } \\
D . \text { globularis } \\
D \text {. aff. } D \text {. obtusa } \\
\end{array}$ & & & & & • & & & 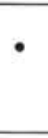 & & & & & & & & : & & & & & $\bullet$ & $\bullet$ & $\bullet$ & & & & & . & & & • \\
\hline $\begin{array}{l}\text { D. parkerae } \\
\text { D. rosacea } \\
\text { Eggerella bradyi } \\
\text { Ehrenbergina carinata } \\
\text { Elphidium spp. } \\
\end{array}$ & & & & & & & & - & & & & & 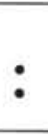 & & • & & & & & & & & & & & • & & & & & \\
\hline $\begin{array}{l}\text { Epistominella exigua } \\
\text { Eponides polius } \\
\text { E. pusillus } \\
\text { E. schreibersii } \\
\text { Fursenkoina complanate } \\
\end{array}$ & & : & $\begin{array}{l} \\
: \\
\end{array}$ & $\bullet$ & $\begin{array}{l}\bullet \\
\bullet\end{array}$ & $\begin{array}{l} \\
\bullet \\
\bullet\end{array}$ & $\begin{array}{l}\bullet \\
\bullet \\
\bullet\end{array}$ & & $\begin{array}{l} \\
\bullet \\
\bullet\end{array}$ & $\begin{array}{l} \\
\bullet \\
\bullet\end{array}$ & $\bullet$ & $\begin{array}{l}\bullet \\
\bullet \\
\bullet\end{array}$ & & $\begin{array}{l} \\
\bullet \\
\bullet\end{array}$ & $\begin{array}{l} \\
\bullet \\
\bullet\end{array}$ & - & $\begin{array}{l} \\
\bullet \\
\bullet\end{array}$ & $\begin{array}{l} \\
\bullet \\
-\end{array}$ & $\begin{array}{l}\cdot \\
\bullet \\
\bullet\end{array}$ & & & • & $\begin{array}{l}\bullet \\
\bullet\end{array}$ & & & • & & $\bullet$ & & & \\
\hline $\begin{array}{l}\text { F. spp. } \\
\text { Gavelinopsis praegeri } \\
\text { Globobulimina affinis } \\
\text { G. aff. G. pseudospinescens } \\
\text { G. spp. }\end{array}$ & & $\bullet$ & & & & & & & & & & & & & • & & & $\bullet$ & & & & & & & & • & $\bullet$ & & & & \\
\hline
\end{tabular}


TABLE 26 - Continued

\begin{tabular}{|c|c|c|c|c|c|c|c|c|c|c|c|c|c|c|c|c|c|c|c|c|c|c|c|c|c|c|c|c|c|c|c|}
\hline Epoch & & e Mic & cene & & & & & & & & & & & & fiddle & Mioc & cene & & & & & & & & & & & & & & \\
\hline Stage/Zone & & & & & & & & & & & & & & & Sert & avalia & & & & & & & & & & & & & & & \\
\hline$\underbrace{\text { Sample }}_{\text {Species }}$ & $\begin{array}{l}8 \\
0\end{array}$ & $\frac{0}{\vdots}$ & $\frac{\stackrel{\oplus}{-}}{\text { กิ }}$ & $\begin{array}{l}0 \\
0\end{array}$ & $\begin{array}{l}\stackrel{P}{ \pm} \\
\stackrel{Z}{=}\end{array}$ & $\begin{array}{l}\stackrel{\text { I }}{3} \\
\stackrel{3}{=}\end{array}$ & $\begin{array}{l}0 \\
\infty \\
\stackrel{1}{=}\end{array}$ & $\begin{array}{l}\text { U } \\
=\end{array}$ & $\begin{array}{l}\infty \\
\infty \\
\stackrel{1}{\simeq}\end{array}$ & 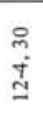 & $\begin{array}{l}\stackrel{0}{0} \\
\text { ป் }\end{array}$ & $\begin{array}{l}\stackrel{1}{\vdots} \\
\dot{0} \\
\simeq\end{array}$ & 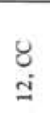 & $\begin{array}{l}\stackrel{0}{\Xi} \\
\dot{m}\end{array}$ & $\begin{array}{l}\text { ¿ } \\
\dot{1} \\
\dot{2}\end{array}$ & $\begin{array}{l}\infty \\
\infty \\
j \\
m \\
m\end{array}$ & $\begin{array}{l}8 \\
\dot{1} \\
2\end{array}$ & $\frac{\stackrel{\sim}{2}}{\tilde{m}}$ & $\begin{array}{l}\stackrel{9}{9} \\
\dot{q} \\
\stackrel{2}{2}\end{array}$ & $\begin{array}{l}\dot{8} \\
\text { 吾 }\end{array}$ & $\begin{array}{l}\text { I } \\
\text { i⿱ } \\
\infty\end{array}$ & $\begin{array}{l}\stackrel{R}{R} \\
\stackrel{+}{ \pm}\end{array}$ & $\begin{array}{l}\approx \\
\vdots \\
\dot{d}\end{array}$ & $\begin{array}{l}\dot{U} \\
\dot{\Xi}\end{array}$ & $\begin{array}{l}\dot{U} \\
\simeq\end{array}$ & $\begin{array}{l}\stackrel{ }{1} \\
\dot{0} \\
0\end{array}$ & $\begin{array}{l}\dot{U} \\
\stackrel{0}{0}\end{array}$ & 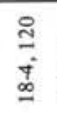 & $\begin{array}{l}8 \\
0 \\
\infty\end{array}$ & $\begin{array}{l}8 \\
0 \\
0\end{array}$ & 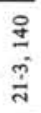 \\
\hline $\begin{array}{l}\text { Gutrulina spp. } \\
\text { Gyroidina altiformis } \\
\text { G. delicata } \\
\text { G. laevigata } \\
\text { G. lamarckiana }\end{array}$ & & & - & $\bullet$ & $\begin{array}{l}\bullet \\
\bullet\end{array}$ & & $\begin{array}{l}\bullet \\
\bullet\end{array}$ & $\bullet$ & $\bullet$ & & : & $\bullet$ & : & $\begin{array}{l}\bullet \\
\bullet\end{array}$ & : & $\bullet$ & $\bullet$ & • & : & & • & $\bullet$ & $\bullet$ & • & & : & & - & & & $\begin{array}{l}\bullet \\
\bullet\end{array}$ \\
\hline $\begin{array}{l}\text { G. orbicularis } \\
\text { G. soldanii } \\
\text { G. umbonata } \\
\text { Hanzawaia boueana } \\
\text { Hyalinea balthica } \\
\end{array}$ & $\bullet$ & • & $\begin{array}{l}\bullet \\
\bullet\end{array}$ & • & - & $\begin{array}{l}\bullet \\
\bullet\end{array}$ & • & $\begin{array}{l} \\
\bullet\end{array}$ & $\bullet$ & $\bullet$ & $\bullet$ & $\bullet$ & $\bullet$ & 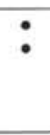 & $\bullet$ & $\begin{array}{l}\bullet \\
\bullet\end{array}$ & $\bullet$ & $\bullet$ & : & & $\begin{array}{l}\bullet \\
\bullet\end{array}$ & $\bullet$ & $\bullet$ & & & : & • & • & & & $\begin{array}{l}\bullet \\
\bullet \\
\bullet\end{array}$ \\
\hline $\begin{array}{l}\text { Karreriella bradyi } \\
\text { Lagenonodosaria scalaris } \\
\text { Lenticulina spp. } \\
\text { Loxostoma normale } \\
\text { Martinottiella communis }\end{array}$ & $\bullet$ & • & $\bullet$ & • & $\bullet$ & $\bullet$ & $\bullet$ & $\begin{array}{l}\bullet \\
\bullet\end{array}$ & $\bullet$ & : & : & - & $\begin{array}{l}\bullet \\
\bullet\end{array}$ & $\begin{array}{l}\bullet \\
\bullet\end{array}$ & $\begin{array}{l}\bullet \\
\bullet\end{array}$ & $\bullet$ & $\bullet$ & 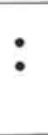 & • & • & $\begin{array}{l}\cdot \\
\bullet\end{array}$ & $\dot{\bullet}$ & • & $\begin{array}{l}\bullet \\
\bullet\end{array}$ & & : & $\bullet$ & • & & & $\cdot$ \\
\hline $\begin{array}{l}\text { Melonis affine } \\
\text { M. barleeanum } \\
\text { M. soldanii } \\
\text { Neobulimina sp. } \\
\text { Neoconorbina terquemi }\end{array}$ & • & & & : & • & $\begin{array}{l}\bullet \\
\bullet\end{array}$ & $\bullet$ & $\bullet$ & • & • & & $\bullet$ & $\bullet$ & - & & $\bullet$ & : & $\begin{array}{l}\bullet \\
\bullet\end{array}$ & $\bullet$ & • & & $\bullet$ & 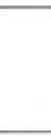 & & • & $\bullet$ & $\bullet$ & • & $\bullet$ & $\bullet$ & $\bullet$ \\
\hline $\begin{array}{l}\text { Nodosaria spp. } \\
\text { Nonion depressulus } \\
\text { N. scaphum } \\
\text { Nonionella sp. A. } \\
\text { Nuttallides rugosus convexus }\end{array}$ & & & & $\bullet$ & • & $\bullet$ & $\bullet$ & & $\bullet$ & $\bullet$ & : & $\begin{array}{l}\bullet \\
\bullet\end{array}$ & $\bullet$ & • & • & • & $\bullet$ & $\bullet$ & $\begin{array}{l}\cdot \\
\bullet\end{array}$ & $\bullet$ & • & - & $\begin{array}{l}\bullet \\
\bullet\end{array}$ & & & $\bullet$ & & 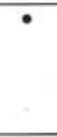 & & & $\bullet$ \\
\hline $\begin{array}{l}\text { N. umboniferus } \\
\text { Oolininae } \\
\text { Oridorsalis umbonatus } \\
\text { Orthomorphina exilis } \\
\text { O. pupoides } \\
\end{array}$ & $\bullet$ & : & : & • & $\dot{:}$ & : & $\ddot{\bullet}$ & • & 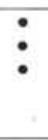 & 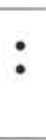 & $\dot{\bullet}$ & $\dot{\bullet}$ & 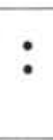 & $\dot{\bullet}$ & $\bullet$ & 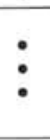 & $\dot{0}$ & $\dot{\bullet}$ & $\dot{0}$ & • & $\dot{\bullet}$ & : & : & & & : & & : & $\bullet$ & & $\dot{\bullet}$ \\
\hline $\begin{array}{l}\text { O. raphana } \\
\text { O. sp. } \\
\text { Osangularia cultur } \\
\text { Planulina ariminensis } \\
\text { Pleurostomella acuminata }\end{array}$ & • & : & $\begin{array}{l}\bullet \\
\bullet\end{array}$ & $\begin{array}{l}\bullet \\
\bullet\end{array}$ & • & $\bullet$ & $\begin{array}{l}\bullet \\
\bullet\end{array}$ & & $\begin{array}{l}\bullet \\
\bullet\end{array}$ & 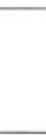 & $\bullet$ & $\begin{array}{l}\bullet \\
\bullet\end{array}$ & : & $\bullet$ & : & $\begin{array}{l}\bullet \\
\bullet\end{array}$ & $\begin{array}{l}\bullet \\
\bullet\end{array}$ & : & $\begin{array}{l}\bullet \\
\bullet\end{array}$ & & $\bullet$ & $\begin{array}{l}\bullet \\
\bullet\end{array}$ & $\begin{array}{l}\bullet \\
\bullet\end{array}$ & & & $\bullet$ & & $\bullet$ & & & $\bullet$ \\
\hline $\begin{array}{l}\text { P. alternans } \\
\text { P. bierigi } \\
\text { P. spp. } \\
\text { Pseudoclavulina crustata } \\
\text { Pullenia bulloides } \\
\end{array}$ & $\bullet$ & $\bullet$ & & • & $\bullet$ & $\begin{array}{l}\bullet \\
\bullet\end{array}$ & $\bullet$ & & : & $\bullet$ & & $\begin{array}{l}\bullet \\
\bullet\end{array}$ & • & : & : & : & & $\bullet$ & $\bullet$ & $\bullet$ & & • & - & $\bullet$ & $\bullet$ & $\bullet$ & $\bullet$ & $\bullet$ & & & 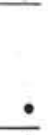 \\
\hline $\begin{array}{l}\text { P. multilobata } \\
\text { P. osloensis } \\
\text { P. quinqueloba } \\
\text { P. salisburyi } \\
\text { P. subcarinata } \\
\end{array}$ & & $\bullet$ & & $\begin{array}{l}\bullet \\
\bullet\end{array}$ & $\begin{array}{l}\bullet \\
\bullet\end{array}$ & $\begin{array}{l} \\
\bullet\end{array}$ & $\bullet$ & & & $\bullet$ & • & $\begin{array}{l}\bullet \\
\bullet\end{array}$ & & 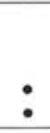 & $\begin{array}{l} \\
\bullet\end{array}$ & $\begin{array}{l}\bullet \\
\bullet \\
\bullet\end{array}$ & : & $\begin{array}{l}\bullet \\
\bullet\end{array}$ & $\begin{array}{l}\bullet \\
\bullet \\
\bullet\end{array}$ & • & $\begin{array}{l}\cdot \bullet \\
\bullet\end{array}$ & $\bullet$ & • & - & & • & & • & & & $\begin{array}{l}\cdot \\
\bullet\end{array}$ \\
\hline $\begin{array}{l}\text { Pyrgo depressa } \\
\text { P. elongata } \\
\text { Quinqueloculina lamarckiana } \\
\text { Q. venusta } \\
\text { Q. spp. } \\
\end{array}$ & $\bullet$ & & & & & & & & $\bullet$ & & & & & & $\bullet$ & $\bullet$ & • & & $\bullet$ & • & & & & & & $\bullet$ & $\bullet$ & & & & \\
\hline $\begin{array}{l}\text { Rectouvigerina royoi } \\
\text { Robertina translucens } \\
\text { Seabrookia earlandi } \\
\text { Sigmoilina distorta } \\
\text { Sigmoilopsis schlumbergeri }\end{array}$ & & & • & • & & $\bullet$ & • & • & $\begin{array}{l}\bullet \\
\bullet\end{array}$ & & & $\begin{array}{l}\bullet \\
\bullet\end{array}$ & • & $\bullet$ & $\begin{array}{l}\bullet \\
\bullet \\
\bullet\end{array}$ & & & $\begin{array}{l}\bullet \\
\bullet\end{array}$ & $\begin{array}{l}\bullet \\
\bullet\end{array}$ & • & $\bullet$ & & $\bullet$ & & $\bullet$ & $\bullet$ & $\bullet$ & $\bullet$ & $\bullet$ & & \\
\hline $\begin{array}{l}\text { Sigmoilina tenuis } \\
\text { Siphonina reticulata } \\
\text { S. tubulosa } \\
\text { Sphaeroidina bulloides } \\
\text { Spiroloculina caniculata }\end{array}$ & $\bullet$ & & & $\begin{array}{l}\bullet \\
\bullet\end{array}$ & & & : & $\bullet$ & : & & $\bullet$ & & $\begin{array}{l}\bullet \\
\bullet\end{array}$ & : & $\begin{array}{l}\bullet \\
\bullet\end{array}$ & $\begin{array}{l}\bullet \\
\bullet\end{array}$ & $\bullet$ & $\bullet$ & : & & & $\bullet$ & $\bullet$ & $\bullet$ & & • & • & & & & $\bullet$ \\
\hline $\begin{array}{l}\text { Stilostomella } \text { cf. S. annulifera } \\
\text { S. antillea } \\
\text { Trifarina angulosa angulosa } \\
\text { T. angulosa pauperata } \\
T \text {. bradyi } \\
\end{array}$ & & $\because$ & : & : & : & $\begin{array}{l}\dot{\bullet} \\
\bullet \\
\end{array}$ & : & $\bullet$ & : & $\bullet$ & $\begin{array}{l}\bullet \\
\bullet\end{array}$ & $\begin{array}{l}\bullet \\
\bullet \\
\bullet\end{array}$ & 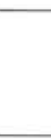 & $\begin{array}{l}\bullet \\
\bullet \\
\end{array}$ & $\begin{array}{l}\bullet \\
\bullet \\
\end{array}$ & $\begin{array}{l}\dot{\bullet} \\
\bullet \\
\end{array}$ & $\because$ & $\begin{array}{l}\bullet \\
\bullet \\
\end{array}$ & : & • & : & : & $\begin{array}{l}\dot{\bullet} \\
\bullet \\
\end{array}$ & • & & $\begin{array}{l}\bullet \\
\bullet \\
\bullet\end{array}$ & $\bullet$ & $\begin{array}{l}\bullet \\
\bullet \\
\end{array}$ & 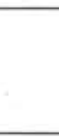 & & $\dot{\bullet}$ \\
\hline $\begin{array}{l}\text { Triloculina tricarinata } \\
\text { Uvigerina auberiana } \\
\text { U. barbatula } \\
\text { U. hispida } \\
\text { U. laevis } \\
\end{array}$ & $\bullet$ & $\bullet$ & & • & & & • & $\bullet$ & • & $\bullet$ & & $\bullet$ & • & • & & & & & $\bullet$ & $\bullet$ & • & & & & - & & • & & $\bullet$ & & - \\
\hline $\begin{array}{l}U . \text { longistriata } \\
U . \text { mediterranea } \\
U . \text { mioindex } \\
\text { U. peregrina } \\
U . \text { peregrina dirupta }\end{array}$ & & & & & & & & $\bullet$ & & & & & $\bullet$ & & & & & & & & & & & & & & & & & & \\
\hline $\begin{array}{l}\text { U. proboscidea } \\
\text { U. rutila } \\
\text { Valvulineria complanata } \\
\text { Vulvulina pennatula } \\
\end{array}$ & & & & • & & & & $\bullet$ & & & $\bullet$ & & : & • & & & - & & & & & 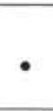 & ( & & & $\bullet$ & & & & & \\
\hline
\end{tabular}


TABLE 26 - Continued

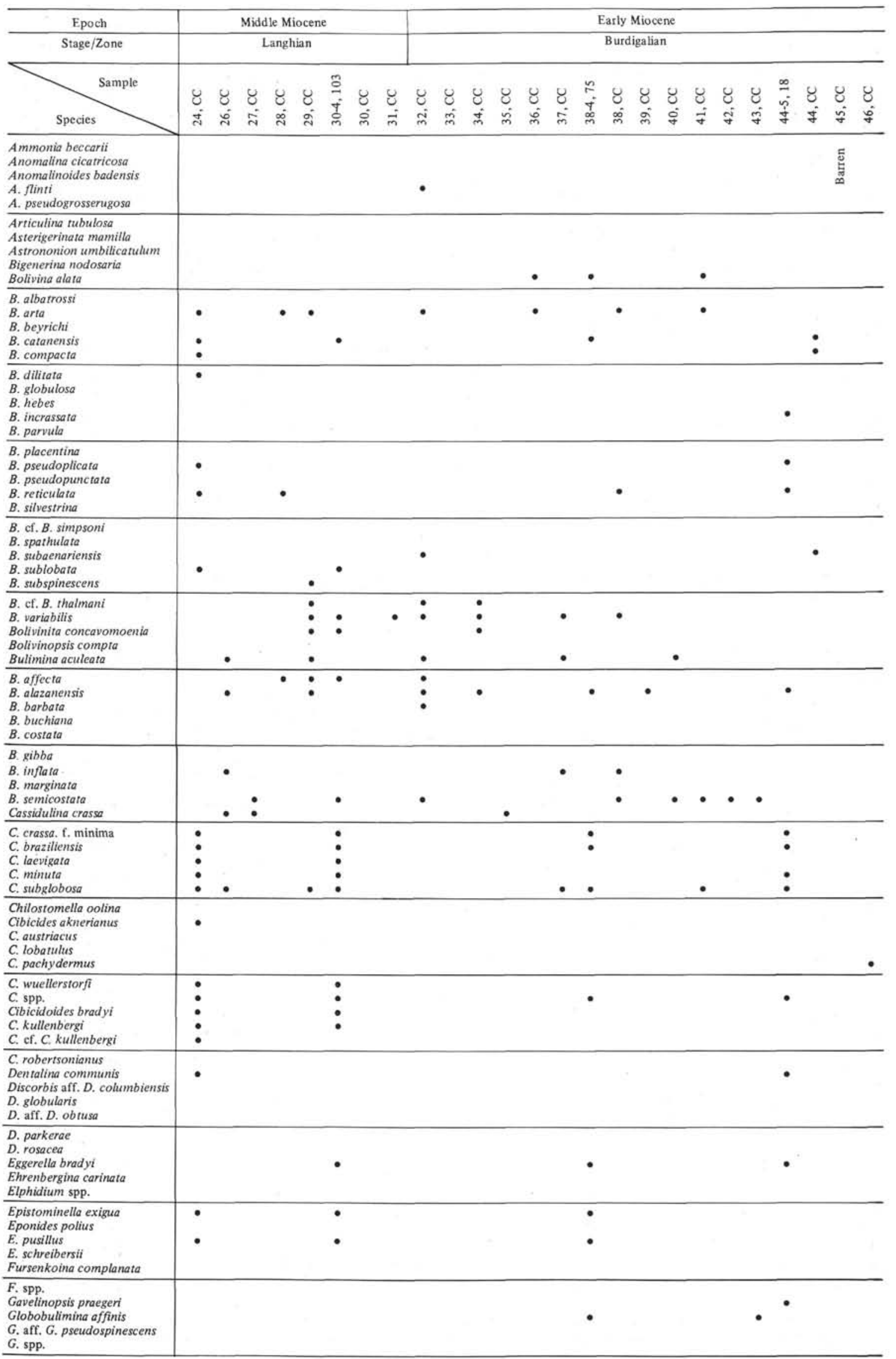


TABLE 26 - Continued

\begin{tabular}{|c|c|c|c|c|c|c|c|c|c|c|c|c|c|c|c|c|c|c|c|c|c|c|c|c|c|}
\hline Epoch & & & Mid & 1le M & iocen & & & & & & & & & & & arly $\mathrm{N}$ & Mioce & & & & & & & & \\
\hline Stage/Zone & & & & angh & & & & & & & & & & & & Burdi & igalia & & & & & & & & \\
\hline$\underbrace{\text { Sample }}_{\text {Species }}$ & $\begin{array}{l}8 \\
\text { J }\end{array}$ & & $\begin{array}{l}\tilde{U} \\
\text { ì }\end{array}$ & $\begin{array}{l}\mathcal{U} \\
\dot{\sim}\end{array}$ & $\begin{array}{l}\text { రิ } \\
\text { જે }\end{array}$ & $\frac{\text { @ }}{0}$ & $\begin{array}{l}\text { U } \\
\text { ¿ }\end{array}$ & $\begin{array}{l}\text { U } \\
\text { m }\end{array}$ & $\begin{array}{l}8 \\
\text { กิ }\end{array}$ & $\begin{array}{l}\text { U } \\
\text { ल }\end{array}$ & $\begin{array}{l}\text { ¿ } \\
\dot{f}\end{array}$ & $\begin{array}{l}y \\
\text { m. }\end{array}$ & $\begin{array}{l}8 \\
\hat{d}\end{array}$ & $\begin{array}{l}8 \\
\text { in }\end{array}$ & $\stackrel{n}{\stackrel{n}{\square}}$ & $\begin{array}{l}\text { U } \\
\infty \\
\infty\end{array}$ & $\begin{array}{l}8 \\
\text { g. }\end{array}$ & $\begin{array}{l}8 \\
\dot{8} \\
\dot{8}\end{array}$ & $\begin{array}{l}8 \\
-7\end{array}$ & $\begin{array}{l}\mathcal{U} \\
\mathcal{f}\end{array}$ & $\begin{array}{l}8 \\
\text { m }\end{array}$ & $\frac{\infty}{\stackrel{\infty}{\dot{q}}}$ & $\begin{array}{l}8 \\
\dot{8}\end{array}$ & $\begin{array}{l}8 \\
y\end{array}$ & $\begin{array}{l}8 \\
8 \\
0\end{array}$ \\
\hline $\begin{array}{l}\text { Guttulina spp. } \\
\text { Gyroidina altiformis } \\
\text { G. delicata } \\
\text { G. laevigata } \\
\text { G. lamarckiana }\end{array}$ & & & & & & $\bullet$ & & & & & & & & & $\vdots$ & & . & & & & & & & $=$ & \\
\hline $\begin{array}{l}\text { G. orbicularis } \\
\text { G. soldanii } \\
\text { G. umbonata } \\
\text { Hanzawaia boueana } \\
\text { Hyalinea balthica }\end{array}$ & - & • & • & • & & - & - & - & $\bullet$ & & $\bullet$ & - & - & & : & - & $\bullet$ & $\bullet$ & $\bullet$ & • & • & $\bullet$ & • & & $\bullet$ \\
\hline $\begin{array}{l}\text { Karreriella bradyi } \\
\text { Lagenonodosaria scalaris } \\
\text { Lenticulina spp. } \\
\text { Loxostoma normale } \\
\text { Martinottiella communis }\end{array}$ & $\bullet$ & $\bullet$ & & & & $\bullet$ & & & $\bullet$ & & & & - & & : & & & & & & $\bullet$ & $\bullet$ & & & \\
\hline $\begin{array}{l}\text { Melonis affine } \\
\text { M. barleeanum } \\
\text { M. soldanii } \\
\text { Neobulimina sp. } \\
\text { Neoconorbina terquemi }\end{array}$ & $\bullet$ & & & & $\bullet$ & • & • & & $\bullet$ & - & & • & & $\bullet$ & $\bullet$ & $\bullet$ & $\stackrel{\bullet}{\bullet}$ & : & $\bullet$ & & & : & & & \\
\hline $\begin{array}{l}\text { Nodosaria spp. } \\
\text { Nonion depressulus } \\
\text { N. scaphum } \\
\text { Nonionella sp. A } \\
\text { Nuttallides rugosus convexus }\end{array}$ & $\bullet$ & & & & & - & & & & & & & & & - & & & & & & & $\bullet$ & & & \\
\hline $\begin{array}{l}\text { N. umboniferus } \\
\text { Oolininae } \\
\text { Oridorsalis umbonatus } \\
\text { Orthomorphina exilis } \\
\text { O. pupoides } \\
\end{array}$ & : & • & : & & & : & & & & & & - & & & : & • & • & & $\bullet$ & $\bullet$ & & : & & & \\
\hline $\begin{array}{l}\text { O. raphana } \\
\text { O. sp. } \\
\text { Osangularia cultur } \\
\text { Planulina ariminensis } \\
\text { Pleurostomella acuminata }\end{array}$ & $\bullet$ & & & $\bullet$ & & : & & & & & & $\bullet$ & & & & & & & & & - & & & & \\
\hline $\begin{array}{l}\text { P. alternans } \\
\text { P. bierigi } \\
\text { P. spp. } \\
\text { Pseudoclavulina crustata } \\
\text { Pullenia bulloides }\end{array}$ & $\bullet$ & & $\bullet$ & & & • & - & & $\bullet$ & - & & $\bullet$ & $\bullet$ & & $\bullet$ & $\bullet$ & $\bullet$ & & - & $\bullet$ & • & & & & \\
\hline $\begin{array}{l}\text { P. multilobata } \\
\text { P. osloensis } \\
\text { P. quinqueloba } \\
\text { P. salisburyi } \\
\text { P. subcarinata } \\
\end{array}$ & • & & & & & : & & & & & & & $\bullet$ & & & & & & & & & & & & \\
\hline $\begin{array}{l}\text { Pyrgo depressa } \\
\text { P. elongata } \\
\text { Quinqueloculina lamarckiana } \\
\text { Q. venusta } \\
Q \text {. spp. }\end{array}$ & & & & & $\bullet$ & & & & & & & & & & & & & & & & $\bullet$ & & & & \\
\hline $\begin{array}{l}\text { Rectouvigerina royoi } \\
\text { Robertina translucens } \\
\text { Seabrookia earlandi } \\
\text { Sigmoilina distorta } \\
\text { Sigmoilopsis schlumbergeri }\end{array}$ & $\bullet$ & & & • & & $\bullet$ & & & & & & & & & & & & & & & & & & & \\
\hline $\begin{array}{l}\text { Sigmoilina tenuis } \\
\text { Siphonina reticulata } \\
\text { S. tubulosa } \\
\text { Sphaeroidina bulloides } \\
\text { Spiroloculina caniculata }\end{array}$ & • & - & & & & : & $\bullet$ & & $\bullet$ & $\bullet$ & & • & $\bullet$ & & : & & $\bullet$ & $\bullet$ & & $\bullet$ & $\bullet$ & $\bullet$ & & & \\
\hline $\begin{array}{l}\text { Stilostomella ef. } S \text {. annulifera } \\
\text { S. antillea } \\
\text { Trifarina angulosa angulosa } \\
\text { T. angulosa pauperata } \\
\text { T. bradyi }\end{array}$ & • & 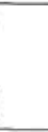 & • & & $\because$ & $\begin{array}{l}\bullet \\
:\end{array}$ & & & & & & & & & $\ddot{\bullet}$ & & & & & & & : & & & \\
\hline $\begin{array}{l}\text { Trilocutina tricarinata } \\
\text { Uvigerina auberiana } \\
U . \text { barbatula } \\
U . \text { hispida } \\
U \text {. laevis }\end{array}$ & & & & & & & & & & & & $\bullet$ & & - & - & • & & $\bullet$ & & - & & & & & \\
\hline $\begin{array}{l}\text { U. longistriata } \\
\text { U. mediterranea } \\
\text { U. mioindex } \\
\text { U. peregrina } \\
\text { U. peregrina dinupta }\end{array}$ & & & & & & & & & & & & - & & 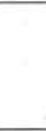 & & & & & & 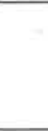 & & & & & \\
\hline $\begin{array}{l}\text { U. proboscidea } \\
\text { U. rutila } \\
\text { Valvulineria complanata } \\
\text { Vulvulina pennatula }\end{array}$ & 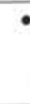 & & & $\bullet$ & & $\bullet$ & & & & & & & & & & & & - & & $\bullet$ & & & & & \\
\hline
\end{tabular}


TABLE 27

Biostratigraphic Distribution of Benthic Foraminifers at Hole 373A

\begin{tabular}{|c|c|c|}
\hline Epoch & Quaternary & Pliocene \\
\hline Stage/Zone & & M-PQ 3-6 \\
\hline Species & 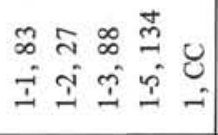 & 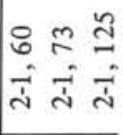 \\
\hline $\begin{array}{l}\text { Articulina tubulosa } \\
\text { Bigenerina nodosaria } \\
\text { Bolivina pseudoplicata } \\
\text { Bulimina aculeata } \\
\text { Cassidulina crassa } \\
\end{array}$ & 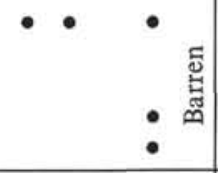 & $\bullet$ \\
\hline $\begin{array}{l}\text { C. laevigata } \\
\text { Eggerella bradyi } \\
\text { Eponides pusillus } \\
\text { Gyroidina laevigata } \\
\text { G. lararckiana } \\
\end{array}$ & - $\bullet$ & : \\
\hline $\begin{array}{l}\text { G. soldanii } \\
\text { Karreriella bradyi } \\
\text { Nonion scaphum } \\
\text { Olininae } \\
\text { Oridorsalis umbonatus } \\
\end{array}$ & $\bullet \bullet \bullet \bullet \bullet$ & $\begin{array}{l}\bullet \\
\bullet \\
\bullet \\
\bullet\end{array}$ \\
\hline $\begin{array}{l}\text { Pleurostomella alternans } \\
\text { Pullenia salisburyi } \\
\text { Pyrgo depressa } \\
\text { Quinqueloculina venusta } \\
\text { Q. spp. } \\
\end{array}$ & 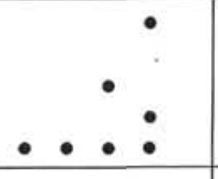 & - $\bullet$ \\
\hline $\begin{array}{l}\text { Siphonina reticulata } \\
\text { Spiroloculina caniculata } \\
\text { Triloculina tricarinata }\end{array}$ & $\bullet$ & $\bullet$ \\
\hline
\end{tabular}


TABLE 28

Biostratigraphic Distribution of Benthic Foraminifers at Site 374

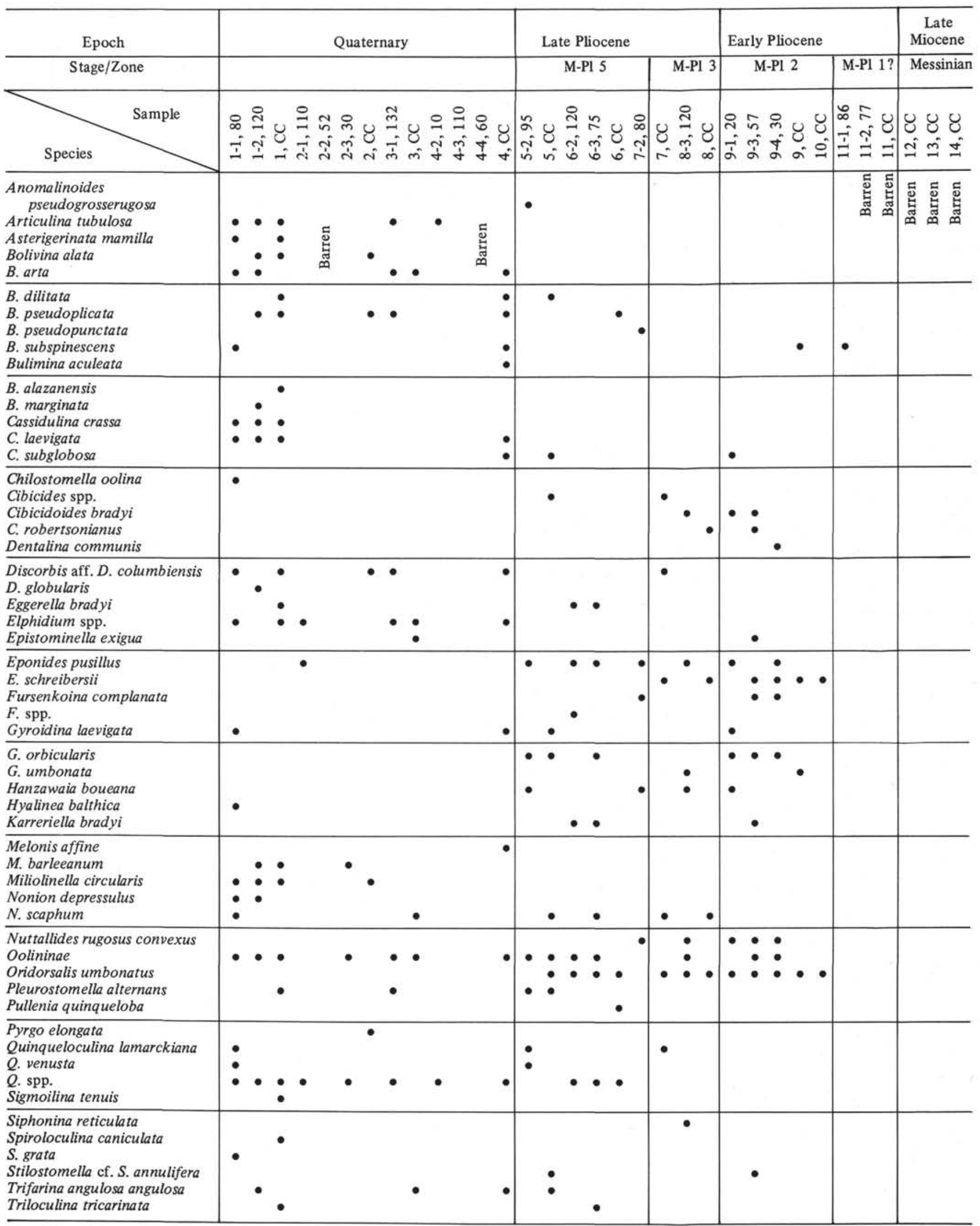


TABLE 29

Biostratigraphic Distribution of Benthic Foraminifers at Site 375

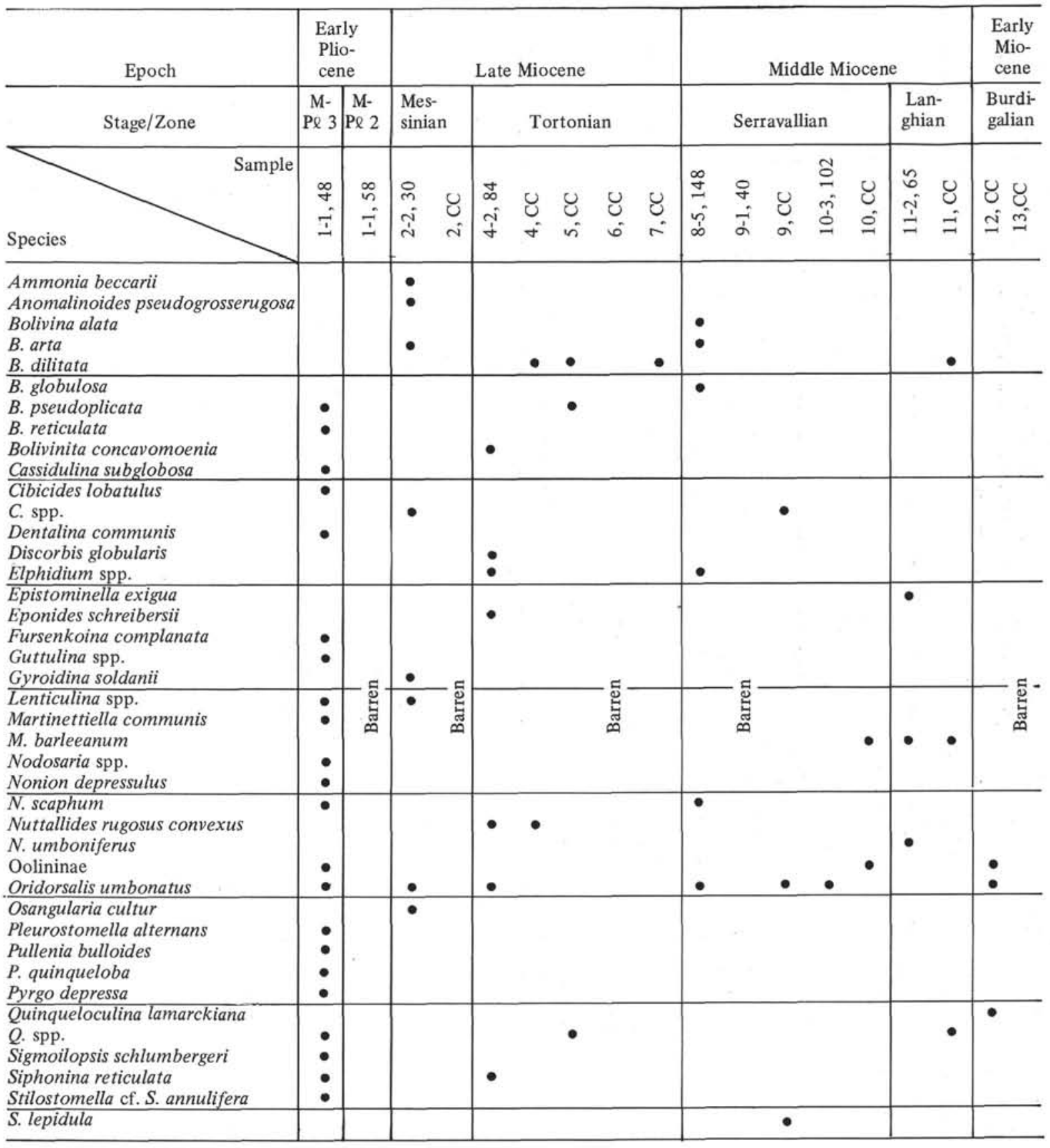


TABLE 30

Biostratigraphic Distribution of Benthic Foraminifers at Site 376

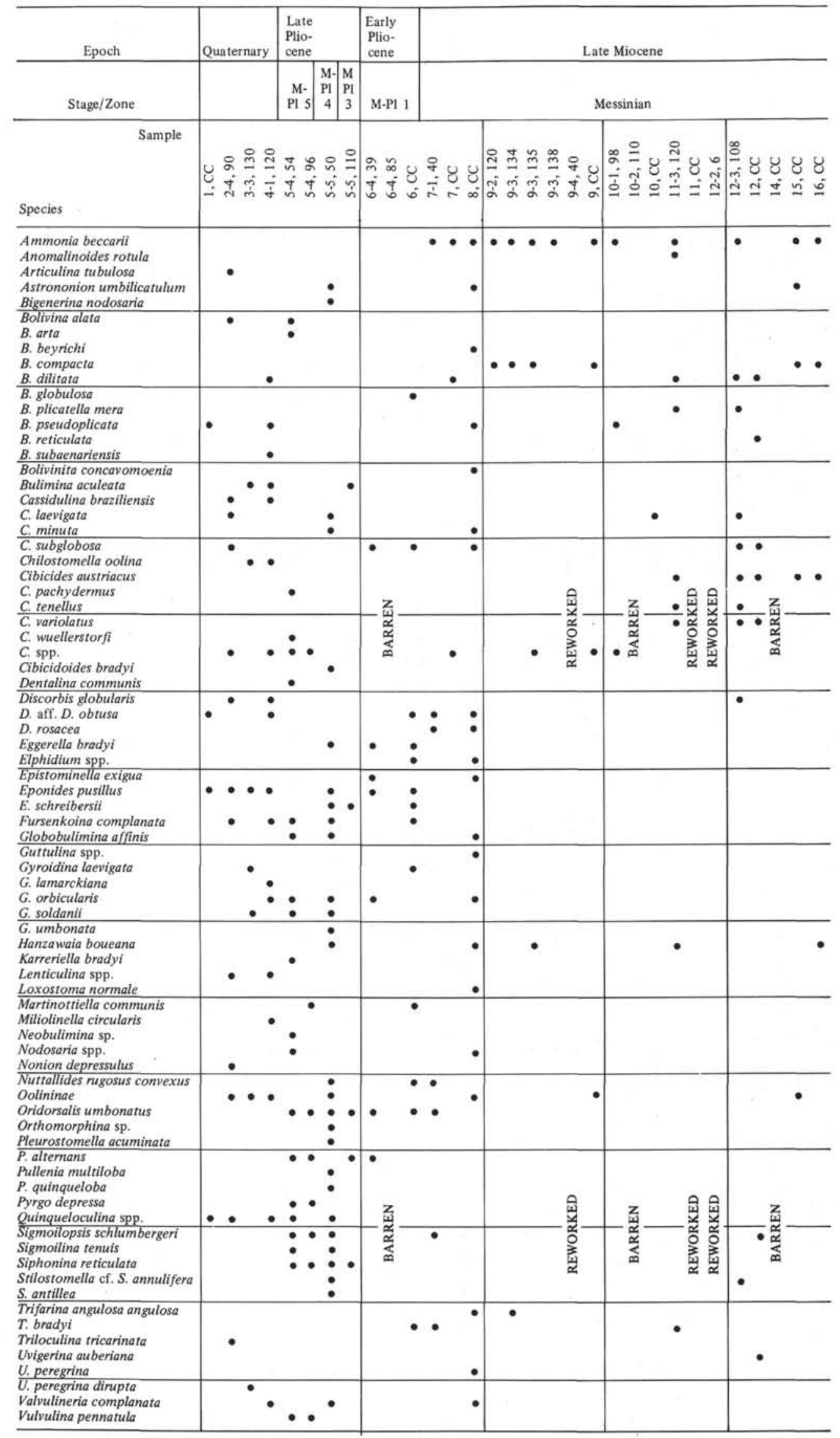


TABLE 31

Biostratigraphic Distribution of Benthic Foraminifers at Site 377

\begin{tabular}{|c|c|c|}
\hline Epoch & Quat & $\begin{array}{c}\text { E./M. } \\
\text { Miocene }\end{array}$ \\
\hline Species & 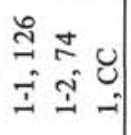 & 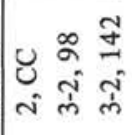 \\
\hline $\begin{array}{l}\text { Ammonia beccarii } \\
\text { Anomalina cicatricosa } \\
\text { Articulina tubulosa } \\
\text { Bolivina catanensis } \\
\text { B. globulosa }\end{array}$ & $\begin{array}{lll}\bullet & \bullet \\
& \bullet & \bullet \\
\bullet & \end{array}$ & 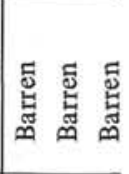 \\
\hline $\begin{array}{l}\text { Cassidulina laevigata } \\
\text { Eponides pusillus } \\
\text { Gyroidina soldanii } \\
\text { Oolinae } \\
\text { Pleurostomella alternans }\end{array}$ & \begin{tabular}{ll|}
$\bullet$ & $\bullet$ \\
$\bullet$ & $\bullet$ \\
$\bullet$ &
\end{tabular} & \\
\hline $\begin{array}{l}\text { Pullenia quinqueloba } \\
\text { P. salisburyi } \\
\text { Quinqueloculina spp. }\end{array}$ & • & \\
\hline
\end{tabular}


TABLE 32

Biostratigraphic Distribution of Benthic Foraminifers at Holes 378 and 378A

\begin{tabular}{|c|c|c|c|c|c|c|c|}
\hline Epoch & Quaternary & Late $\mathrm{F}$ & Pliocene & e 1 & Early I & Plioc & cene \\
\hline Stage/Zone & & $\begin{array}{r}\text { M- } \\
\text { Pl } 6\end{array}$ & $\begin{array}{l}\text { M- } \\
\text { P1 } 5\end{array}$ & & \begin{tabular}{l|l} 
M- & 1 \\
14 & $P$
\end{tabular} & $\begin{array}{l}\text { M- } \\
\text { P1 } 3\end{array}$ & $\begin{array}{l}\text { M- } \\
\text { Pl } 2\end{array}$ \\
\hline Species & 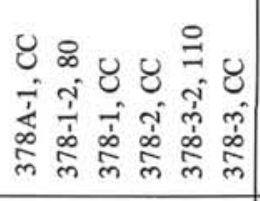 & $\begin{array}{ll}0 & 0 \\
0 & 8 \\
n & 0 \\
0 & 0 \\
0 & 0 \\
0 & 0 \\
n & 0\end{array}$ & 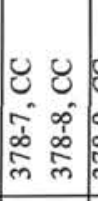 & $\begin{array}{l}0 \\
0 \\
0 \\
0 \\
0 \\
0 \\
m\end{array}$ & 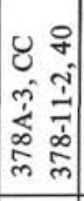 & 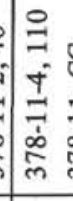 & 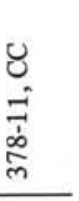 \\
\hline $\begin{array}{l}\text { Articulina tubulosa } \\
\text { Astrononion umbilicatulum } \\
\text { Bigenerina nodosaria } \\
\text { Bolivina alata } \\
\text { B. albatrossi } \\
\end{array}$ & $\begin{array}{llll}\bullet & \bullet & \bullet & \bullet \\
\bullet & & & \\
\bullet & \bullet & \bullet\end{array}$ & & & & & $\bullet$ & $\bullet$ \\
\hline $\begin{array}{l}\text { B. arta } \\
\text { B. catanensis } \\
\text { B. compacta } \\
\text { B. dilitata } \\
\text { B. pseudoplicata }\end{array}$ & $\bullet$ & & $\bullet$ & & & & : \\
\hline $\begin{array}{l}\text { B. pseudopunctata } \\
\text { B. reticulata } \\
\text { B. spathulata } \\
\text { B. subspinescens } \\
\text { B. variablis } \\
\end{array}$ & $\bullet$ & & & & $\bullet \bullet$ & & $\begin{array}{l}\bullet \\
\bullet \\
\bullet\end{array}$ \\
\hline $\begin{array}{l}\text { Bulimina aculeata } \\
\text { B. alazanensis } \\
\text { B. inflata } \\
\text { B. marginata } \\
\text { Cassidulina crassa } \\
\end{array}$ & $\bullet$ & $\bullet$ & • & $\bullet$ & $\bullet$ & • & - \\
\hline $\begin{array}{l}\text { C. braziliensis } \\
\text { C. laevigata } \\
\text { C. minuta } \\
\text { C. subglobosa } \\
\text { Chilostomella oolina } \\
\end{array}$ & $\begin{array}{llll}\bullet & \bullet & \bullet & \bullet \\
\bullet & \bullet & \bullet & \bullet \\
& & & \bullet\end{array}$ & \begin{tabular}{rl|}
$\bullet$ & $\bullet$ \\
$\bullet$ & $\bullet$
\end{tabular} & $\begin{array}{ll}\bullet & \bullet \\
\bullet & \bullet\end{array}$ & $\bullet$ & $\bullet \bullet$ & & • \\
\hline $\begin{array}{l}\text { Cibicides austriacus } \\
\text { C. lobatulus } \\
\text { C. pachydermus } \\
\text { C. variolatus } \\
\text { C. spp. } \\
\end{array}$ & - $\quad \bullet$ & & $\bullet \bullet$ & $\bullet$ & $\bullet$ & $\bullet$ & $\begin{array}{l}\bullet \\
\bullet \\
\bullet \\
\bullet\end{array}$ \\
\hline $\begin{array}{l}\text { Cibicidoides kullenbergi } \\
\text { Dentalina communis } \\
\text { Discorbis globularis } \\
\text { D. parkerae } \\
\text { D. rosacea } \\
\end{array}$ & $\bullet$ & • & $\bullet$ & $\bullet$ & $\bullet$ & $\bullet$ & $\begin{array}{l}\bullet \\
\bullet \\
\bullet\end{array}$ \\
\hline $\begin{array}{l}\text { Eggerella bradyi } \\
\text { Elphidium spp. } \\
\text { Epistominella exigua } \\
\text { Eponides pusillus } \\
\text { E. schreibersii } \\
\end{array}$ & • & $\bullet$ & • & & $\bullet$ & • & \\
\hline $\begin{array}{l}\text { Fursenkoina complanata } \\
\text { F. spp. } \\
\text { Gavelinopsis praegeri }\end{array}$ & • & & $\bullet$ & & & : & \\
\hline
\end{tabular}


TABLE 32 - Continued

\begin{tabular}{|c|c|c|c|c|c|c|c|}
\hline Epoch & Quaternary & Late & Pliocen & & Early & Plio & cene \\
\hline Stage/Zone & & $\begin{array}{l}\text { M- } \\
\text { Pl } 6\end{array}$ & $\begin{array}{l}\text { M- } \\
\text { Pl } 5\end{array}$ & & \begin{tabular}{l|l} 
M- & \\
14 & $\mathrm{P}$
\end{tabular} & $\begin{array}{l}\text { M- } \\
\text { PI } 3\end{array}$ & $\begin{array}{l}\text { M- } \\
\text { Pl } 2\end{array}$ \\
\hline Species & 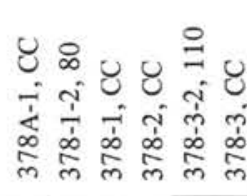 & \begin{tabular}{ll|}
$u$ & 0 \\
0 & 0 \\
0 & 0 \\
0 & 0 \\
0 & 0 \\
$m$ & $m$
\end{tabular} & 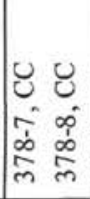 & - & 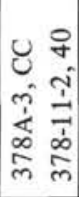 & 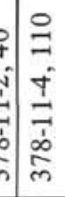 & 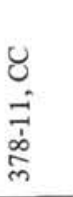 \\
\hline $\begin{array}{l}\text { Glabratella pulvinata } \\
\text { Gyroidina altiformis }\end{array}$ & - & & & & & & $\bullet$ \\
\hline $\begin{array}{l}\text { G. laevigata } \\
\text { G. orbicularis } \\
\text { Hanzawaia boueana } \\
\text { Hoeglundina elegans } \\
\text { Hyalinea balthica } \\
\end{array}$ & 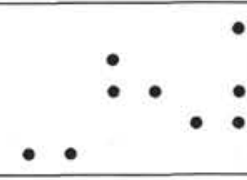 & - & • & & - & $\bullet$ & - \\
\hline $\begin{array}{l}\text { Karreriella bradyi } \\
\text { Lenticulina spp. } \\
\text { Melonis barleeanum } \\
\text { M. soldanii } \\
\text { Neobulimina sp. } \\
\end{array}$ & - $\bullet$ & $\bullet \bullet$ & - & $\bullet$ & $\bullet$ & $\bullet$ & $\bullet$ \\
\hline $\begin{array}{l}\text { Necconorbina terquemi } \\
\text { Nodosaria spp. } \\
\text { Nonion depressulus } \\
\text { N. scaphum } \\
\text { Nonionella } \text { sp. A }\end{array}$ & • & & $\because$ & & $\bullet$ & & $\bullet$ \\
\hline $\begin{array}{l}\text { Oolininae } \\
\text { Oridorsalis umbonatus } \\
\text { Planulina ariminensis } \\
\text { Pleurostomella alternans } \\
P \text {. spp. }\end{array}$ & • $\bullet$ & \begin{tabular}{rr|} 
& $\bullet$ \\
& $\bullet$ \\
& $\bullet$
\end{tabular} & $\begin{array}{l}\bullet \\
\bullet\end{array}$ & $\bullet$ & & - & : \\
\hline $\begin{array}{l}\text { Pseudoclavulina crustata } \\
\text { Pullenia bulloides } \\
\text { P. quinqueloba } \\
\text { Quinqueloculina venusta } \\
\text { Q. spp. }\end{array}$ & $\bullet \bullet \bullet$ & 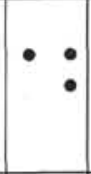 & : & $\bullet$ & $\bullet$ & $\bullet$ & : \\
\hline $\begin{array}{l}\text { Robertina translucens } \\
\text { Seabrookia earlandi } \\
\text { Sigmoilina tenuis } \\
\text { Sigmoilopsis schlumbergeri } \\
\text { Siphonina reticulata }\end{array}$ & $\begin{array}{llll} & & & \bullet \\
\bullet & \bullet & \bullet & \bullet \\
& & \bullet\end{array}$ & $\bullet$ & • $\bullet$ & $\bullet$ & & • & \\
\hline $\begin{array}{l}\text { S. tubulosa } \\
\text { Sphaeroidina bulloides } \\
\text { Trifarina angulosa angulosa } \\
\text { T. angulosa pauperata } \\
\text { T. bradyi }\end{array}$ & • $\bullet$ & - & - $\bullet$ & $\bullet$ & $\begin{array}{r} \\
\bullet \\
\end{array}$ & - : & - \\
\hline $\begin{array}{l}\text { Triloculina tricarinata } \\
\text { Uvigerina longistriata } \\
U \text {. mediterranea } \\
U \text {. mioindex } \\
\text { U. peregrina } \\
\end{array}$ & - & $\bullet$ & $\bullet \bullet$ & $\bullet$ & & & : \\
\hline $\begin{array}{l}U \text {. peregrina dirupta } \\
U \text {. rutila }\end{array}$ & - $\bullet$ & & & & - $\bullet$ & - $\bullet$ & $\bullet$ \\
\hline
\end{tabular}

